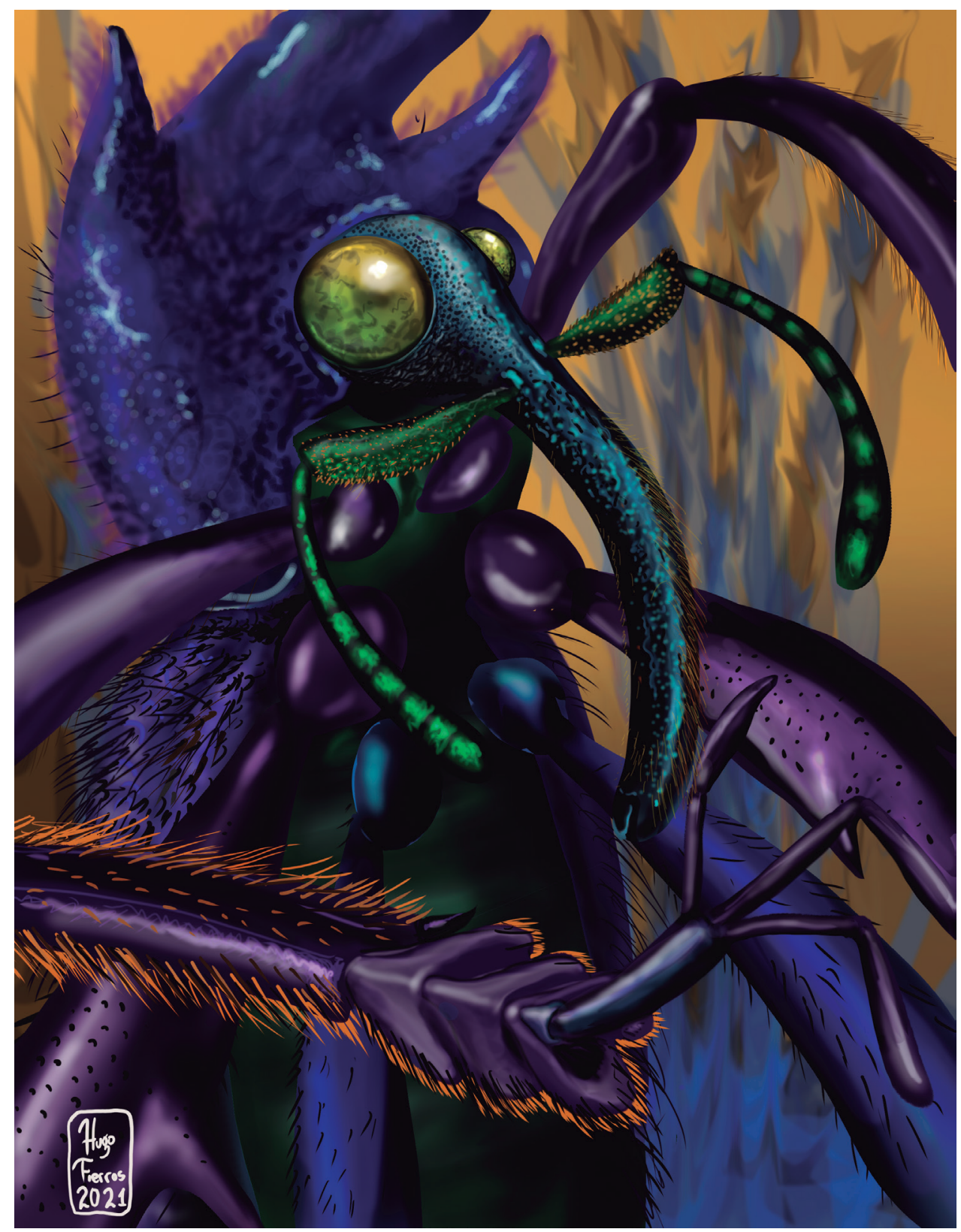

Dugesiana, Año 28, No. 2, (julio-diciembre, segundo semestre de 2021), es una publicación semestral, editada por la Universidad de Guadalajara, a través del Centro de Estudios en Zoología, por el Centro Universitario de Ciencias Biológicas y Agropecuarias. Camino Ramón Padilla Sánchez \# 2100, Nextipac, Zapopan, Jalisco, Tel. 37771150 ext. 33218, http://148.202.248.171/dugesiana/index.php/DUG/index, glenusmx@gmail.com. Editor responsable: José Luis Navarrete-Heredia. Reserva de Derechos al Uso Exclusivo 04-2009-062310115100-203, ISSN: 2007-9133, otorgados por el Instituto Nacional del Derecho de Autor. Responsable de la última actualización de este número: José Luis Navarrete-Heredia, Editor y Ana Laura González-Hernández, Asistente Editorial. Fecha de la última modificación 1 de julio de 2021, con un tiraje de un ejemplar.

Las opiniones expresadas por los autores no necesariamente reflejan la postura del editor de la publicación.

Queda estrictamente prohibida la reproducción total o parcial de los contenidos e imágenes de la publicación sin previa autorización de la Universidad de Guadalajara. 


\title{
El género Onthophagus Latreille, 1802 (Coleoptera: Scarabaeidae) de México
}

\author{
The genus Onthophagus Latreille, 1802 (Coleoptera: Scarabaeidae) of Mexico
}

\author{
Victor Moctezuma \\ Investigador independiente, Puebla, Puebla, MÉXICO abadonjvpm@hotmail.com, \\ ORCID: https://orcid.org/0000-0002-4532-0302
}

\begin{abstract}
RESUMEN
Se utilizó información proveniente de literatura para compilar el listado de la fauna de Onthophagus de México. Se encontraron un total de 120 especies, de las cuales 85 son endémicas de México. Se incluyen los patrones de distribución originalmente propuestos por Halffter (1964) y comentarios adicionales para la mayoría de las especies. Se encontraron registros de especies para todos los estados de la República Mexicana, con excepción de Baja California. México es el centro de diversificación de Onthophagus de América, con aproximadamente el $62 \%$ de las especies descritas para el continente. Oaxaca es el estado con mayor riqueza de especies y mayor número de endemismos. Se discuten los factores que promovieron la diversificación de Onthophagus en México. Onthophagus mesoamericanus Zunino y Halffter, 1988 es resucitado de la sinonimia previa.

Palabras clave: Scarabaeinae, Onthophagini, escarabajos del estiércol, Zona de Transición Mexicana, Paleoamericano, Boreotropical, endemismo, montañas.
\end{abstract}

Data from literature was gathered to compile the checklist of the fauna of Onthophagus of Mexico. A total amount of 120 species was found, with 85 endemics to Mexico. The distributional pattern as originally proposed by Halffter (1964) and commentaries of the majority of the species are provided. Records of species were found for all the Mexican States, except for Baja California. Mexico is the diversification center of Onthophagus in America, with ca. $62 \%$ of the described species of the continent. Oaxaca is the richest state and the only one with the greater number of endemisms. The factors that drove the diversification of Onthophagus in Mexico are discussed. Onthophagus mesoamericanus Zunino y Halffter, 1988 is resurrected from previous synonymy.

Key words: Scarabaeinae, Onthophagini, dung beetles, Mexican Transition Zone, Paleoamerican, Boreotropical, endemism, mountains.

Onthophagus Latreille, 1802 es un grupo de organismos realmente notable, pues al estar integrado por más de 2200 especies es indudablemente uno de los géneros de animales más diversos del Mundo (Howden y Cartwright 1963; Breeschoten et al. 2016; Schoolmeesters 2021). Onthophagus es considerado como un género cuasi cosmopolita, estando ausente únicamente en la Antártida, y pudiéndose encontrar en todos los ecosistemas desde el nivel del mar hasta los 5000 m.s.n.m.; siendo típicamente moradores del suelo, frecuentando ocasionalmente el dosel, o incluso siendo habitantes de madrigueras y cuevas (Cambefort 1991; Pulido-Herrera y Zunino 2007; Zunino y Halffter 2007; Moctezuma y Halffter 2021). Los Onthophagus son usualmente reconocidos por sus hábitos coprófagos, aunque numerosas especies pueden recurrir a la necrofagia, saprofagia o micofagia (Halffter y Matthews 1966; Anduaga 2000; Halffter y Halffter 2009; Moretto 2010; Moctezuma et al. 2016a; Joaqui et al. 2019), por lo que proveen servicios ambientales clave a los ecosistemas naturales y antropizados, y son ampliamente recomendados como indicadores biológicos (Nichols et al. 2007; 2008; Lumaret et al. 2020).

Se ha reconocido que el género Onthophagus no es un grupo monofilético en su actual conformación, pero las especies presentes en el continente americano sí comparten un ancestro en común (Breeschoten et al. 2016; Philips 2016;
Tarasov y Dimitrov 2016; Schwery y O’Meara 2021), lo que contradice la idea de que las especies americanas de Onthophagus se originaron a partir de al menos 20 ancestros distintos (Howden y Gill 1993). El género Onthophagus tiene una presencia notable en el continente americano donde se localizan más de 190 especies (Moctezuma y Halffter 2021), que actualmente se distribuyen desde el extremo sur de Canadá hasta la región central de Argentina (Howden y Cartwright 1963; Zunino y Halffter 1997; Rossini et al. 2018a; Moctezuma y Halffter 2021), aunque existe evidencia que sugiere que el género se encontraba hasta el sur de Chile en periodos geológicos anteriores (Tello et al. 2021). Las especies americanas de Onthophagus han sido clasificadas tradicionalmente en distintos grupos de especies (Zunino y Halffter 1988a; 1997; Howden y Gill, 1993; Kohlmann y Solís, 2001). Sin embargo, estudios filogenéticos recientes apoyan la idea de que los grupos de especies de Onthophagus de América son grupos no naturales (Moctezuma et al. 2019; Schwery y O’Meara 2021).

El estudio de los Onthophagus del continente americano no ha sido uniforme, pues un número limitado de países se ha reconocido por concentrar los mayores esfuerzos para el conocimiento de su fauna. De esta forma, Canadá (Howden y Cartwright 1963), Estados Unidos (Howden y Cartwright 1963; Zunino y Halffter 1988a), México (Zunino y Halffter 
1988a; Zunino 2003), Costa Rica (Kohlmann y Solís 2001) y Panamá (Howden y Young 1981; Howden y Gill 1987; Delgado y Curoe 2014) destacan por ser los mejor estudiados con respecto a su fauna de Onthophagus. Además, los estudios sobre el género Onthophagus no suelen ser muy frecuentes y existen pocos especialistas capacitados para su determinación taxonómica. En consecuencia, el presente trabajo se centrará particularmente en el estudio de los Onthophagus de México.

El primer intento moderno de sintetizar la fauna de $\mathrm{On}$ thophagus de México fue hecho por Zunino (2003), que fue ampliado por la contribución de Pulido-Herrera y Zunino (2007) sobre los Onthophagus de América. Además, los Onthophagus de México han recibido particular atención en años recientes, lo que se ha visto reflejado en numerosos cambios taxonómicos y en la descripción de numerosas especies nuevas. Sin embargo, las recientes aportaciones al conocimiento de los Onthophagus de México suelen ser parte de estudios dispersos y fragmentarios (Rossini et al. 2018a). En consecuencia, el objetivo del presente estudio es actualizar el listado de especies de México; así como sintetizar los numerosos cambios taxonómicos y nomenclaturales que han surgido recientemente en la literatura sobre este género.

\section{MATERIAL Y MÉTODOS}

Para compilar el listado actualizado de Onthophagus de México fueron utilizadas como línea base las aportaciones de Zunino (2003) y Pulido-Herrera y Zunino (2007), además de que se realizó una búsqueda extensiva de literatura que contiene información relevante sobre las especies distribuidas en el país, las cuales serán citadas. Como se mencionó en la sección introductoria, Onthophagus ha sido incluido tradicionalmente en distintos grupos de especies. Aunque se reconoce la utilidad de los grupos de especies para facilitar la determinación taxonómica de Onthophagus, estos grupos no representan unidades evolutivas monofiléticas (Moctezuma et al. 2019; Schwery y O’Meara 2021), por lo que el presente listado seguirá un orden alfabético y los grupos de especies serán omitidos. Es probable que la clasificación infragenérica actual de los Onthophagus de América sea abruptamente modificada o que caiga en desuso en años próximos, tal como ocurrió con los grupos propuestos por Boucomont (1932).

Además de las especies nativas presentes en México, se incluyen los distintos sinónimos, subespecies, registros erróneos o dudosos, algunos nombres no disponibles, y la distribución conocida de cada especie. Para referir a los estados que integran a la República Mexicana, se utilizaron las siguientes abreviaturas: Aguascalientes (AGS), Baja California Sur (BJS), Ciudad de México (CDMX), Campeche (CAM), Chiapas (CHIS), Chihuahua (CHIH), Coahuila (COA), Colima (COL), Durango (DGO), Estado de México (EDOMEX), Guerrero (GRO), Guanajuato (GTO), Hidalgo (HGO), Jalisco (JAL), Michoacán (MICH), Morelos (MOR), Nayarit (NAY), Nuevo León (NL), Oaxaca (OAX), Puebla (PUE), Quintana Roo (QR), Querétaro (QRO), Sinaloa (SIN), Sonora (SON), San Luis Potosí (SLP), Tabasco (TAB), Tamaulipas (TAM), Tlaxcala (TLA), Veracruz
(VER), Yucatán (YUC), Zacatecas (ZAC).

También es importante mencionar que dentro de la composición del listado se va hacer referencia al patrón de distribución que corresponde con cada especie, tomando en consideración el criterio propuesto originalmente por Halffter (1964), que ha sido sintetizado y ampliado por numerosos estudios posteriores (Zunino y Halffter 1988; Halffter et al. 1995; 2008; 2012; 2019; Halffter 2017; 2019; Halffter y Morrone 2017; Moctezuma y Halffter 2019a; Morrone 2020).

\section{RESULTADOS}

\section{Listado de especies de Onthophagus de México}

Onthophagus Latreille, 1802: 20

1. O. acuminatus Harold, 1880

CHIS, OAX, VER; Nicaragua, Costa Rica, Panamá, Colombia, Ecuador, Perú.

Onthophagus acuminatus Harold, 1880: 30; Boucomont y Gillet 1927; Boucomont 1932; Paulian 1933; 1936; Blackwelder 1944; Pereira y Halffter 1961; Halffter y Matthews 1966; Vulcano y Pereira 1967; Howden y Young 1981; Gill 1991; Escobar y Medina 1996; Delgado 1997; Emlen 1997; Hunt y Simmons 1997; Zunino y Halffter 1997; Nijhout y Emlen 1998; Emlen y Nijhout 2000; Escobar 2000; 2004; Escobar y Chacón de Ulloa 2000; Moczek y Emlen 2000; Barbero 2001; Kohlmann y Solís 2001; Medina et al. 2001; Hernández et al. 2003; Sansom 2003; Zunino 2003; Krajcik 2006; Kohlmann et al. 2007; Noriega et al. 2007; Padilla-Hill y Halffter 2007; Pulido-Herrera y Zunino 2007; Escobar et al. 2008; Navarrete y Halffter 2008; Halffter y Halffter 2009; Martínez et al. 2009; Medina y Pulido 2009; Granados et al. 2010; Carvajal et al. 2011; Deloya-López 2011; Knell 2011; Philips 2011; Solís et al. 2011; Tarasov y Solodovnikov 2011; Cultid-Medina et al. 2012; Pereira y Kohlmann 2012; Solís y Kohlmann 2012; Arellano et al. 2013; Delgado y Curoe 2014; Medina y González 2014; González-Alvarado y Medina 2015; González-Alvarado et al. 2015; Ratcliffe et al. 2015; Sánchez-de-Jesús et al. 2016; Chamorro et al. 2018; 2019; Rossini et al. 2018a; Amell-Caez et al. 2019; Ramírez-Ponce et al. 2019; Sánchez-Huerta et al. 2019; Sánchez-Hernández et al. 2020; Moctezuma 2021; Noriega et al. 2021; Schwery y O'Meara 2021; Whitworth et al. 2021.

Patrón de distribución: Paleoamericano tropical.

Comentarios: Es necesario actualizar la distribución de esta especie, ya que actualmente se encuentra en revisión (Rossini et al. 2018a; 2018b).

2. O. alluvius Howden y Cartwright, 1963

HGO, NL, OAX, PUE; QRO, SLP, TAM; Estados Unidos.

Onthophagus alluvius Howden y Cartwright, 1963: 65; Halffter y Matthews 1966; Edmonds y Halffter 1978; Delgado 1997; Zunino y Halffter 1997; 
Zunino 2003; Delgado y Montes de Oca 2005; García-Morales y Montes de Oca 2005; Krajcik 2006; Pulido-Herrera y Zunino 2007; Halffter et al. 2008; 2012; Martínez-H et al. 2010; Barragán et al. 2011; Ramírez-Restrepo y Halffter 2016; Márquez et al. 2017; Pinilla-Buitrago et al. 2018; Arriaga-Jiménez et al. 2019; Bezanson y Floate 2019.

Patrón de distribución: Paleoamericano del Altiplano Mexicano.

Comentarios: Esta especie requiere de revisión taxonómica, ya que es posible que se trate de un complejo de especies crípticas.

3. O. altivagans Howden y Génier, 2004

DGO, SON; Estados Unidos.

Onthophagus altivagans Howden y Génier, 2004: 71; Pulido-Herrera y Zunino 2007; Arriaga-Jiménez et al. 2019.

Patrón de distribución: Paleoamericano del Altiplano Mexicano.

4. O. anewtoni Howden y Génier, 2004

CHIS, GRO, JAL, ¿MOR? OAX, ¿PUE?

Onthophagus anewtoni Howden y Génier, 2004: 55; Pulido-Herrera y Zunino 2007; Deloya y Covarrubias-Melgar 2014; Sánchez-Hernández y Gómez 2018; Moctezuma y Halffter 2021.

Patrón de distribución: Paleoamericano del Altiplano Mexicano.

Comentarios: Esta especie probablemente ha sido citada como O. championi en los estados de Puebla y Morelos (Moctezuma y Halffter 2021).

5. O. anthracinus Harold, 1873

CHIS, OAX, PUE, VER; Guatemala, Belice, Honduras, Costa Rica, Panamá.

Onthophagus anthracinus Harold, 1873: 104; Boucomont 1932; Blackwelder 1944; Halffter y Matthews 1966; Deloya 1992b; Thomas 1993; Zunino y Halffter 1997; Kohlmann y Solís 2001; Zunino 2003; Krajcik 2006; Kohlmann et al. 2007; 2018; 2020; Pulido-Herrera y Zunino 2007; Creedy y Mann 2011; Deloya-López 2011; Inward et al. 2011; Delgado et al. 2012b; Solís y Kohlmann 2012; Sánchez-Hernández et al. 2017; 2020; Pinilla-Buitrago et al. 2018; Arriaga-Jiménez et al. 2019; Rodríguez-López et al. 2019; Moctezuma 2021.

Patrón de distribución: Paleoamericano de montaña.

Comentarios: Arriaga-Jiménez et al. (2019) erróneamente sugieren que presentaron registros nuevos de país para esta especie en Guatemala: "The following specimens represent country records for Guatemala". Arriaga-Jiménez et al. (2019) omitieron el hecho de que Harold (1873) describió originalmente a esta especie de Guatemala, mientras que Kohlmann y Solís (2001) y Pulido-Herrera y Zunino (2007) también citaron a $O$. anthracinus en ese país.

6. O. asperodorsatus Howden y Gill, 1993 VER.
Onthophagus asperodorsatus Howden y Gill, 1993: 1095; Navarrete-Heredia y Galindo-Miranda 1997; Zunino y Halffter 1997; Zunino 2003; Krajcik 2006; Pulido-Herrera y Zunino 2007; Deloya-López 2011; Génier 2017; Delgado y Mora-Aguilar 2019; Moctezuma y Halffter 2019b; Salomão et al. 2020.

Patrón de distribución: Paleoamericano tropical.

7. O. atriglabrus Howden y Gill, 1987

CHIS; Costa Rica, Panamá, Colombia.

Onthophagus atriglabrus Howden y Gill, 1987: 218; Delgado y Deloya 1990; Gill 1991; Escobar y Medina 1996; Delgado 1995; Zunino y Halffter 1997; Escobar 2000; Kohlmann y Solís 2001; Medina et al. 2001; Neita et al. 2003; Krajcik 2006; Kohlmann et al. 2007; Pulido-Herrera y Zunino 2007; Halffter y Halffter 2009; Solís y Kohlmann 2012; Delgado y Curoe 2014; Sánchez-Hernández y Gómez 2018.

Patrón de distribución: Paleoamericano tropical.

8. O. aureofuscus Bates, 1887 HGO, OAX, PUE, TLA, VER.

Onthophagus aureofuscus Bates, 1887: 81; Boucomont y Gillet 1927; Boucomont 1932; Blackwelder 1944; Zunino y Halffter 1988a; Deloya 1992b; Martín-Piera y Lobo 1993; Halffter et al. 1995; 2008; 2019; Lobo y Halffter 2000; Zunino 2003; Krajcik 2006; Escobar et al. 2007; Pulido-Herrera y Zunino 2007; Ramírez-Ponce et al. 2009; Aragón-García et al. 2011; Deloya-López 2011; Morón et al. 2013; Moctezuma et al. 2016b; Márquez et al. 2017; Arriaga-Jiménez et al. 2018; Kohlmann et al. 2018; Pinilla-Buitrago et al. 2018; Escobar-Hernández et al. 2019; Alvarado et al. 2020; Moctezuma y Halffter 2020a; Salomão et al. 2021.

Patrón de distribución: Paleoamericano de montaña. Comentarios: Deloya-López (2011) utilizó la grafía posterior incorrecta "aerofuscus" para referirse a esta especie. Autores anteriores han sugerido que esta especie se encuentra en peligro de extinción o casi amenazada de acuerdo con los criterios de la Lista Roja de Especies Amenazadas de la Unión Internacional para la Conservación de la Naturaleza (ver detalles en Escobar-Hernández et al. 2019). Sin embargo, no hay evidencia para considerar que $O$. aureofuscus está próximo a satisfacer los requisitos para estar incluida en tales categorías de riesgo. Para empezar, O. aureofuscus es una especie de amplia distribución en la región central de México, con una extensión de ocupación mayor a 30,000 $\mathrm{km}^{2}$ (ver distribución en Moctezuma y Halffter 2020a). Por otra parte, $O$. aureofuscus puede considerarse como una especie relativamente abundante (Martín-Piera y Lobo 1993; Ramírez-Ponce et al. 2009; Moctezuma et al. 2016a; 2016b; Arriaga-Jiménez et al. 2018; Escobar-Hernández et al. 2019; Salomão et al. 2021), que incluso llega a presentar una 
dominancia completa del ensamble de Scarabaeinae presente en algunas montañas del centro de México (Moctezuma et al. 2016a; 2016b). Finalmente, es posible que la abundancia de esta especie se incremente en algunos ambientes de montaña que han sido perturbados por actividades antropogénicas de moderada intensidad (Moctezuma et al. 2016a; 2016b). Por lo tanto, esta especie debería estar considerada dentro de la categoría de preocupación menor.

9. O. aztecus Zunino y Halffter, 1988

CDMX, MOR.

Onthophagus aztecus Zunino y Halffter, 1988a: 157; Zunino 2003; Krajcik 2006; Pulido-Herrera y Zunino 2007; Arriaga-Jiménez et al. 2016; Halffter et al. 2019; Zaragoza-Caballero et al. 2019.

Patrón de distribución: Paleoamericano de montaña.

Comentarios: Los registros de O. aztecus en Puebla (Aragón-García et al. 2011; Morón et al. 2013) corresponden a $O$. bolivari.

10. O. bajacalifornianus Moctezuma y Halffter, 2019 BCS.

Onthophagus bajacalifornianus Moctezuma y Halffter, 2019a: 2060 (Figura 1); Morrone 2020.

Patrón de distribución: Paleoamericano bajacaliforniano.

11. O. bassariscus Zunino y Halffter, 1988 GRO.

Onthophagus bassariscus Zunino y Halffter, 1988a: 60; 2007; Halffter y Matthews 1966 (citado como especie nueva sin describir); Delgado 1999; Zunino 2003; Krajcik 2006; Pulido-Herrera y Zunino 2007; Deloya y Covarrubias-Melgar 2014; Sánchez-Huerta et al. 2015; 2018; Halffter 2017; 2019; Halffter y Morrone 2017; Gasca-Álvarez et al. 2018; Halffter et al. 2019; Morrone 2020.

Patrón de distribución: Paleoamericano mesoamericano.

12. O. batesi Howden y Cartwright, 1963

CAM, CHIS, COL, GRO, HGO, JAL, MICH, MOR, OAX, PUE, SLP, TAB, TAM, VER, YUC; Estados Unidos, Belice, El Salvador, Nicaragua, Costa Rica, Panamá, Antillas, Colombia.

Onthophagus batesi Howden y Cartwright, 1963: 21; Halffter y Matthews 1966; Matthews 1966; Morón 1979; 1985; 1987; Edmonds y Halffter 1978; Howden y Young 1981; Morón et al. 1985; 2013; Gill 1991; Deloya 1992b; Halffter et al. 1992; 2008; Estrada et al. 1993; 1998; Thomas 1993; Montes de Oca y Halffter 1995; Delgado 1997; Morón y Blackaller 1997; Zunino y Halffter 1997; Maes 1998; Martínez y Cruz 1999; Palacios-Ríos et al. 1990; Emlen y Nijhout 2000; Escobar 2000; Halffter y Arellano 2002; Kohlmann y Solís 2001; Montes de Oca 2001; Navarrete-Heredia et al. 2001; Medina et al. 2001; Estrada y Coates-Estrada 2002; Carrillo-Ruíz y Morón 2003; Martínez y Lumaret 2003; Zunino 2003; Delgado y Montes de Oca
2005; García-Morales y Montes de Oca 2005; Krajcik 2006; Escobar et al. 2007; 2008; Esparza-León y Amat-García 2007; Horgan 2007; 2008; Kohlmann et al. 2007; Monaghan et al. 2007; Padilla-Hill y Halffter 2007; Pulido-Herrera y Zunino 2007; Reyes-Novelo et al. 2007; Arellano et al. 2008; 2013; Navarrete y Halffter 2008; Halffter y Halffter 2009; Mora-Aguilar y Montes de Oca 2009; Ramírez-Ponce et al. 2009; Díaz et al. 2010; 2011; Granados et al. 2010; Barragán et al. 2011; Deloya-López 2011; Inward et al. 2011; Martínez et al. 2011; 2017; Delgado et al. 2012a; 2012b; Favila 2012; Pereira y Kohlmann 2012; Solís y Kohlmann 2012; Alvarado et al. 2014; 2018a; 2018b; 2020; Delgado y Curoe 2014; Deloya y Covarrubias-Melgar 2014; Morales-Morales et al. 2014; Zamora-Vuelvas et al. 2014; González-Hernández et al. 2015; Deloya et al. 2016; Gunter et al. 2016; Morón y Arce-Pérez 2016; Sánchez-de-Jesús et al. 2016; Márquez et al. 2017; González-Tokman et al. 2018; Pinilla-Buitrago et al. 2018; Salomão et al. 2018; Capello y Halffter 2019; Rodríguez-López et al. 2019; Santos-Heredia et al. 2019; Velázquez-López et al. 2019; Zaragoza-Caballero et al. 2019; Miranda-Flores et al. 2020; Pablo-Cea et al. 2020; Rivera et al. 2020; Rivera-Gasperín y Escobar-Hernández 2020; Salomão et al. 2020; Sánchez-Hernández et al. 2020; Moctezuma 2021; Whitworth et al. 2021.

Patrón de distribución: Paleoamericano tropical.

Comentarios: Es necesario actualizar la distribución de esta especie, ya que actualmente se encuentra en revisión (Rossini et al. 2018a; 2018b). García-Morales y Montes de Oca (2005) utilizaron la grafía posterior incorrecta "batesii", mientras que Estrada et al. (1998) utilizaron la grafía posterior incorrecta "bates" para referirse a esta especie.

13. O. belorhinus Bates, 1887

CHIS, HGO, OAX, PUE, VER; Guatemala, Nicaragua, El Salvador, Panamá, Colombia, Ecuador.

Onthophagus belorhinus Bates, 1887: 69; Boucomont y Gillet 1927; Boucomont 1932; Blackwelder 1944; Halffter y Matthews 1966; Pereira y Halffter 1961; Howden y Young 1981; Morón et al. 1986; 2013; Morón 1987; Delgado y Deloya 1990; Gill 1991; Thomas 1993; Navarrete-Heredia y Galindo-Miranda 1997; Zunino y Halffter 1997; Delgado 1995; Delgado y Pensado 1998; Maes 1998; Escobar 2000; Medina et al. 2001; Anduaga 2000; Carrillo-Ruíz y Morón 2003; Zunino 2003; Krajcik 2006; Delgado et al. 2006; Deloya et al. 2007; Esparza-León y Amat-García 2007; Pulido-Herrera y Zunino 2007; Halffter y Halffter 2009; Villamarín-Cortez 2010; Deloya-López 2011; Rös et al. 2012; Alvarado et al. 2014; 2020; Barragán et al. 2014; Huerta-Crespo y Cruz-Rosales 2016; Huerta et al. 2016; Már- 
quez et al. 2017; Rossini et al. 2018a; Arellano et al. 2020; Moctezuma et al. 2020; Pablo-Cea et al. 2020; Sánchez-Hernández et al. 2020; Schwery y O'Meara 2021.

Patrón de distribución: Paleoamericano tropical.

Comentarios: Alvarado et al. (2014) utilizaron la grafía posterior incorrecta "belorhynus" para referirse a esta especie.

14. O. bolivari Moctezuma, Rossini y Zunino, 2016 PUE, TLA.

Onthophagus bolivari Arriaga-Jiménez et al., 2016: 136; 2018; Moctezuma et al. 2016a; 2016b; Halffter et al. 2019; Joaqui et al. 2019.

Patrón de distribución: Paleoamericano de montaña.

15. O. brachypterus Zunino y Halffter, 1997 COL, JAL.

Onthophagus brachypterus Zunino y Halffter, 1997: 169; Delgado y Howden 2000; Zunino 2003; Krajcik 2006; Pulido-Herrera y Zunino 2007; Gasca-Álvarez et al. 2018; Arriaga-Jiménez et al. 2019; Escobar-Hernández et al. 2019.

Patrón de distribución: Paleoamericano de montaña.

16. O. brevifrons Horn, 1881

DGO, SLP; Estados Unidos.

Onthophagus brevifrons Horn, 1881: 87; Henshaw 1885; Schaeffer 1914; Leng 1920; Boucomont y Gillet 1927; Boucomont 1932; Howden y Cartwright 1963; Halffter y Matthews 1966; Zunino 1980; Paulian 1988; Zunino y Halffter 1988a; 1988b; 2007; Anduaga y Halffter 1991; Cokendolpher y Polyak 1996; Delgado 1999; Zunino 2003; Morón 2004; Krajcik 2006; Slay et al. 2012; Arriaga-Jiménez et al. 2016; Edmonds 2018; Bezanson y Floate 2019; Halffter et al. 2019; Morrone 2020; Rivera-Gasperín et al. 2021.

Patrón de distribución: Paleoamericano del Altiplano Mexicano.

17. O. browni Howden y Cartwright, 1963

$\mathrm{CHIH}$ ¿COA?, DGO; Estados Unidos.

Onthophagus browni Howden y Cartwright, 1963: 101; Halffter y Matthews 1966; Delgado 1999; Anduaga y Halffter 1991; Zunino 2003; Edmonds 2004; 2018; Krajcik 2006; Anduaga 2007; Pulido-Herrera y Zunino 2007; Zunino y Halffter 2007; Moctezuma y Halffter 2019a; 2021.

Patrón de distribución: Paleoamericano del Altiplano Mexicano.

Comentarios: Edmonds (2004), sugiere que una especie cercana a $O$. browni se encuentra en Sonora, por lo que su registro requiere confirmación.

18. O. canelasensis Howden y Génier, 2004 DGO.

Onthophagus canelasensis Howden y Génier, 2004 73; Pulido-Herrera y Zunino 2007; Arriaga-Jiménez et al. 2019.

Patrón de distribución: Paleoamericano del Altiplano Mexicano.
19. O. canescens Zunino y Halffter, 1988 MICH, EDOMEX.

Onthophagus canescens Zunino y Halffter, 1988a: 83; Jiménez-Sánchez et al. 2009; Zamora-Vuelvas et al. 2014; Deloya et al. 2016; Moctezuma et al. 2016a; Zaragoza-Caballero y Pérez-Hernández 2017; Halffter et al. 2019; Joaqui et al. 2019; Moctezuma y Halffter 2020a; Morrone 2020.

Patrón de distribución: Paleoamericano de montaña.

Comentarios: Esta especie fue referida como $O$. fuscus y $O$. fuscus canescens por autores previos (ver Joaqui et al. 2019). La mayor parte del material típico de esta especie se encuentra perdida actualmente. En una búsqueda reciente en la colección personal del Dr. Gonzalo Halffter (2019), solamente fue posible encontrar 3 paratipos, mientras que la descripción original indica que en ella se encuentran al menos 16 machos y 14 hembras (Zunino y Halffter 1988a). El holotipo y un paratipo macho se encuentran depositados actualmente en la Colección Nacional de Insectos de la Universidad Nacional Autónoma de México (Zaragoza-Caballero y Pérez-Hernández 2017).

20. O. carpophilus Pereira y Halffter, 1961

CAM, CHIS, OAX, ¿VER?, YUC.

Onthophagus carpophilus Pereira y Halffter, 1961: 59; Halffter y Matthews 1966; Paulian 1988; Delgado y Deloya 1990; Gill 1991; Delgado 1995; Zunino y Halffter 1997; Zunino 2003; Krajcik 2006; Pulido-Herrera y Zunino 2007; Navarrete y Halffter 2008; Halffter y Halffter 2009; Barragán et al. 2011; Yanes-Gómez et al. 2015; Sánchez-de-Jesús et al. 2016; Zaragoza-Caballero y Pérez-Hernández 2017; Alvarado et al. 2018a; 2018b; Pinilla-Buitrago et al. 2018; Sánchez-Hernández et al. 2018; 2020; Capello y Halffter 2019; Sánchez-Huerta et al. 2019.

Patrón de distribución: Paleoamericano tropical.

Comentarios: Esta especie requiere de revisión taxonómica, ya que es posible que se trate de un complejo de especies crípticas. Se desconoce la ubicación actual de la mayor parte de la serie típica y del holotipo (28 individuos en total). Mediante una búsqueda en la colección entomológica personal del Dr. Gonzalo Halffter (2016) solamente fue posible encontrar un par de paratipos, pero no así al holotipo, por lo que es posible que este haya sido conservado en la colección personal del Padre F. S. Pereira. Hasta el momento, ha sido posible confirmar que un número reducido de paratipos se encuentra actualmente resguardado en la Colección Nacional de Insectos de la Universidad Nacional Autónoma de México, Ciudad de México; y en la Seção de Entomologia da Coleção Zoológica de la Universidad Federal de Mato Grosso, Cuiabá, Brasil. Es posible que $O$. carpophilus haya sido extirpado de la 
localidad tipo (Palomares, Oaxaca), ya que esta localidad se encuentra completamente deforestada en la actualidad, mientras que la especie en cuestión se distingue por ser un especialista que está fuertemente asociado a los frutos producidos por árboles de la selva tropical lluviosa (Pereira y Halffter 1961; Halffter y Halffter 2009; Sánchez-de-Jesús et al. 2016). Yanes-Gómez et al. (2015) reportan a O carpophilus de Veracruz. Sin embargo, este registro necesita corroborarse. Capello y Halffter (2019) citaron erróneamente a O. carpophilus de Jalisco.

21. O. cartwrighti Howden, 1973

BJS; Estados Unidos.

Onthophagus cartwrighti Howden, 1973: 329 (Figura 2); Anduaga y Halffter 1991; Krajcik 2006; Pulido-Herrera y Zunino 2007; Zunino y Halffter 2007; Moctezuma y Halffter 2019a; 2021.

Patrón de distribución: Paleoamericano del Altiplano Mexicano.

Comentarios: Esta especie ha sido reportada de Baja California Sur y California, por lo que es probable que se encuentre distribuida también en Baja California.

22. O. championi Bates, 1887

CHIS, OAX, VER; Guatemala, Honduras, El Salvador, Nicaragua, Costa Rica.

Onthophagus championi Bates, 1887: 74; Boucomont y Gillet 1927; Boucomont 1932; Blackwelder 1944; Gill 1991; Thomas 1993; Zunino y Halffter 1997; Kohlmann y Solís 2001; Hernández et al. 2003; Zunino 2003; Krajcik 2006; Horgan 2007; 2008; Kohlmann et al. 2007; Monaghan et al. 2007; Padilla-Hill y Halffter 2007; Pulido-Herrera y Zunino 2007; Solís y Kohlmann 2012; Gunter et al. 2016; Pinilla-Buitrago et al. 2018; Ramírez-Ponce et al. 2019; Ruíz-Pérez et al. 2019; Sánchez-Hernández et al. 2020; Moctezuma y Halffter 2021; Schwery y O'Meara 2021.

Patrón de distribución: Paleoamericano tropical.

23. O. chevrolati Harold, 1869

CDMX, EDOMEX, GTO, HGO, MICH, MOR, PUE, QRO, TLA, VER.

Onthophagus chevrolati Harold, 1869: 508; Boucomont y Gillet 1927; Boucomont 1932; Blackwelder 1944; Halffter 1962; Halffter y Matthews 1966; Edmonds y Halffter 1978; Zunino 1980; Zunino y Halffter 1988a; Martín-Piera y Lobo 1993; Martínez y Cruz 1999; Lobo y Halffter 2000; Navarrete-Heredia et al. 2001; Zunino 2003; Krajcik 2006; Escobar et al. 2007; Halffter et al. 2008; 2019; López-Guerrero y Zunino 2007; Pulido-Herrera y Zunino 2007; Jiménez-Sánchez et al. 2009; Aragón-García et al. 2011; Deloya-López 2011; Arriaga et al. 2012; Delgado et al. 2012b; Favila 2012; Morón et al. 2013; Arellano y Castillo-Guevara 2014; Arriaga-Jiménez et al. 2014; 2018; Naranjo-González y Víctor-Rosas 2015; Deloya et al. 2016; Pérez-Villamares et al. 2016; Márquez et al. 2017; Pinilla-Buitrago et al. 2018; Joaqui et al. 2019; Zaragoza-Caballero et al. 2019; Alvarado et al. 2020; Ortega-Martínez et al. 2020; 2021; Moctezuma y Halffter 2020a; $2020 \mathrm{~b}$.

Patrón de distribución: Paleoamericano de montaña.

Comentarios: Numerosos autores previos consideraban que esta especie contenía varias subespecies: chevrolati, retusus, omiltemius y longecarinatus. Estos taxa son considerados como especies válidas en la actualidad (ver Halffter et al. 2019; Moctezuma y Halffter 2020b).

24. O. chiapanecus Zunino y Halffter, 1988 CHIS.

Onthophagus chiapanecus Zunino y Halffter, 1988a: 126; Thomas 1993; Zunino 2003; Krajcik 2006; Pulido-Herrera y Zunino 2007; Zaragoza-Caballero y Pérez-Hernández 2017; Pinilla-Buitrago et al. 2018; Halffter et al. 2019; Joaqui et al. 2019; Moctezuma y Halffter 2020c; Sánchez-Hernández et al. 2020.

Patrón de distribución: Paleoamericano de montaña.

25. O. chilapensis Gasca-Álvarez, Zunino y Deloya, 2018

GRO.

Onthophagus chilapensis Gasca-Álvarez et al., 2018: 2122; Escobar-Hernández et al. 2019; Halffter et al. 2019; Moctezuma y Halffter 2020a.

Patrón de distribución: Paleoamericano de montaña. 26. O. chimalapensis Delgado y Mora-Aguilar, 2019 OAX.

Onthophagus chimalapensis Delgado y Mora-Aguilar, 2019: 586; Moctezuma y Halffter 2019b.

Patrón de distribución: Paleoamericano mesoamericano.

Comentarios: Esta especie podría encontrarse en los bosques de niebla del extremo occidental del estado de Chiapas.

27. O. chinantecus Moctezuma y Halffter, 2019 OAX.

Onthophagus chinantecus Moctezuma y Halffter, 2019b: 2; Moctezuma 2021.

Patrón de distribución: Paleoamericano tropical.

Comentarios: Esta especie probablemente se encuentra localizada en el extremo sur del estado de Veracruz, ya que fue descrita cerca de la frontera con Oaxaca, en la región del Uxpanapa. El macho de la especie se desconoce actualmente.

28. O. civitasorum Moctezuma y Halffter, 2020 DGO.

Onthophagus civitasorum Moctezuma y Halffter, 2020a: 2278 (Figura 3).

Patrón de distribución: Paleoamericano de montaña.

29. O. chryses Bates, 1887

OAX, VER; Guatemala, Costa Rica, Panamá.

Onthophagus chryses Bates, 1887: 76; Boucomont y Gillet 1927; Boucomont 1932; Blackwelder 1944; Howden y Young 1981; Deloya 1992b; 
Zunino y Halffter 1997; Kohlmann y Solís 2001; Zunino 2003; Krajcik 2006; Kohlmann et al. 2007; Pulido-Herrera y Zunino 2007; Escobar et al. 2008; Deloya-López 2011; Solís y Kohlmann 2012; Alvarado et al. 2014; Delgado y Curoe 2014; Arriaga-Jiménez et al. 2019.

Patrón de distribución: Paleoamericano mesoamericano.

Comentarios: Esta especie requiere de revisión taxonómica, ya que es posible que se trate de un complejo de especies crípticas.

30. O. clavijeroi Moctezuma, Rossini y Zunino, 2016 PUE.

Onthophagus clavijeroi Moctezuma et al., 2016a: 28; 2016b; Halffter et al. 2019; Joaqui et al. 2019; Moctezuma y Halffter 2020a.

Patrón de distribución: Paleoamericano de montaña.

Comentarios: Esta especie fue descrita originalmente como posiblemente necrófaga, pero no se ha descartado la posibilidad de que $O$. clavijeroi esté asociado a los cuerpos fructíferos de hongos macromicetos, tal como ocurre con otras especies relacionadas del complejo $O$. fuscus. Esta especie probablemente se encuentra en el estado de Tlaxcala, en la región de La Malinche. Sin embargo, la ausencia de muestreos con trampas cebadas con carroña y macromicetos en tal territorio no ha permitido confirmar esta expectativa.

31. O. coahuilae Zunino y Halffter, 1988

COA.

Onthophagus coahuilae Zunino y Halffter, 1988a: 94; Zunino 2003; Krajcik 2006; Pulido-Herrera y Zunino 2007; Halffter et al. 2019; Moctezuma y Halffter 2020b.

Patrón de distribución: Paleoamericano de montaña.

32. O. cochisus Brown, 1927

CHIH, DGO; Estados Unidos.

Onthophagus cochisus Brown, 1927: 132; Boucomont 1932; Leng y Mutchler 1933; Howden y Cartwright 1963; Zunino y Halffter 1988a; Morón y Deloya 1991; Navarrete-Heredia y Galindo-Miranda 1997; Anduaga 2000; Zunino 2003; Krajcik 2006; Pulido-Herrera y Zunino 2007; Halffter et al. 2019; Moctezuma y Halffter 2020 b.

Patrón de distribución: Paleoamericano de montaña.

33. O. coproides Horn, 1881

DGO; Estados Unidos.

Onthophagus coproides Horn, 1881: 79; Henshaw 1885; Schaeffer 1914; Leng 1920; Boucomont y Gillet 1927; Boucomont 1932; Howden y Cartwright 1963; Halffter y Matthews 1966; Edmonds y Halffter 1978; Zunino 1980; Zunino y Halffter 1988a 1988b 2007; Anduaga y Halffter 1991; Delgado 1999; Lobo y Halffter 1994; Zunino 2003; Dellacasa et al. 2004 (como O. cuboidalis); Krajcik 2006; Pulido-Herrera y Zunino 2007; Slay et al. 2012; Sánchez-Huerta et al. 2015; 2018; Arriaga-Jiménez et al. 2016;
Halffter 2017; 2019; Halffter y Morrone 2017; Bezanson y Floate 2019; Halffter et al. 2019; Morrone 2020.

Patrón de distribución: Paleoamericano de montaña. 34. O. corrosus Bates, 1887 CHIS, HGO, OAX, VER.

Onthophagus corrosus Bates, 1887: 78; Boucomont y Gillet 1927; Boucomont 1932; Blackwelder 1944; Pereira y Halffter 1961; Edmonds y Halffter 1978; Deloya 1992b; Martín-Piera y Lobo 1993; Thomas 1993; Halffter et al. 1995; 2007; 2008; Lobo y Halffter 2000; Arellano y Halffter 2003; Arellano et al. 2004; 2020; Krajcik 2006; Deloya et al. 2007; Escobar et al. 2007; Pulido-Herrera y Zunino 2007; Halffter y Halffter 2009; Deloya-López 2011; Delgado et al. 2012b; Barragán et al. 2014; Santiago-Molina et al. 2014; Huerta-Crespo y Cruz-Rosales 2016; Huerta et al. 2016; Márquez et al. 2017; Martínez et al. 2017; Pinilla-Buitrago et al. 2018; Miranda-Flores et al. 2020; Rivera et al. 2020; Sánchez-Hernández et al. 2020; Moctezuma 2021.

Patrón de distribución: Paleoamericano tropical.

Comentarios: Esta especie requiere de revisión taxonómica, ya que es posible que se trate de un complejo de especies crípticas.

35. O. coscineus Bates, 1887

CAM, CHIS; Belice, Panamá, Costa Rica, Colombia, Ecuador, Perú.

Onthophagus coscineus Bates, 1887: 79; Boucomont y Gillet 1927; Boucomont 1932; Blackwelder 1944; Howden y Young 1981; Hanski y Cambefort 1991; Delgado 1997; Escobar 2000; Kohlmann y Solís 2001; Medina et al. 2001; Celi et al. 2004; Krajcik 2006; Pulido-Herrera y Zunino 2007; Escobar et al. 2008; Navarrete y Halffter 2008; Arias-Buriticá et al. 2011; Barragán et al. 2011; Carvajal et al. 2011; Inward et al. 2011; Tarasov y Solodovnikov 2011; Pereira y Kohlmann 2012; Solís y Kohlmann 2012; Delgado y Curoe 2014; González-Alvarado y Medina 2015; González-Alvarado et al. 2015; Latha et al. 2016; Sánchez-de-Jesús et al. 2016; Chamorro et al. 2018; 2019; Capello y Halffter 2019; Sánchez-Hernández et al. 2020; Schwery y O'Meara 2021.

Patrón de distribución: Paleoamericano tropical.

Comentarios: Esta especie requiere de revisión taxonómica, ya que es posible que se trate de un complejo de especies crípticas. Pereira y Kohlmann (2012) utilizaron la grafía posterior incorrecta "coccineus" para referirse a esta especie.

36. O. crinitus Harold, 1869

CAM, CHIS, OAX, TAB, VER; Belice, Guatemala, El Salvador, Nicaragua, Costa Rica, Panamá, Colombia.

Onthophagus crinitus Harold, 1869: 510 (Figura 4); Boucomont y Gillet 1927; Boucomont 1932; Blackwelder 1944; Morón 1979; 1985; 
1987; 2004; Howden y Young 1981; Morón et al. 1985; Deloya 1992b; Halffter et al. 1992; Thomas 1993; Delgado 1997; Morón y Blackaller 1997; Zunino y Halffter 1997; Estrada et al. 1998; Palacios-Ríos et al. 1990; Escobar 2000; Barbero 2001; Kohlmann y Solís 2001; Medina et al. 2001; Estrada y Coates-Estrada 2002; Zunino 2003; Krajcik 2006; Kohlmann et al. 2007; Monaghan et al. 2007; Pulido-Herrera y Zunino 2007; Arellano et al. 2008; 2013; Navarrete y Halffter 2008; Barragán et al. 2011; Deloya-López 2011; Tarasov y Solodovnikov 2011; Solís y Kohlmann 2012; Alvarado et al. 2014; Delgado y Curoe 2014; Medina y González 2014; González-Alvarado y Medina 2015; González-Alvarado et al. 2015; Gunter et al. 2016; Sánchez-de-Jesús et al. 2016; Sánchez-Hernández et al. 2018; 2020; Capello y Halffter 2019; Ramírez-Ponce et al. 2019; Sánchez-Huerta et al. 2019; Rangel-Acosta et al. 2020; Moctezuma 2021.

Onthophagus panamensis Bates 1887: 68; Howden y Young 1981; Gill 1991; Krajcik 2006; Pulido-Herrera y Zunino 2007; Inward et al. 2011; Solís y Kohlmann 2012; Delgado y Curoe 2014; Rangel-Acosta y Martínez-Hernández 2017; Pinilla-Buitrago et al. 2018; Rivera et al. 2020; Schwery y O'Meara 2021.

Patrón de distribución: Paleoamericano tropical.

Comentarios: Inward et al. (2011) y Gunter et al. (2016) utilizaron la grafía posterior incorrecta "crinitis" para referirse a esta especie. Además, Inward et al. (2011) se refirieron a O. panamensis como variedad. Al parecer, esta especie se encuentra en proceso de revisión (Rossini et al. 2018a; 2018b)

37. O. cuboidalis Bates, 1887

OAX, PUE.

Onthophagus cuboidalis Bates, 1887: 79; Blackwelder 1944; Zunino y Halffter 1988a; 1988b 2007; Delgado 1999; Zunino 2003; Krajcik 2006; Pulido-Herrera y Zunino 2007; Sánchez-Huerta et al. 2015; 2018; Tarasov y Dimitrov 2016; Halffter 2017; 2019; Halffter y Morrone 2017; Halffter et al. 2019; Morrone 2020.

Patrón de distribución: Paleoamericano de montaña. 38. O. cuevensis Howden, 1973 SLP, TAM.

Onthophagus cuevensis Howden, 1973: 331; Zunino 1980; Zunino y Halffter 1988a; 1988b; 2007; Delgado 1999; Zunino 2003; Delgado y Montes de Oca 2005; García-Morales y Montes de Oca 2005; Krajcik 2006; Pulido-Herrera y Zunino 2007; Halffter et al. 2019.

Patrón de distribución: Paleoamericano de montaña. 39. O. cyanellus Bates, 1887

CHIS, HGO, OAX, PUE, TAM, VER; Guatemala, Honduras, El Salvador, Nicaragua, Costa Rica, Panamá. Onthophagus cyanellus Bates, 1887: 81 (Figuras
21-24); Boucomont 1932; Blackwelder 1944; Campos 1921; Edmonds y Halffter 1978; Howden y Young 1981; Zunino y Halffter 1988a; Deloya 1992b; Martín-Piera y Lobo 1993; Thomas 1993; Halffter et al. 1995; 2007; 2008; 2019; Maes 1998; Martínez y Cruz 1999; Lobo y Halffter 2000; Kohlmann y Solís 2001; Zunino 2003; Arellano et al. 2004; 2020; Delgado y Montes de Oca 2005; García-Morales y Montes de Oca 2005; Pineda et al. 2005; Krajcik 2006; Deloya et al. 2007; Escobar et al. 2007; Kohlmann et al. 2007; Pulido-Herrera y Zunino 2007; Muñoz-Hernández et al. 2008; Aragón-García et al. 2011; Creedy y Mann 2011; Deloya-López 2011; Martínez et al. 2011; Delgado et al. 2012a; 2012b; Rös et al. 2012; Solís y Kohlmann 2012; Morón et al. 2013; Barragán et al. 2014; Delgado y Curoe 2014; Huerta-Crespo y Cruz-Rosales 2016; Huerta et al. 2016; 2019; Márquez et al. 2017; Chamorro et al. 2018; 2019; Pini1la-Buitrago et al. 2018; Joaqui et al. 2019; Ramírez-Ponce et al. 2019; Ruíz-Pérez et al. 2019; Alvarado et al. 2020; Moctezuma y Halffter 2020b; 2020c; Sánchez-Hernández et al. 2020; Rivera et al. 2020; Joaqui et al. 2021; Moctezuma 2021.

Patrón de distribución: Paleoamericano mesoamericano.

Comentarios: Existen propuestas acerca de que O. cyanellus (Figuras 21-24) y O. mesoamericanus (Figuras 25-27) deberían ser considerados como sinónimos. Zunino y Halffter (1988a) y Pulido-Herrera y Zunino (2007) sugieren que son dos taxa distintos de nivel específico. Por el contrario, Kohlmann y Solís (2001) y Solís y Kohlmann (2012) proponen que O. mesoamericanus es el sinónimo más moderno de $O$. cyanellus. En este contexto, la idea de considerarlos como dos taxa distintos de nivel específico es apoyada por el presente estudio. La resurrección de $O$. mesoamericanus es tratada a detalle más adelante.

Chamorro et al. $(2018$; 2019) sugieren que $O$. cyanellus se encuentra en Ecuador. Sin embargo, la presencia de esta especie en Ecuador está en duda, ya que su registro data de hace 100 años (Campos 1921) y no ha sido reportada nuevamente en estudios posteriores. Es necesario revisar los especímenes reportados por Campos (1921) para corroborar la presencia de la especie en Sudamérica. Lamentablemente, se desconoce la ubicación actual de dichos especímenes, aunque es probable que algunos ejemplares se preserven en el National Museum of Natural History de los Estados Unidos (Chamorro comunicación personal).

40. O. cyclographus Bates, 1887

CAM, CHIS, OAX, QR, YUC; Guatemala.

Onthophagus cyclographus Bates, 1887: 79; 
Boucomont y Gillet 1927; Boucomont 1932; Blackwelder 1944; Pereira y Halffter 1961; Thomas 1993; Delgado 1997; Krajcik 2006; Pulido-Herrera y Zunino 2007; Navarrete y Halffter 2008; Barragán et al. 2011; Alvarado et al. 2018a; 2018b; Pinilla-Buitrago et al. 2018; Sánchez-Hernández et al. 2018; 2020; Capello y Halffter 2019.

Patrón de distribución: Paleoamericano tropical.

Comentarios: Esta especie requiere de revisión taxonómica, ya que es posible que se trate de un complejo de especies crípticas.

41. O. dubitabilis Howden y Génier, 2004 COL, JAL.

Onthophagus dubitabilis Howden y Génier, 2004: 60; Krajcik 2006; Pulido-Herrera y Zunino 2007; Moctezuma et al. 2016a; Arriaga-Jiménez et al. 2019.

Patrón de distribución: Paleoamericano del Altiplano Mexicano.

42. O. durangoensis Balthasar, 1939

CDMX, ¿COA?, COL, DGO, HGO, JAL, MICH, MOR, OAX, PUE, SIN, ¿SON?; Estados Unidos.

Onthophagus durangoensis Balthasar, 1939: 45; Martínez 1947; Krajcik 2006; Pulido-Herrera y Zunino 2007; Halffter et al. 2008; 2012; Rös et al. 2012; Morón et al. 2013; Huerta-Crespo y Cruz-Rosales 2016; Huerta et al. 2016; Trujillo-Miranda et al. 2016; Arriaga-Jiménez et al. 2019; Joaqui et al. 2021.

Onthophagus knulli Howden y Cartwright, 1963: 69; Delgado 1997; Zunino y Halffter 1997; Navarrete-Heredia et al. 2001; Edmonds 2004; Krajcik 2006; Pulido-Herrera y Zunino 2007; Halffter et al. 2008; Ortega-Martínez et al. 2016; Márquez et al. 2017; Hernández y Navarrete-Heredia 2018; sin. Arriaga-Jiménez et al. 2019: 12; Bezanson y Floate 2019; Rodríguez et al. 2019a; 2019b; Zaragoza-Caballero et al. 2019; Alvarado et al. 2020; Hernández et al. 2021.

Patrón de distribución: ¿Paleoamericano de montaña?

Comentarios: Esta especie requiere de revisión taxonómica, ya que es posible que se trate de un complejo de especies crípticas. Edmonds (2004), sugiere que una especie cercana a $O$. knulli se encuentra en Sonora, por lo que este registro requiere confirmarse.

43. O. etlaensis Kohlmann, Escobar-Hernández y Arriaga-Jiménez, 2019

OAX.

Onthophagus etlaensis Arriaga-Jiménez et al., 2019: 2; Salomão et al. 2021.

Patrón de distribución: Paleoamericano de montaña.

44. O. eulophus Bates, 1887

México (sin localidad específica).

Onthophagus eulophus Bates, 1887: 74; Boucomont y Gillet 1927; Boucomont 1932; Blackwelder 1944; Zunino 1981; 2003; Krajcik 2006; Puli-
do-Herrera y Zunino 2007; Moctezuma y Halffter 2021.

Patrón de distribución: Desconocido.

45. O.fuscus Boucomont, 1932

AGS, DGO, JAL, ZAC.

Onthophagus fuscus Boucomont, 1932: 316; Blackwelder 1944; Zunino y Halffter 1988a; Morón y Deloya 1991; Delgado 1997; Anduaga 2000; Lobo y Halffter 2000; Navarrete-Heredia et al. 2001; Zunino 2003; Morón 2004; Krajcik 2006; Pulido-Herrera y Zunino 2007; Moctezuma et al. 2016a; Halffter et al. 2019; Joaqui et al. 2019; Moctezuma y Halffter 2020a.

Patrón de distribución: Paleoamericano de montaña.

Comentarios: Autores previos consideraban que esta especie contenía varias subespecies: fuscus, canescens, mycetorum y parafuscus. Estos taxa son considerados como especies válidas en la actualidad (ver Joaqui et al. 2019).

46. O. gibsoni Howden y Génier, 2004 ¿HGO?, OAX, PUE.

Onthophagus gibsoni Howden y Génier, 2004: 66; Krajcik 2006; Pulido-Herrera y Zunino 2007; Halffter et al. 2008; Barragán et al. 2014; Ortega-Martínez et al. 2016; 2020; 2021; Márquez et al. 2017; Arriaga-Jiménez et al. 2019.

Patrón de distribución: Paleoamericano del Altiplano Mexicano.

Comentarios: Halffter et al. (2008), Barragán et al. (2014), Ortega-Martínez et al. (2016; 2020; 2021) y Márquez et al. (2017) citaron a esta especie del estado de Hidalgo. Sin embargo, este registro podría ser erróneo y requiere confirmación.

47. O. gilli Delgado y Howden, 2000 GRO.

Onthophagus gilli Delgado y Howden, 2000: 35; Krajcik 2006; Pulido-Herrera y Zunino 2007; Deloya y Covarrubias-Melgar 2014; Gasca-Álvarez et al. 2018; Escobar-Hernández et al. 2019; Halffter et al. 2019; Moctezuma y Halffter 2020a.

Patrón de distribución: Paleoamericano de montaña. 48. O. guatemalensis Bates, 1887

OAX, CHIS; Guatemala, Belice.

Onthophagus guatemalensis Bates, 1887: 73; Boucomont y Gillet 1927; Boucomont 1932; Blackwelder 1944; Howden y Cartwright 1963; Thomas 1993; Zunino 2003; Krajcik 2006; Pulido-Herrera y Zunino 2007; Sánchez-Hernández et al. 2020; Moctezuma 2021; Moctezuma y Halffter 2021.

Patrón de distribución: Paleoamericano mesoamericano.

49. O. halffteri Zunino, 1981 PUE, VER.

Onthophagus halffteri Zunino, 1981: 77 (Figura 5); Zunino y Halffter 1988a; 1988b; 2007; Delgado 1999; Zunino 2003; Krajcik 2006; Pulido-Herre- 
ra y Zunino 2007; Jiménez-Sánchez et al. 2009; Sánchez-Huerta et al. 2015; 2018; Halffter 2017; 2019; Halffter y Morrone 2017; Halffter et al. 2019; Morrone 2020.

Patrón de distribución: Paleoamericano de montaña.

Comentarios: Jiménez-Sánchez et al. (2009) citaron erróneamente a esta especie del Estado de México.

50. O. hidalgus Zunino y Halffter, 1988 HGO, NL, QRO, SLP.

Onthophagus hidalgus Zunino y Halffter, 1988a: 97; Zunino 2003; Krajcik 2006; Pulido-Herrera y Zunino 2007; Márquez et al. 2017; Halffter et al. 2019; Moctezuma y Halffter 2020b.

Patrón de distribución: Paleoamericano de montaña.

Comentarios: Esta especie requiere revisión taxonómica. Es posible que los registros de $O$. semiopacus de Hidalgo correspondan a esta especie o a $O$. potosinus.

51. O. hippopotamus Harold, 1869 CDMX, EDOMEX, MICH, PUE, VER.

Onthophagus hippopotamus Harold, 1869: 507; Boucomont y Gillet 1927; Boucomont 1932; Blackwelder 1944; Howden et al. 1956; Halffter y Matthews 1966; Zunino 1980; Paulian 1988; Zunino y Halffter 1988a; 1988b; 2007; Lobo y Halffter 1994; 2000; Delgado 1999; Martínez y Cruz 1999; Zunino 2003; Morón 2004; Krajcik 2006; López-Guerrero y Zunino 2007; PulidoHerrera y Zunino 2007; Jiménez-Sánchez et al. 2009; Deloya-López 2011; Favila 2012; Sánchez-Huerta et al. 2015; 2018; Deloya et al. 2016; Moctezuma et al. 2016a; Halffter 2017; 2019; Halffter y Morrone 2017; Moctezuma y Halffter 2020b; 2021; Halffter et al. 2019; Morrone 2020.

Patrón de distribución: Paleoamericano de montaña. 52. O. hoepfneri Harold, 1869

CHIS, EDOMEX, GRO, JAL, MICH, MOR, NAY, OAX, PUE, VER; Estados Unidos, El Salvador, Nicaragua, Costa Rica.

Onthophagus hoepfneri Harold, 1869: 512; Boucomont y Gillet 1927; Boucomont 1932; Robinson 1948; Howden y Cartwright 1963; Deloya 1992a; Halffter et al. 1992; 1995; 2012; Montes de Oca y Halffter 1995; Zunino y Halffter 1997; Deloya y Morón 1998; Maes 1998; Lobo y Halffter 2000; Halffter y Arellano 2002; Kohlmann y Solís 2001; Navarrete-Heredia et al. 2001; Zunino 2003; Pineda et al. 2005; Krajcik 2006; Deloya et al. 2007; 2013; 2016; Escobar et al. 2007; Horgan 2007; 2008; Kohlmann et al. 2007; Padilla-Hill y Halffter 2007; Pulido-Herrera y Zunino 2007; Andresen 2008a; 2008b; Mora-Aguilar y Montes de Oca 2009; Trevilla-Rebollar et al. 2010; Yanes-Gómez y Morón 2010; Deloya-López 2011; Favila 2012; Solís y Kohlmann 2012; Morón et al. 2013; Deloya y Covarrubias-Melgar 2014; Martínez et al. 2017;
Sánchez-Hernández et al. 2017; Arriaga-Jiménez et al. 2019; Bezanson y Floate 2019; Zaragoza-Caballero et al. 2019; Joaqui et al. 2021; Moctezuma 2021.

Onthophagus arizonensis Schaeffer, 1909: 382; 1914; Leng 1920; Boucomont y Gillet 1927; Boucomont 1932; Robinson 1948; sin. Howden y Cartwright 1963: 98; Zunino y Halffter 1997.

Patrón de distribución: Paleoamericano tropical.

Comentarios: Esta especie requiere de revisión taxonómica. Boucomont (1932), Gill (1991), Kohlmann et al. (2007), y Bezanson y Floate (2019) utilizaron la grafía posterior incorrecta "hopfne$r i$ ", mientras que Deloya (1992a) utilizó la grafía posterior incorrecta "hoëpfneri" para referirse a esta especie. Harold (1869), Boucomont y Gillet (1927), Howden y Cartwright (1963) y Martínez et al. (2017) utilizaron la grafía original incorrecta "höpfneri". De acuerdo con el artículo 32.5.2.1 del Código Internacional de Nomenclatura Zoológica (CINZ, 1999), los signos diacríticos o de otro tipo deben suprimirse de los epítetos específicos, excepto en nombres basados en una palabra alemana y publicados antes 1985, donde la diéresis debe borrarse de la vocal y tras ella debe insertarse la letra e, como es el caso de $O$. hoepfneri. Esta corrección probablemente fue realizada de forma original e implícita por Halffter et al. (1992), y señalada posteriormente por Pulido-Herrera y Zunino (2007).

53. O. howdeni Zunino y Halffter, 1988

OAX.

Onthophagus howdeni Zunino y Halffter, 1988a: 151; Krajcik 2006; Pulido-Herrera y Zunino 2007; Ramírez-Ponce et al. 2009; Arriaga-Jiménez et al. 2016; Escobar-Hernández et al. 2019; Halffter et al. 2019; Moctezuma y Halffter 2019a; Salomão et al. 2021.

Patrón de distribución: Paleoamericano de montaña.

Comentarios: Autores previos consideraban a esta especie como una subespecie de O. pseudoundulans (ver Halffter et al. 2019).

54. O. howdenorum Zunino y Halffter, 1988 OAX.

Onthophagus howdenorum Zunino y Halffter, 1988a: 142; Zunino 2003; Krajcik 2006; Pulido-Herrera y Zunino 2007; Escobar-Hernández et al. 2019; Halffter et al. 2019; Salomão et al. 2021.

Patrón de distribución: Paleoamericano de montaña. 55. O. igualensis Bates, 1887

CHIS, EDOMEX, GRO, HGO, JAL, MICH, MOR, PUE, ¿QR?, VER, ¿YUC?

Onthophagus igualensis Bates, 1887: 77; Boucomont y Gillet 1927; Boucomont 1932; Blackwelder 1944; Kohlmann y Sánchez 1984; Morón et al. 1986; 2013; Deloya 1992a; Zunino y Halffter 1997; Anduaga 2000; Halffter y Arellano 2002; Navarrete-Heredia et al. 2001; Zunino 2003; 
Krajcik 2006; Escobar et al. 2007; Padilla-Hill y Halffter 2007; Pulido-Herrera y Zunino 2007; Verdú et al. 2007; Andresen 2008a; 2008b; Arellano et al. 2008; 2013; Halffter et al. 2008; 2012; Trevilla-Rebollar et al. 2010; Barragán et al. 2011 2014; Rös et al. 2012; Deloya y Covarrubias-Melgar 2014; Deloya et al. 2016; Ortega-Martínez et al. 2016; Márquez et al. 2017; Sánchez-Hernández et al. 2017; 2020; Alvarado et al. 2018a; 2018b; Pinilla-Buitrago et al. 2018; Arriaga-Jiménez et al. 2019; Zaragoza-Caballero et al. 2019.

Patrón de distribución: Paleoamericano tropical.

Comentarios: Esta especie requiere de revisión taxonómica, ya que es posible que se trate de un complejo de especies crípticas.

56. O. incensus Say, 1835

CAM, CHIS, ¿EDOMEX?, GRO, HGO, JAL, MOR, OAX, QRO, SLP, TAM, VER; Estados Unidos, Guatemala, Honduras, El Salvador, Nicaragua, Costa Rica, Panamá.

Onthophagus incensus Say, 1835: 173 (Figura 6); Lacordaire 1856; Harold 1880; Blackwelder 1944; Howden y Cartwright 1963; Halffter y Matthews 1966; Vulcano y Pereira 1967; Howden y Young 1981; Deloya 1992b; Deloya et al. 1993; Martín-Piera y Lobo 1993; Thomas 1993; Halffter et al. 1995; 2007; 2008; Delgado 1997; Zunino y Halffter 1997; Martínez et al. 1998; 2011; Maes 1998; Martínez y Cruz 1999; Emlen y Nijhout 2000; Escobar 2000; Lobo y Halffter 2000; Kohlmann y Solís 2001; Martínez y Lumaret 2003; Zunino 2003; Arellano et al. 2004; 2017; 2014; 2020; Celi 2004; Delgado y Montes de Oca 2005; García-Morales y Montes de Oca 2005; Pineda et al. 2005; Krajcik 2006; Deloya et al. 2007; Escobar et al. 2007; Horgan 2007; Kohlmann et al. 2007; López-Guerrero y Zunino 2007; Pulido-Herrera y Zunino 2007; González-Maya y Mata-Lorenzen 2008; Navarrete y Halffter 2008; Halffter y Halffter 2009; Díaz et al. 2010; 2011; Huerta et al. 2010; 2016; 2019; Trevilla-Rebollar et al. 2010; Barragán et al. 2011; 2014; Carvajal et al. 2011; Deloya-López 2011; Tarasov y Solodovnikov 2011; Delgado et al. 2012b; Favila 2012; Morón y Márquez 2012; Rös et al. 2012; Solís y Kohlmann 2012; Huerta y García-Hernández 2013; Arriaga-Jiménez et al. 2014; Delgado y Curoe 2014; Deloya y Covarrubias-Melgar 2014; Morales-Morales et al. 2014; Santiago-Molina et al. 2014; Pérez-Cogollo et al. 2015; Young 2015; Huerta-Crespo y Cruz-Rosales 2016; Ortega-Martínez et al. 2016; Márquez et al. 2017; Chamorro et al. 2018; González-Tokman et al. 2018; Pinilla-Buitrago et al. 2018; Rossini et al. 2018a; Sánchez-Hernández et al. 2018; 2020; Bezanson y Floate 2019; Zaragoza-Caballero et al. 2019; Alvarado et al. 2020; Miranda-Flores et al. 2020; Rivera et al. 2020;
Salomão et al. 2020; Servín-Pastor et al. 2020; Joaqui et al. 2021; Schwery y O'Meara 2021.

Patrón de distribución: Paleoamericano tropical.

Comentarios: Es necesario actualizar la distribución de esta especie, ya que actualmente se encuentra en revisión (Rossini et al. 2018a; 2018b). Moctezuma et al. (2016a; 2016b) citaron erróneamente a esta especie de la región central de Puebla, ya que en una revisión reciente del espécimen reportado el autor del presente estudio comprobó que se trata de una hembra de $O$. mexicanus. Trevilla-Rebollar et al. (2010) citaron a esta especie del Estado de México, pero este registro necesita corroborarse ya que se encuentra fuera del área de distribución típica de la especie. Huerta y García-Hernández (2013) utilizaron la grafía posterior incorrecta "incestus" para referirse a esta especie.

57. O. inecolorum Moctezuma y Halffter, 2020 PUE.

Onthophagus inecolorum Moctezuma y Halffter, 2020a: 2282 (Figura 8).

Patrón de distribución: Paleoamericano tropical.

58. O. inflaticollis Bates, 1887

GRO.

Onthophagus inflaticollis Bates, 1887: 390; Boucomont y Gillet 1927; Boucomont 1932; Blackwelder 1944; Zunino 1980; Zunino y Halffter 1988a; Delgado y Howden 2000; Kohlmann y Solís 2001; Zunino 2003; Krajcik 2006; Pulido-Herrera y Zunino 2007; Deloya y Covarrubias-Melgar 2014; Gasca-Álvarez et al. 2018; Escobar-Hernández et al. 2019; Halffter et al. 2019; Moctezuma y Halffter 2020a.

Patrón de distribución: Paleoamericano de montaña. 59. O. iodiellus Bates, 1887 GRO.

Onthophagus iodiellus Bates, 1887: 390; Boucomont y Gillet 1927; Boucomont 1932; Blackwelder 1944; Delgado y Howden 2000; Zunino 2003; Krajcik 2006; Pulido-Herrera y Zunino 2007; Arriaga-Jiménez et al. 2019.

Patrón de distribución: Paleoamericano tropical.

Comentarios: Pulido-Herrera y Zunino (2007) erróneamente indican que esta especie se encuentra distribuida en Durango, México.

60. O. istmenus Moctezuma, Sánchez-Huerta y Halffter, 2020 OAX.

Onthophagus istmenus Moctezuma et al., 2020: 281 (Figura 7); Moctezuma 2021.

Patrón de distribución: Paleoamericano tropical.

Comentarios: Esta especie probablemente se encuentra localizada en el extremo sur del estado de Veracruz, ya que fue descrita cerca de la frontera con Oaxaca, en la región del Uxpanapa. Moctezuma et al. (2020) incorrectamente se refirieron a esta especie dentro del patrón de distribución neotropical típico. 
61. O. ixtepecorum Moctezuma y Halffter, 2020 OAX.

Onthophagus ixtepecorum Moctezuma y Halffter, 2020b: 251.

Patrón de distribución: Paleoamericano tropical.

Comentarios: Autores previos consideraban a esta especie como una población de $O$. chevrolati (ver Moctezuma y Halffter 2020b).

62. O. ixtlanensis Moctezuma y Halffter, 2020 OAX.

Onthophagus ixtlanensis Moctezuma y Halffter, 2020b: 252.

Patrón de distribución: Paleoamericano de montaña.

Comentarios: Autores previos consideraban a esta especie como una población de $O$. chevrolati (ver Moctezuma y Halffter 2020b).

63. O. jaliscensis Zunino y Halffter, 1988 JAL.

Onthophagus jaliscensis Zunino y Halffter, 1988a: 153; Navarrete-Heredia et al. 2001; Krajcik 2006; Pulido-Herrera y Zunino 2007; Arriaga-Jiménez et al. 2016; Halffter et al. 2019.

Patrón de distribución: Paleoamericano tropical.

Comentarios: Esta especie era considerada como una subespecie de $O$. tarascus por autores previos (ver Halffter et al. 2019).

64. O. landolti Harold, 1880

CAM, CHIS, HGO, JAL, QR, VER, YUC; Estados Unidos, Guatemala, Belice, El Salvador, Nicaragua, Costa Rica, Panamá, Colombia, Venezuela.

Onthophagus landolti landolti Harold, 1880: 34; Boucomont y Gillet 1927; Boucomont 1932; Blackwelder 1944; Edmonds y Halffter 1978; Howden y Gill 1987; Gill 1991; Deloya 1992b; Halffter et al. 1992; 2008; 2012; Thomas 1993; Halffter et al. 1995; Escobar 1997; 2000; Zunino y Halffter 1997; Estrada et al. 1998; Maes 1998; Halffter y Arellano 2002; Kohlmann y Solís 2001; Medina et al. 2001; Navarrete-Heredia et al. 2001; Estrada y Coates-Estrada 2002; Bustos y Lopera 2003; Hernandéz et al. 2003; Zunino 2003; Krajcik 2006; Andresen 2008a; 2008b; Horgan 2007; 2008; Kohlmann et al. 2007; Noriega et al. 2007; Padilla-Hill y Halffter 2007; Pulido-Herrera y Zunino 2007; Reyes-Novelo et al. 2007; Quiroz-Rocha et al. 2008; Díaz et al. 2010; 2011; Granados et al. 2010; Martínez-H et al. 2010; Barragán et al. 2011; 2014; Deloya-López 2011; Cultid-Medina et al. 2012; Delgado et al. 2012b; Favila 2012; 2017; Solís et al. 2011; Cultid-Medina et al. 2012; Solís y Kohlmann 2012; Arellano et al. 2013; 2017; Basto-Estrada et al. 2013; Alvarado et al. 2014; 2018a; 2018b; 2020; Delgado y Curoe 2014; Medina y González 2014; Santiago-Molina et al. 2014; González-Alvarado y Medina 2015; González-Alvarado et al. 2015; Hernández-Córdoba et al. 2015; Pérez-Cogollo et al. 2015; 2018; Montoya-Molina et al. 2015;
González-Tokman et al. 2017; 2018; Márquez et al. 2017; Rangel-Acosta y Martínez-Hernández 2017; Sánchez-Hernández et al. 2017; 2018; 2020; Amell-Caez et al. 2019; Capello y Halffter 2019; Rodríguez-López et al. 2019; Rodríguez-Vivas et al. 2019; Miranda-Flores et al. 2020; Pablo-Cea et al. 2020; Rangel-Acosta et al. 2020; Salomão et al. 2020; Joaqui et al. 2021. Onthophagus landolti texanus Schaeffer, 1914: 299; Howden y Cartwright 1963; Halffter y Matthews 1966; Montes de Oca y Halffter 1995; Zunino y Halffter 1997; Delgado y Montes de Oca 2005; García-Morales y Montes de Oca 2005; Krajcik 2006; Pulido-Herrera y Zunino 2007; Halffter et al. 2008; Pérez-Cogollo et al. 2015; Arellano et al. 2017; Martínez et al. 2017; Rangel-Acosta et al. 2018; Bezanson y Floate 2019.

Patrón de distribución: Paleoamericano tropical.

Comentarios: Es necesario revisar taxonómicamente a esta especie, ya que podría tratarse de un complejo de especies crípticas sin resolver que incluye al menos dos especies distintas. Cuando la subespecie $O$. l. texanus fue descrita, se omitió la revisión de sus estructuras genitales y su comparación con $O$. l. landolti. Estas estructuras tienen una gran importancia para la determinación y delimitación de especies en la taxonomía actual de los Onthophagus de América. Se recomienda que estudios futuros indiquen a cuál de las dos subespecies hacen referencia.

65. O. lecontei Harold, 1871

AGS, CDMX, DGO, EDOMEX, GTO, HGO, JAL, MICH, MOR, NL, OAX, PUE, QRO, SLP, TLA, VER, ZAC; Estados Unidos.

Onthophagus lecontei Harold, 1871: 115 (Figura 9); Boucomont y Gillet 1927; Boucomont 1932; Blackwelder 1944; Howden y Cartwright 1963; Deloya et al. 1993; 2016; Delgado 1997; Zunino y Halffter 1997; Martínez et al. 1999; Anduaga 2000; Lobo y Halffter 2000; Morón et al. 2000; 2013; Zunino 2003; Howden y Génier 2004; Krajcik 2006; Pulido-Herrera y Zunino 2007; Verdú et al. 2007; Halffter et al. 2008; 2012; Arellano et al. 2009; 2017; Jiménez-Sánchez et al. 2009; Yanes-Gómez y Morón 2010; Barragán et al. 2011; Arriaga et al. 2012; Delgado et al. 2012b; Arellano y Castillo-Guevara 2014; Zamora-Vuelvas et al. 2014; González-Hernández et al. 2015; Lizardo y Castellano-Vargas 2016; Moctezuma et al. 2016a; 2016b; Trujillo-Miranda et al. 2016; Arriaga-Jiménez et al. 2018; 2019; Márquez et al. 2017; Hernández y Navarrete-Heredia 2018; Pinilla-Buitrago et al. 2018; Rodríguez et al. 2019a; 2019b; Zaragoza-Caballero et al. 2019; Ortega-Martínez et al. 2020; 2021; Hernández et al. 2021.

Patrón de distribución: Paleoamericano del Altiplano Mexicano.

Comentarios: Esta especie requiere de revisión ta- 
xonómica. Después de revisar los ejemplares colectados por Lizardo y Castellano-Vargas (2016), el autor del presente estudio corroboró que Onthophagus sp 2 y Onthophagus sp 3 corresponden a $O$. lecontei.

66. O. longecarinatus Zunino y Halffter, 1988 NL, QRO.

Onthophagus longecarinatus Zunino y Halffter, 1988a: 112; Krajcik 2006; Pulido-Herrera y Zunino 2007; Halffter et al. 2019; Moctezuma y Halffter 2020b.

Patrón de distribución: Paleoamericano tropical.

Comentarios: Autores previos consideraban a esta especie como una subespecie de $O$. chevrolati (ver Halffter et al. 2019).

67. O. longimanus Bates, 1887

CAM, CHIS, HGO, TAM, VER, YUC; Belice, Guatemala, Honduras.

Onthophagus longimanus Bates, 1887: 76; Boucomont y Gillet 1927; Boucomont 1932; Blackwelder 1944; Deloya 1992b; Zunino y Halffter 1997; Zunino 2003; Delgado y Montes de Oca 2005; García-Morales y Montes de Oca 2005; Krajcik 2006; Pulido-Herrera y Zunino 2007; Halffter et al. 2008; Creedy y Mann 2011; Deloya-López 2011; Inward et al. 2011; Breeschoten et al. 2016; Márquez et al. 2017; Sánchez-Hernández et al. 2017; 2018; 2020; Alvarado et al. 2018a; 2018b; Arriaga-Jiménez et al. 2019; Capello y Halffter 2019.

Patrón de distribución: Paleoamericano tropical.

68. O. luismargaritorum Delgado, 1995

CHIS, VER.

Onthophagus luismargaritorum Delgado, 1995: 57; Delgado y Pensado 1998; Delgado et al. 2006; Krajcik 2006; Pulido-Herrera y Zunino 2007; Zaragoza-Caballero y Pérez-Hernández 2017; Moctezuma et al. 2020.

Patrón de distribución: Paleoamericano tropical.

69. O. marginicollis Harold, 1880

CHIS, GRO, OAX, VER; Guatemala, Cuba, El Salvador, Nicaragua, Costa Rica, Panamá, Colombia, Venezuela, Guyana, Ecuador, Perú, Brasil, Bolivia.

Onthophagus marginicollis Harold, 1880: 31; Boucomont y Gillet 1927; Boucomont 1932; Blackwelder 1944; Pereira y Halffter 1961; Halffter y Matthews 1966; Vulcano y Pereira 1967; Howden y Young 1981; Blanco-Casanova 1988; Gill 1991; Thomas 1993; Delgado 1997; Escobar 1997; 2000; Zunino y Halffter 1997; Maes 1998; Emlen y Nijhout 2000; Vaz-de-Mello 2000; Barbero 2001; Kohlmann y Solís 2001; Medina et al. 2001; Bustos y Lopera 2003; Hernandéz et al. 2003; Zunino 2003; Krajcik 2006; Horgan 2007; 2008; Kohlmann et al. 2007; Noriega et al. 2007; 2016; Padilla-Hill y Halffter 2007; Pulido-Herrera y Zunino 2007; Escobar et al. 2008; Larsen et al. 2008; Orozco y Pérez 2008; Martínez et al. 2009; Medina y Pulido 2009;
Granados et al. 2010; Martínez-H et al. 2010; Villamarín-Cortez 2010; Arias-Buriticá et al. 2011; Deloya-López 2011; Inward et al. 2011; Solís et al. 2011; Tarasov y Solodovnikov 2011; Cultid-Medina et al. 2012; Pereira y Kohlmann 2012; Solís y Kohlmann 2012; Delgado y $\mathrm{Cu}-$ roe 2014; Deloya y Covarrubias-Melgar 2014; Morales-Morales et al. 2014; González-Alvarado y Medina 2015; González-Alvarado et al. 2015; Montoya-Molina et al. 2015; Ratcliffe et al. 2015; Montoya-Molina et al. 2016; Ramírez-Restrepo y Halffter 2016; Rangel-Acosta et al. 2016; 2018; 2020; Tovar et al. 2016; Rangel-Acosta y Martínez-Hernández 2017; Chamorro et al. 2018; 2019; Giraldo-Echeverri et al. 2018; Martínez-Revelo et al. 2018; Rossini et al. 2018a; Amell-Caez et al. 2019; Montes-Rodríguez et al. 2019; Ortega-Echeverría et al. 2019; Sánchez-Hernández et al. 2020; Noriega et al. 2021; Schwery y O'Meara 2021; Whitworth et al. 2021.

Patrón de distribución: Paleoamericano tropical.

Comentarios: Granados et al. (2010) utilizaron la grafía posterior incorrecta "marginicoulis", mientras Whitworth et al. (2021) utilizaron la grafía posterior incorrecta "marginincollis" para referirse a esta especie. La distribución de esta especie necesita actualizarse, ya que se encuentra actualmente bajo revisión (Rossini et al. 2018a; 2018b).

70. O. mariozuninoi Delgado, Navarrete y Blackaller, 1993

EDOMEX, JAL, MICH, MOR.

Onthophagus mariozuninoi Delgado et al., 1993: 122; Delgado 1997; Navarrete-Heredia y Galindo-Miranda 1997; Zunino y Halffter 1997; Anduaga 2000; Navarrete-Heredia et al. 2001; Zunino 2003; Krajcik 2006; Pulido-Herrera y Zunino 2007; Trevilla-Rebollar et al. 2010; Zamora-Vuelvas et al. 2014; Deloya et al. 2016; Moctezuma et al. 2016a; Zaragoza-Caballero y Pérez-Hernández 2017; Arriaga-Jiménez et al. 2019; Zaragoza-Caballero et al. 2019.

Patrón de distribución: Paleoamericano de montaña.

71. O. martinpierai Moctezuma, Rossini y Zunino, 2016

HGO, PUE.

Onthophagus martinpierai Moctezuma et al., 2016a: 38; 2016b; Arriaga-Jiménez et al. 2019.

Comentarios: Moctezuma et al. (2016) indicaron que algunos paratipos de esta especie fueron depositados en la Canadian National Collection of Insects, Arachnids and Nematodes, Ontario, $\mathrm{Ca}-$ nadá. Sin embargo, esta afirmación es errónea, ya que los paratipos fueron depositados en el Canadian Museum of Nature, Ontario, Canadá (2 hembras). Después de la publicación original de la especie; algunos paratipos fueron depositados adicionalmente en las siguientes colecciones: 
Seção de Entomologia da Coleção Zoológica de la Universidad Federal de Mato Grosso, Cuiabá, Brasil (2 hembras); Colección Personal de Leonardo Delgado (1 macho, 1 hembra); Colección Personal de José Luis Sánchez-Huerta (1 hembra); y Colección Entomológica IEXA "Dr. Miguel Angel Morón Ríos" del Instituto de Ecología, A.C. (2 hembras).

Patrón de distribución: Paleoamericano del Altiplano Mexicano.

72. O. mateui Moctezuma y Halffter, 2020

OAX.

Onthophagus mateui Moctezuma y Halffter, 2020b: 253.

Patrón de distribución: Paleoamericano de montaña.

Comentarios: Autores previos consideraban a esta especie como una población de $O$. chevrolati (ver Moctezuma y Halffter 2020b).

73. O. maya Zunino, 1981

CAM, CHIS, OAX, QR, VER; Belice, Guatemala.

Onthophagus maya Zunino, 1981: 78 (Figura 10); Morón 1985; 1987; 1993; Morón et al. 1985; Delgado y Deloya 1990; Thomas 1993; Delgado 1995; Zunino y Halffter 1997; Delgado y Pensado 1998; Palacios-Ríos et al. 1990; Zunino 2003; Delgado et al. 2006; Krajcik 2006; Pulido-Herrera y Zunino 2007; Navarrete y Halffter 2008; Halffter y Halffter 2009; Barragán et al. 2011; Deloya-López 2011; Alvarado et al. 2014; Sánchez-de-Jesús et al. 2016; Zaragoza-Caballero y Pérez-Hernández 2017; Pinilla-Buitrago et al. 2018; Sánchez-Hernández et al. 2018; 2020; Capello y Halffter 2019; Sánchez-Huerta et al. 2019; Rivera et al. 2020; Joaqui et al. 2021; Moctezuma 2021.

Patrón de distribución: Paleoamericano de tropical.

74. O. mcclevei Howden y Génier, 2004

SON.

Onthophagus mcclevei Howden y Génier 2004: 54; Krajcik 2006; Pulido-Herrera y Zunino 2007; Moctezuma y Halffter 2021.

Patrón de distribución: Paleoamericano del Altiplano Mexicano.

75. O. mexicanus Bates, 1887

CDMX, COL, DGO, EDOMEX, GRO, GTO, HGO,

JAL, MICH, MOR, NAY, OAX, PUE, QRO, SIN, SLP, TLA, VER, ZAC.

Onthophagus mexicanus Bates, 1887: 72 (Figura 11); Boucomont y Gillet 1927; Boucomont 1932; Blackwelder 1944; Halffter y Matthews 1966; Kohlmann y Sánchez 1984; Deloya et al. 1993; 2016; Thomas 1993; Zunino y Halffter 1997; Vaz-de-Mello et al. 1998; Morón et al. 2000; 2013; Navarrete-Heredia et al. 2001; Estrada y Coates-Estrada 2002; Zunino 2003; Krajcik 2006; Pulido-Herrera y Zunino 2007; Verdú et al. 2007; Halffter et al. 2008; 2012; Jiménez-Sánchez et al. 2009; Trevilla-Rebollar et al. 2010; Yanes-Gómez y Morón 2010; Aragón-García et al. 2011; Barragán et al. 2011; 2014; Deloya-López 2011; Arriaga et al. 2012; Delgado et al. 2012b; Favila 2012; Arellano y Castillo-Guevara 2014; Deloya y Covarrubias-Melgar 2014; Zamora-Vuelvas et al. 2014; Naranjo-González y Víctor-Rosas 2015; Moctezuma et al. 2016a; 2016b; Ortega-Martínez et al. 2016; 2020; 2021; Trujillo-Miranda et al. 2016; Carrillo-Ruíz et al. 2017; Márquez et al. 2017; Arriaga-Jiménez et al. 2018; Hernández y Navarrete-Heredia 2018; Kohlmann et al. 2018; Pinilla-Buitrago et al. 2018; Escobar-Hernández et al. 2019; Ramírez-Ponce et al. 2019; Zaragoza-Caballero et al. 2019; Hernández et al. 2021; Salomão et al. 2021.

Patrón de distribución: Paleoamericano del Altiplano Mexicano.

Comentarios: Naranjo-González y Víctor-Rosas (2015) erróneamente reportaron como O. mexicanus a una hembra que probablemente corresponde a $O$. chevrolati. Thomas (1993) y Deloya et al. (2016) citaron erróneamente a esta especie para el estado de Chiapas.

76. O. mextexus Howden y Cartwright, 1970 GTO, HGO, NL, PUE, SLP, VER; Estados Unidos.

Onthophagus mextexus Howden y Cartwright, 1970: 54; Halffter et al. 1995; 2007; Deloya et al. 2007; Escobar et al. 2007; Pulido-Herrera y Zunino 2007; Márquez et al. 2017; Arriaga-Jiménez et al. 2019.

Onthophagus monticolus Howden y Cartwright, 1963: 61 (non Onthophagus monticolus Paulian, 1937); Howden y Cartwright 1970; Pulido-Herrera y Zunino 2007; Aragón-García et al. 2011; Deloya-López 2011.

Patrón de distribución: Paleoamericano de montaña.

Comentarios: Esta especie requiere de revisión taxonómica, ya que es posible que se trate de un complejo de especies crípticas.

77. O. moroni Zunino y Halffter, 1988

PUE.

Onthophagus moroni Zunino y Halffter, 1988b: 19 (Figura 12); 2007; Delgado 1999; Zunino 2003; Krajcik 2006; Halffter y Deloya 1997; López-Guerrero y Zunino 2007; Pulido-Herrera y Zunino 2007; Slay et al. 2012; Morón et al. 2013; Halffter et al. 2019; Rivera-Gasperín et al. 2021.

Patrón de distribución: Paleoamericano de montaña.

78. O. mycetorum Zunino y Halffter, 1988 EDOMEX, MOR.

Onthophagus mycetorum Zunino y Halffter, 1988a: 80; Delgado 1997; Navarrete-Heredia y Galindo-Miranda 1997; Anduaga 2000; Krajcik 2006; Pulido-Herrera y Zunino 2007; Jiménez-Sánchez et al. 2009; Moctezuma et al. 2016a; Halffter et al. 2019; Joaqui et al. 2019; Moctezuma y 
Halffter 2020a.

Patrón de distribución: Paleoamericano de montaña.

Comentarios: Autores previos consideraban a esta especie como una subespecie de $O$. fuscus (ver Joaqui et al. 2019).

79. O. nasicornis Guérin-Méneville, 1855 ¿CHIS?, HGO, PUE, VER.

Onthophagus nasicornis Guérin-Méneville, 1855: 511; Boucomont y Gillet 1927; Boucomont 1932; Blackwelder 1944; Morón 1979; 1985; 1986; 2004; Morón et al. 1985; 2013; Delgado y Deloya 1990; Deloya 1992b; Estrada et al. 1993; 1998; Thomas 1993; Delgado 1995; Halffter et al. 1995; 2007; 2008; Zunino y Halffter 1997; Estrada y Coates-Estrada 2002; Carrillo-Ruíz y Morón 2003; Zunino 2003; Pineda et al. 2005; Krajcik 2006; Deloya et al. 2007; Escobar et al. 2007; Halffter 2007; Padilla-Hill y Pulido-Herrera y Zunino 2007; Muñoz-Hernández et al. 2008; Halffter y Halffter 2009; Aragón-García et al. 2011; Deloya-López 2011; Delgado et al. 2012b; Deloya-López y López-Huicochea 2012; Rös et al. 2012; Alvarado et al. 2014; Morón y Arce-Pérez 2016; Márquez et al. 2017; Pinilla-Buitrago et al. 2018; Arellano et al. 2020; Sánchez-Hernández et al. 2020.

Patrón de distribución: Paleoamericano tropical.

Comentarios: Esta especie requiere de revisión taxonómica, ya que es posible que se trate de un complejo de especies crípticas. Sánchez-Hernández et al. (2020) sugieren que la presencia de esta especie es dudosa en el estado de Chiapas.

80. O. navarretorum Delgado y Capistan, 1996 MOR.

Onthophagus navarretorum Delgado y Capistan, 1996: 157; Anduaga 2000; Zunino 2003; Krajcik 2006; Zaragoza-Caballero y Pérez-Hernández 2017; Halffter et al. 2019; Joaqui et al. 2019; Zaragoza-Caballero et al. 2019; Moctezuma y Halffter 2020a.

Patrón de distribución: Paleoamericano de montaña.

81. O. neofuscus Moctezuma y Halffter, 2020

JAL.

Onthophagus neofuscus Moctezuma y Halffter, 2020a: 2281.

Patrón de distribución: Paleoamericano de montaña.

Comentarios: Autores previos consideraban a esta especie como una población de $O$. pseudofuscus (ver Moctezuma y Halffter 2020a).

82. O. neomirabilis Howden, 1973

OAX.

Onthophagus neomirabilis Howden, 1973: 334; Zunino 1981; Delgado y Deloya 1990; Howden y Gill 1993; Delgado 1997; Zunino y Halffter 1997; Génier y Howden 1999; Zunino 2003; Krajcik 2006; Pulido-Herrera y Zunino 2007; Génier 2017; Delgado y Mora-Aguilar 2019.

Patrón de distribución: Paleoamericano mesoamericano.
83. O. nitidior Bates, 1887

COL, DGO, EDOMEX, GRO, JAL, MICH, MOR, NAY, OAX, SIN.

Onthophagus nitidior Bates, 1887: 67; Boucomont y Gillet 1927; Boucomont 1932; Blackwelder 1944; Kohlmann y Sánchez 1984; Deloya et al. 1993; 2016; Thomas 1993; Delgado 1997; Zunino y Halffter 1997; Palacios-Ríos et al. 1990; Navarrete-Heredia et al. 2001; Zunino 2003; Krajcik 2006; Pulido-Herrera y Zunino 2007; Quiroz-Rocha et al. 2008; Jiménez-Sánchez et al. 2009; Deloya y Covarrubias-Melgar 2014; Breeschoten et al. 2016; Hernández y Navarrete-Heredia 2018; Pinilla-Buitrago et al. 2018; Rossini et al. 2018a; Rodríguez et al. 2019a; 2019b; Zaragoza-Caballero et al. 2019; Sánchez-Hernández et al. 2020.

Patrón de distribución: Paleoamericano tropical.

Comentarios: Es necesario actualizar la distribución de esta especie, ya que actualmente se encuentra en revisión (Rossini et al. 2018a, 2018b). De acuerdo con Sánchez-Hernández et al. (2020), el registro de $O$. nitidior para Chiapas reportado por Palacios-Ríos et al. (1990) y de forma subsecuente por Thomas (1993) es erróneo. Es posible que este registro corresponda a $O$. incensus u $O$. batesi.

84. O. nudifrons Balthasar, 1939

Sur de México (sin localidad específica).

Onthophagus nudifrons Balthasar, 1939: 46; Pulido-Herrera y Zunino 2007.

Patrón de distribución: Desconocido.

Comentarios: Pulido-Herrera y Zunino (2007) sugieren que esta especie debe ser considerada como nomen inquirendum, ya que su descripción y datos de colecta parecían ser "muy vagos" para tales autores. Contrario a la opinión de Pulido-Herrera y Zunino (2007), la descripción original de Balthasar (1939) es bastante detallada y sugiere que esta especie podría estar relacionada con O. orpheus Panzer, 1794 y O. mexicanus. El caso de $O$. nudifrons será tratado en un estudio subsecuente. El holotipo de esta especie (Figura 28) se encuentra depositado actualmente en el Národní Muzeum de Praga, Republica Checa.

85. O. oaxacanus Zunino y Halffter, 1988

OAX.

Onthophagus oaxacanus Zunino y Halffter, 1988a: 139; Krajcik 2006; Pulido-Herrera y Zunino 2007; Escobar-Hernández et al. 2019; Halffter et al. 2019; Salomão et al. 2021.

Patrón de distribución: Paleoamericano de montaña.

Comentarios: Autores previos consideraban a esta especie como una subespecie de $O$. undulans (ver Halffter et al. 2019).

86. O. omiltemius Bates, 1887 GRO.

Onthophagus omiltemius Bates, 1887a: 111; Pulido-Herrera y Zunino 2007; Deloya y Covarru- 
bias-Melgar 2014; Gasca-Álvarez et al. 2018; Halffter et al. 2019; Moctezuma y Halffter 2020b.

Patrón de distribución: Paleoamericano de montaña.

Comentarios: Autores previos consideraban a esta especie como una subespecie de $O$. chevrolati (ver Halffter et al. 2019).

87. O. orizabensis Moctezuma, Joaqui y Sánchez-Huerta, 2019

PUE, VER.

Onthophagus orizabensis Joaqui et al., 2019: 153; 2021; Halffter et al. 2019; Moctezuma y Halffter 2020a.

Patrón de distribución: Paleoamericano de montaña.

Comentarios: Autores previos confundieron a esta especie con $O$. chevrolati y $O$. retusus (ver Joaqui et al. 2019).

88. O. padrinoi Delgado, 1999

JAL.

Onthophagus padrinoi Delgado, 1999: 34; Navarrete-Heredia et al. 2001; Krajcik 2006; Pulido-Herrera y Zunino 2007; Zunino y Halffter 2007; Sánchez-Huerta et al. 2015; 2018; Halffter 2017; 2019; Halffter y Morrone 2017; Halffter et al. 2019; Morrone 2020.

Patrón de distribución: Paleoamericano de montaña.

89. O. parafuscus Zunino y Halffter, 2005

HGO, PUE, VER.

Onthophagus parafuscus Zunino y Halffter, 2005: 149; Escobar et al. 2007; Pulido-Herrera y Zunino 2007; Halffter et al. 2008; 2019; Deloya-López 2011; Favila 2012; Moctezuma et al. 2016a; Márquez et al. 2017; Joaqui et al. 2019; Moctezuma y Halffter 2020a.

Onthophagus orientalis Zunino y Halffter, 1988a: 82 (non Onthophagus orientalis Harold, 1868); Zunino y Halffter 2005; Krajcik 2006; Pulido-Herrera y Zunino 2007; Márquez et al. 2017; Joaqui et al. 2019.

Patrón de distribución: Paleoamericano de montaña.

Comentarios: Autores previos citaron a esta especie como $O$. fuscus, $O$. fuscus orientalis y $O$. fuscus parafuscus (ver Joaqui et al. 2019).

90. O. pedester Howden y Génier, 2004 OAX.

Onthophagus pedester Howden y Génier, 2004: 58; Gasca-Álvarez et al. 2018; Escobar-Hernández et al. 2019; Arriaga-Jiménez et al. 2019.

Patrón de distribución: Paleoamericano de montaña. 91. O. petenensis Howden y Gill, 1993 ¿OAX?, CHIS; Belice, Guatemala, Honduras.

Onthophagus petenensis Howden y Gill, 1993: 1096; Zunino y Halffter 1997; Krajcik 2006; Pulido-Herrera y Zunino 2007; Creedy y Mann 2011; Alvarado et al. 2014; Génier 2017; Moctezuma y Halffter $2019 b$.

Patrón de distribución: Paleoamericano tropical.

Comentarios: Esta especie requiere de revisión taxonómica. Alvarado et al. (2014) reportaron a esta especie de Oaxaca. Sin embargo, este registro necesita confirmación ya que los individuos reportados por tal autor podrían corresponder a $O$. chinantecus. Para el presente estudio se intentó verificar la determinación taxonómica de tal registro; sin embargo, la ubicación actual de los especímenes reportados por Alvarado et al. (2014) es desconocida.

92. O. potosinus Moctezuma y Halffter, 2020 SLP.

Onthophagus potosinus Moctezuma y Halffter, 2020b: 255.

Patrón de distribución: Paleoamericano de montaña.

Comentarios: Autores previos consideraban a esta especie como una población de $O$. chevrolati (ver Moctezuma y Halffter 2020b).

93. O. pseudofuscus Zunino y Halffter, 1988 DGO, SIN.

Onthophagus pseudofuscus Zunino y Halffter, 1988a: 85 (Figura 13); Navarrete-Heredia y Galindo-Miranda 1997; Anduaga 2000; Navarrete-Heredia et al. 2001; Zunino 2003; Krajcik 2006; Pulido-Herrera y Zunino 2007; Moctezuma et al. 2016a; Joaqui et al. 2019; Moctezuma y Halffter 2020a.

Patrón de distribución: Paleoamericano de montaña.

94. O. pseudoguatemalensis Moctezuma y Halffter, 2021

JAL.

Onthophagus pseudoguatemalensis Moctezuma y Halffter, 2021.

Patrón de distribución: Paleoamericano de montaña.

Comentarios: Autores previos consideraban a esta especie como una población de $O$. guatemalensis (ver Moctezuma y Halffter 2021).

95. O. pseudoundulans Zunino y Halffter, 1988 GRO.

Onthophagus pseudoundulans Zunino y Halffter, 1988a: 148; Zunino 2003; Krajcik 2006; Deloya y Covarrubias-Melgar 2014; Arriaga-Jiménez et al. 2016; Gasca-Álvarez et al. 2018; Escobar-Hernández et al. 2019; Halffter et al. 2019.

Patrón de distribución: Paleoamericano de montaña.

Comentarios: Autores previos consideraban que esta especie tenía dos subespecies: pseudoundulans y howdeni. Estos taxa son considerados como especies válidas en la actualidad (ver Halffter $e t$ al. 2019).

96. O. retusus Harold, 1869 HGO, OAX, PUE, VER.

Onthophagus retusus Harold, 1869: 108 (Figura 15); Zunino y Halffter 1988a; Deloya 1992b; Martín-Piera y Lobo 1993; Halffter et al. 1995; 2019; Arellano y Halffter 2003; Krajcik 2006; Pulido-Herrera y Zunino 2007; Ramírez-Ponce et al. 2009; 2019; Deloya-López 2011; Favila 2012; Alvarado et al. 2014; Moctezuma et al. 2016a; Arriaga-Jiménez et al. 2018; Kohlmann 
et al. 2018; Escobar-Hernández et al. 2019; Joaqui et al. 2019; Moctezuma y Halffter 2020b; Joaqui et al. 2021; Salomão et al. 2021.

Patrón de distribución: Paleoamericano de montaña.

Comentarios: Autores previos citaron a esta especie como $O$. chevrolati y $O$. chevrolati retusus (ver Halffter et al. 2019; Moctezuma y Halffter 2020b).

97. O. reyesi Zunino y Halffter, 1988 GTO, MICH.

Onthophagus reyesi Zunino y Halffter, 1988a: 146; Delgado 1997; Zunino 2003; Krajcik 2006; Pulido-Herrera y Zunino 2007; Arriaga-Jiménez et al. 2016; Deloya et al. 2016; Gasca-Álvarez et al. 2018; Halffter et al. 2019.

Patrón de distribución: Paleoamericano de montaña. 98. O. rhinolophus Harold, 1869

CAM, CHIS, OAX, PUE, TAB, VER; Guatemala, Belice, Honduras, Panamá, Colombia, Venezuela, Ecuador.

Onthophagus rhinolophus Harold, 1869: 510 (Figura 14); Boucomont y Gillet 1927; Boucomont 1932; Blackwelder 1944; Pereira y Halffter 1961; Halffter y Matthews 1966; Morón 1979; 1985; 1987; 2004; Morón et al. 1985; 2013; Delgado y Deloya 1990; Deloya 1992b; Halffter et al. 1992; 2007; Estrada et al. 1993; 1998; Thomas 1993; Morón y Blackaller 1997; Zunino y Halffter 1997; Delgado 1995; Delgado y Pensado 1998; Deloya y Morón 1998; Martínez y Cruz 1999; Palacios-Ríos et al. 1990; Estrada y Coates-Estrada 2002; Carrillo-Ruíz y Morón 2003; Zunino 2003; Pineda et al. 2005; Krajcik 2006; Delgado et al. 2006; 2012a; 2012b; Deloya et al. 2007; Padilla-Hill y Halffter 2007; Pulido-Herrera y Zunino 2007; Navarrete y Halffter 2008; Halffter y Halffter 2009; Medina y Pulido 2009; Villamarín-Cortez 2010; Barragán et al. 2011; Creedy y Mann 2011; Deloya-López 2011; Inward et al. 2011; Deloya-López y López-Huicochea 2012; Sarges et al. 2012; Alvarado et al. 2014; Breeschoten et al. 2016; Huerta-Crespo y Cruz-Rosales 2016; Huerta et al. 2016; Morón y Arce-Pérez 2016; Sánchez-de-Jesús et al. 2016; Favila 2017; Halffter 2017; Halffter y Morrone 2017; González-Tokman et al. 2018; Pini1la-Buitrago et al. 2018; Salomão et al. 2018; 2020; Sánchez-Hernández et al. 2018; 2020; Capello y Halffter 2019; Arellano et al. 2020; Servín-Pastor et al. 2020; Joaqui et al. 2021; Moctezuma 2021.

Patrón de distribución: Paleoamericano tropical.

Comentarios: Esta especie requiere revisión y es necesario corroborar su presencia en Sudamérica. Alvarado et al. (2014) utilizaron la grafía posterior incorrecta "rhynolophus" para referirse a esta especie.

99. O. rostratus Harold, 1869

EDOMEX, GRO, ¿HGO?, MOR, PUE.
Onthophagus rostratus Harold, 1869: 511; Boucomont y Gillet 1927; Boucomont 1932; Blackwelder 1944; Kohlmann y Sánchez 1984; Deloya 1988; 1992a; Delgado y Deloya 1990; Zunino y Halffter 1997; Delgado 1995; 1999; Vaz-de-Mello et al. 1998; Navarrete-Heredia et al. 2001; Zunino 2003; Pulido-Herrera y Zunino 2007; Halffter et al. 2008; Halffter y Halffter 2009; Jiménez-Sánchez et al. 2009; Trevilla-Rebollar et al. 2010; Yanes-Gómez y Morón 2010; Deloya et al. 2013; Morón et al. 2013; Deloya y Covarrubias-Melgar 2014; Márquez et al. 2017; Zaragoza-Caballero et al. 2019.

Patrón de distribución: Paleoamericano tropical.

Comentarios: Esta especie requiere revisión taxonómica. Halffter et al. (2008) y Márquez et al. (2017) citaron a esta especie de Hidalgo, pero este registro necesita confirmación.

100.O. rufescens Bates, 1887 CHIS, DGO, EDOMEX, GRO, GTO, JAL, MOR, OAX, PUE.

Onthophagus rufescens Bates, 1887: 78; Boucomont y Gillet 1927; Boucomont 1932; Blackwelder 1944; Howden et al. 1956; Halffter y Matthews 1966; Edmonds y Halffter 1978; Zunino 1980; Deloya 1988; Paulian 1988; Deloya et al. 1993; Thomas 1993; Zunino y Halffter 1997; Vaz de Mello et al. 1998; Morón et al. 2000; 2013; Navarrete-Heredia et al. 2001; Zunino 2003; Morón 2004; Krajcik 2006; Pulido-Herrera y Zunino 2007; Jiménez-Sánchez et al. 2009; Yanes-Gómez y Morón 2010; Delgado et al. 2012b; Deloya y Covarrubias-Melgar 2014; Márquez et al. 2017; Sánchez-Hernández et al. 2017; Hernández y Navarrete-Heredia 2018; Pinilla-Buitrago et al. 2018; Arriaga-Jiménez et al. 2019; Zaragoza-Caballero et al. 2019.

Onthophagus jalapensis Balthasar, 1939: 46; Martínez 1947; Krajcik 2006; sin. Pulido-Herrera y Zunino 2007: 115; Delgado et al. 2012b.

Patrón de distribución: Paleoamericano del Altiplano Mexicano.

Comentarios: Esta especie requiere de revisión taxonómica, ya que es posible que se trate de un complejo de especies crípticas.

101.O. sancristobalensis Moctezuma y Halffter, 2020 CHIS.

Onthophagus sancristobalensis Moctezuma y Halffter, 2020c: 498 (Figura 16).

Patrón de distribución: Paleoamericano de montaña. 102.O. sanpabloetlorum Moctezuma y Halffter, 2020 OAX.

Onthophagus sanpabloetlorum Moctezuma y Halffter, 2020b: 255; Salomão et al. 2021.

Patrón de distribución: Paleoamericano de montaña.

Comentarios: Esta especie fue previamente confundida con $O$. chevrolati y $O$. retusus por Escobar-Hernández et al. (2019). En consecuencia, Moctezuma y Halffter (2020b) sugirieron que 
todos los especímenes estudiados por Escobar-Hernández et al. (2019) deberían ser revisados nuevamente (500 individuos de $O$. chevrolati y 933 individuos de $O$. retusus, la mayoría de ellos con determinaciones taxonómicas erróneas). Sin embargo, es posible concluir que Salomão et al. (2021) ignoraron la recomendación de Moctezuma y Halffter (2020b), y simplemente cambiaron los nombres específicos de $O$. retusus por $O$. sanpabloetlorum, y $O$. chevrolati por O. retusus; debido a que Salomão et al. (2021) citaron un total de 500 individuos de $O$. retusus y 924 individuos de $O$. sanpabloetlorum.

Salomão et al. (2021) sugirieron que el nombre de esta especie debería ser $O$. sanplabloetlarum: "Onthophagus sanpabloetlorum Moctezuma and Halffter, 2020 [sic; it should be Onthophagus sanpabloetlarum, as befits the Latin declension of a name ending in an "a", San Pablo Etla, in the plural form]". Sin embargo, Onthophagus sanpabloetlorum es una grafía original correcta de acuerdo con el artículo 32.5 del Código Internacional de Nomenclatura Zoológica (CINZ, 1999), ya que no hay evidencia de una errata inadvertida: "Si en la publicación original misma... hay una evidencia clara de una errata inadvertida, sea lapsus calami o una errata de copia o de imprenta, debe corregirse. La transcripción o latinización incorrectas o el uso de una vocal de conexión inadecuada no deben considerarse erratas inadvertidas". De acuerdo con el artículo 33.4 del Código Internacional de Nomenclatura Zoológica (CINZ, 1999), O. sanpabloetlarum (Salomão et al. 2021) es considerada como una grafía posterior incorrecta, y por lo tanto es un nombre no disponible: "Grafías posteriores incorrectas. Cualquier grafía posterior de un nombre distinta a la grafía original correcta y que no sea un cambio obligatorio o una enmienda, es una «grafía posterior incorrecta»; no es un nombre disponible y, como grafía original incorrecta [Art. 32.4], no entra en homonimia y no puede usarse como un nombre de sustitución...". En consecuencia, se recomienda evitar el uso de la grafía $O$. sanpabloetlarum para referirse o citar a esta especie en futuros estudios para evitar confusiones.

103.O. santamariensis Moctezuma, Sánchez-Huerta y

Halffter, 2020

OAX.

Onthophagus santamariensis Moctezuma et al., 2020: 284 (Figura 17); Moctezuma 2021.

Patrón de distribución: Paleoamericano tropical.

Comentarios: Esta especie probablemente se encuentra localizada en el extremo sur del estado de Veracruz, ya que fue descrita cerca de la frontera con Oaxaca, en la región del Uxpanapa. Moctezuma et al. (2020) incorrectamente clasificaron a esta especie dentro del patrón de distri- bución neotropical típico.

104.O. schaefferi Howden y Cartwright, 1963

VER; Estados Unidos.

Onthophagus schaefferi Howden y Cartwright, 1963: 88; Halffter y Matthews 1966; Howden y Scholtz 1986; Deloya 1992b; Zunino y Halffter 1997; Halffter y Arellano 2002; Zunino 2003; Morón 2004; Krajcik 2006; Deloya et al. 2007; Escobar et al. 2007; Pulido-Herrera y Zunino 2007; Mora-Aguilar y Montes de Oca 2009; Deloya-López 2011; Márquez et al. 2017; Pinilla-Buitrago et al. 2018; Arriaga-Jiménez et al. 2019; Bezanson y Floate 2019; Rivera-Gasperín y Escobar-Hernández 2020.

Patrón de distribución: Paleoamericano tropical.

Comentarios: Esta especie requiere de revisión taxonómica, ya que es posible que se trate de un complejo de especies crípticas.

105.O. semiopacus Harold, 1869 GRO, OAX.

Onthophagus semiopacus Harold, 1869: 509; Boucomont y Gillet 1927; Boucomont 1932; Blackwelder 1944; Zunino y Halffter 1988a; Delgado 1997; Zunino 2003; Krajcik 2006; Pulido-Herrera y Zunino 2007; Deloya y Covarrubias-Melgar 2014; Moctezuma et al. 2016a; Halffter et al. 2019; Joaqui et al. 2019; Moctezuma y Halffter 2019a; 2020b; 2020c; Morrone 2020.

Patrón de distribución: Paleoamericano de montaña. Comentarios: Verdú et al. (2007), Halffter et al. (2012) y Márquez et al. (2017) citaron erróneamente a esta especie de Hidalgo. Es posible que estos registros correspondan a $O$. hidalgos u $O$. potosinus.

106.O. sharpi Harold, 1875

CHIS; Belice, Guatemala, Nicaragua, Costa Rica, Panamá, Colombia, Ecuador.

Onthophagus sharpi Harold, 1875: 138; Boucomont y Gillet 1927; Boucomont 1932; Blackwelder 1944; Goidanich y Malan 1964; Halffter y Matthews 1966; Howden y Young 1981; Young 1981; Gill 1991; Delgado y Deloya 1990; Hanski y Cambefort 1991; Thomas 1993; Delgado 1995; Zunino y Halffter 1997; Emlen 2000; 2001; Escobar 2000; Kohlmann y Solís 2001; Medina et al. 2001; Maes 1999; Zunino 2003; Krajcik 2006; Kohlmann et al. 2007; Noriega et al. 2007; Pulido-Herrera y Zunino 2007; Tarasov y Solodovnikov 2011; Solís y Kohlmann 2012; Latha et al. 2016; Chamorro et al. 2018; 2019; Sánchez-Hernández et al. 2020; Schwery y O'Meara 2021.

Patrón de distribución: Paleoamericano tropical.

Comentarios: Esta especie requiere de revisión taxonómica, ya que es posible que se trate de un complejo de especies crípticas.

107.O. skelleyi Sánchez-Huerta, Zunino y Halffter, 2018

QRO. 
Onthophagus skelleyi Sánchez-Huerta et al., 2018: 408; Halffter et al. 2019; Morrone 2020.

Patrón de distribución: Paleoamericano de montaña. 108.O. subcancer Howden, 1973

OAX, PUE, VER.

Onthophagus subcancer Howden, 1973: 335; Delgado y Deloya 1990; Zunino y Halffter 1997; Zunino 2003; Pineda et al. 2005; Krajcik 2006; Deloya et al. 2007; Halffter et al. 2007; Pulido-Herrera y Zunino 2007; Deloya-López 2011; Rös et al. 2012; Alvarado et al. 2014; Pinilla-Buitrago et al. 2018; Delgado y Mora-Aguilar 2019; Escobar-Hernández et al. 2019; Moctezuma y Halffter 2019b; Salomão et al. 2021.

Patrón de distribución: Paleoamericano mesoamericano.

109.O. subopacus Robinson, 1940

DGO, JAL, SON; Estados Unidos.

Onthophagus subopacus Robinson, 1940: 142; Howden y Cartwright 1963; Delgado 1997; Zunino y Halffter 1997; Navarrete-Heredia et al. 2001; Zunino 2003; Howden y Génier 2004; Krajcik 2006; Arriaga-Jiménez et al. 2019; Bezanson y Floate 2019.

Patrón de distribución: Paleoamericano de montaña.

110.O. tarascus Zunino y Halffter, 1988 $\mathrm{MICH}$.

Onthophagus tarascus Zunino y Halffter, 1988a: 153; Zunino 2003; Krajcik 2006; Pulido-Herrera y Zunino 2007; Arriaga-Jiménez et al. 2016; Deloya et al. 2016; Halffter et al. 2019.

Patrón de distribución: Paleoamericano de montaña.

Comentarios: Autores previos consideraban que esta especie tenía dos subespecies: tarascus y jaliscensis. Estos taxa son considerados como especies validas en la actualidad (ver Halffter et al. 2019).

111.O. totonicapamus Bates, 1887

CHIS; Guatemala.

Onthophagus totonicapamus Bates, 1887: 80; Boucomont y Gillet 1927; Boucomont 1932; Blackwelder 1944; Zunino y Halffter 1988a; Zunino 2003; Krajcik 2006; Pulido-Herrera y Zunino 2007; Halffter et al. 2019; Moctezuma y Halffter 2020a.

Patrón de distribución: Paleoamericano de montaña.

112.O. totonacus Moctezuma y Halffter, 2021 VER.

Onthophagus totonacus Moctezuma y Halffter, 2021 (Figura 18).

Patrón de distribución: Paleoamericano tropical.

Comentarios: Esta especie fue confundida con $O$. eulophus por autores previos (ver Moctezuma y Halffter 2021).

113.O. undulans Zunino y Halffter, 1988 GRO.

Onthophagus undulans Zunino y Halffter, 1988a: 136; Boucomont y Gillet 1927; Boucomont 1932; Blackwelder 1944; Anduaga 2000; Zuni- no 2003; Pulido-Herrera y Zunino 2007; Deloya y Covarrubias-Melgar 2014; Escobar-Hernández et al. 2019; Halffter et al. 2019.

Patrón de distribución: Paleoamericano de montaña.

Comentarios: Autores previos consideraban que esta especie tenía dos subespecies: undulans y oaxacanus. Ambos taxa son considerados como especies validas en la actualidad (ver Halffter et al. 2019).

114.O. veracruzensis Delgado y Pensado, 1988 CHIS, VER.

Onthophagus veracruzensis Delgado y Pensado, 1988: 76; Krajcik 2006; Delgado et al. 2006; Pulido-Herrera y Zunino 2007; Deloya-López 2011; Rivera et al. 2020.

Patrón de distribución: Paleoamericano tropical.

Comentarios: Deloya-López (2011) utilizó la grafía posterior incorrecta "veracruzcensis".

115.O. vespertilio Howden, Cartwright y Halffter, 1956

GRO.

Onthophagus vespertilio Howden et al., 1956: 2; Halffter y Matthews 1966; Zunino 1980; Redde11 1981; Paulian 1988; Zunino y Halffter 1988a; 1988b; 2007; Zunino 2003; Morón 2004; Hoffmann et al. 2004; Krajcik 2006; López-Guerrero y Zunino 2007; Pulido-Herrera y Zunino 2007; Jiménez-Sánchez et al. 2009; Slay et al. 2012; Deloya y Covarrubias-Melgar 2014; Márquez et al. 2017; Halffter et al. 2019.

Patrón de distribución: Paleoamericano de montaña.

Comentarios: Jiménez-Sánchez et al. (2009) y Deloya y Covarrubias-Melgar (2014) citaron erróneamente a esta especie para el Estado de México.

116.O. villanuevai Delgado y Deloya, 1990

GRO, OAX.

Onthophagus villanuevai Delgado y Deloya, 1990: 212 (Figura 19); Morón 1993; Delgado 1995; 1997; Navarrete-Heredia y Galindo-Miranda 1997; Zunino y Halffter 1997; Anduaga 2000; Zunino 2003; Krajcik 2006; Pulido-Herrera y Zunino 2007; Halffter y Halffter 2009; Deloya et al. 2013; Deloya y Covarrubias-Melgar 2014; Zaragoza-Caballero y Pérez-Hernández 2017.

Patrón de distribución: Paleoamericano tropical.

117.O. violetae Zunino y Halffter, 1997

¿CHIS?, VER.

Onthophagus violetae Zunino y Halffter, 1997: 174; Zunino 2003; Krajcik 2006; Deloya et al. 2007; Pulido-Herrera y Zunino 2007; Barragán et al. 2011; Deloya-López 2011; Arellano et al. 2013; Salomão et al. 2020; Sánchez-Hernández et al. 2020.

Patrón de distribución: Paleoamericano tropical.

Comentarios: Esta especie requiere de revisión taxonómica, ya que es posible que se trate de un complejo de especies crípticas. Arellano et al. (2013) y Sánchez-Hernández et al. (2020) citaron a esta especie de Chiapas. Sin embargo, este 
registro es dudoso ya que $O$. violetae parece ser un endémico de los bosques tropicales húmedos de Los Tuxtlas, Veracruz, mientras que Arellano et al. (2013) estudiaron una comunidad de bosque tropical seco de Chiapas.

118.O. viridichevrolati Moctezuma y Halffter, 2020 JAL.

Onthophagus viridichevrolati Moctezuma y Halffter, 2020b: 258.

Patrón de distribución: Paleoamericano de montaña.

Comentarios: Autores previos consideraban a esta especie como una población de $O$. chevrolati (ver Moctezuma y Halffter 2020b).

119.O. yucatanus Delgado, Peraza y Deloya, 2006

CAM, CHIS, QR, YUC; Belice, Guatemala.

Onthophagus yucatanus Delgado et al., 2006: 6 (Figura 20); Pulido-Herrera y Zunino 2007; Navarrete y Halffter 2008; Navarrete-Gutiérrez y Halffter 2008; Barragán et al. 2011; Latha et al. 2016; Sánchez-de-Jesús et al. 2016; Sánchez-Hernández et al. 2018; 2020; Capello y Halffter 2019; Santos-Heredia et al. 2019; Moctezuma et al. 2020; Rivera et al. 2020.

Patrón de distribución: Paleoamericano tropical. 120.O. zapotecus Zunino y Halffter, 1988

OAX.

Onthophagus zapotecus Zunino y Halffter, 1988c: 137; Pulido-Herrera y Zunino 2007; Gasca-Álvarez et al. 2018; Kohlmann et al. 2018; Escobar-Hernández et al. 2019; Arriaga-Jiménez et al. 2019; Salomão et al. 2021.

Patrón de distribución: Paleoamericano de montaña.

\section{Registros dudosos y erróneos}

1. O. atrosericeus Boucomont, 1932

Diversos autores sugieren que esta especie se encuentra presente en México, particularmente en el estado de Chiapas (Halffter et al. 1992; Pulido-Herrera y Zunino 2007; Sánchez-Hernández et al. 2017). Sin embargo, Sánchez-Hernández et al. (2020) sugieren que la cita de esta especie para México es errónea.

2. O. cavernicollis Howden y Cartwright, 1963

Pulido-Herrera y Zunino (2007) sugieren que esta especie se encuentra en México. Sin embargo, en la literatura citada por ellos no se ha encontrado alguna referencia que indique que la especie en cuestión se presenta en tal país. Por otra parte, Slay et al. (2012) indicaron que esta especie se encuentra únicamente en Estados Unidos.

3. O. curvicornis Latreille, 1811

Zunino (2003), Pulido-Herrera y Zunino (2007), Deloya-López (2011) y Morón et al. (2013) sugieren que esta especie se localiza en México, particularmente en la Ciudad de México, Hidalgo, Puebla y Veracruz. Sin embargo, Chamorro et al. (2019) precisaron que la distribución de $O$. curvicornis se restringe a Colombia, Ecuador y Venezuela.

4. O. dicranius Bates, 1887

Pulido-Herrera y Zunino (2007) y Deloya-López (2011) citaron erróneamente a esta especie para México. Estos registros podrían corresponder a $O$. petenensis u $O$. chinantecus.

5. O. nyctopus Bates, 1887

Rossini et al. (2018b) sugieren que la presencia de esta especie en México es dudosa y necesita confirmación.

6. O. praecellens Bates, 1887

Thomas (1993) y Jiménez-Sánchez et al. (2009) citaron erróneamente a esta especie para los estados de Chiapas y México, respectivamente.

7. O. rhinophyllus Harold, 1868

Pulido-Herrera y Zunino (2007) y Pinilla-Buitrago et al. (2018) citaron erróneamente a esta especie para México. Zunino (1981) indicó que los registros de $O$. rhinophyllus de México corresponden a $O$. maya, mientras que $O$. rhinophyllus se encuentra distribuido en Colombia y Venezuela (Delgado et al. 2006; Sánchez-Hernández et al. 2020).

8. O. subtropicus Howden y Cartwright, 1963

Halffter et al. (2008) y Márquez et al. (2017) citaron a esta especie para Hidalgo. Sin embargo, este registro se necesita verificar, ya que es un registro atípico dentro del área de distribución de esta especie, que se conoce únicamente de Texas, Estados Unidos (Howden y Cartwright 1963; Zunino y Halffter 1988a).

\section{Onthophagus mesoamericanus Zunino y Halffter, 1988 especie valida}

Con el motivo de elaborar la presente lista actualizada de los Onthophagus de México e incluir los sinónimos de cada especie, se revisó el caso de Onthophagus mesoamericanus Zunino y Halffter, 1988 (Figuras 25-27). Esta especie fue descrita originalmente por Zunino y Halffter (1988a: 123), quienes se basaron en tres especímenes provenientes de Costa Rica. De acuerdo con estos autores, el holotipo es un macho major (Figuras 25-27) que fue depositado en la Colección Personal del Dr. Gonzalo Halffter (examinado), mientras que los paratipos se encontraban en el Muséum National d'Histoire Naturelle de París (no examinados).

Posteriormente, Kohlmann y Solís (2001: 196) propusieron a $O$. mesoamericanus (Figuras 25-27) como un sinónimo subjetivo más moderno de $O$. cyanellus (Figuras 21-24). De acuerdo con estos autores, los paratipos de $O$. mesoamericanus, el lectotipo de O. cyanellus (Figura 21), y especímenes adicionales provenientes de Costa Rica fueron revisados. Kohlmann y Solís (2001) sugirieron que los caracteres morfológicos que distinguen a $O$. mesoamericanus (como son la coloración verde serícea, el margen del clípeo y declive pronotal casi negros, y la quilla frontal del macho inerme; Figura 25), son parte de la variación intrapoblacional de $O$. cyanellus (Figuras 21-22). Sin embargo, estos autores no proporcionaron ilustraciones de la variabilidad de la especie para soportar su propuesta. Adicionalmente, Kohlmann y Solís (2001) omitieron la revisión del holotipo de O. mesoamericanus (Figura 25) y la examinación de las estructuras genitales (Figuras 26-27); así como omitieron la revisión de la genitalia de $O$. cyanellus (Figuras 23-24). 
De forma subsecuente, Pulido-Herrera y Zunino (2007) incluyeron a O. mesoamericanus (Figura 25) como especie válida en su catálogo de los Onthophagini de América, pero no proporcionaron información para justificar el por qué desconocen la sinonimia previamente propuesta por Kohlmann y Solís (2001). Posteriormente, Solís y Kohlmann (2012) comentaron que revisaron nuevamente el material proveniente de la región de Cedros, Costa Rica, donde se localiza la localidad tipo de O. mesoamericanus (Figura 25). Estos autores sugirieron reiteradamente que la sinonimia de $O$. mesoamericanus (Figura 25) y O. cyanellus (Figuras 21-22) debería ser válida. Sin embargo, Solís y Kohlmann (2012) omitieron nuevamente la revisión del holotipo de $O$. mesoamericanus (Figura 25) y el estudio de material de $O$. cyanellus proveniente de toda su área de distribución. Por otra parte, Solís y Kohlmann (2012) prescindieron nuevamente de la presentación de material gráfico que soportara su decisión y de la revisión de la genitalia (Figuras 23-24, 26-27) de las especies en cuestión.

De acuerdo con el artículo 72.10 del Código Internacional de Nomenclatura Zoologica (CINZ, 1999), los tipos portanombre son los modelos internacionales de referencia que proporcionan objetividad a la nomenclatura zoológica. En consecuencia, los estudios de Kohlmann y Solís (2001) y Solís y Kohlmann (2012) carecen de una pieza clave, como lo es el estudio del holotipo de $O$. mesoamericanus (Figura 25). Para el presente estudio el holotipo de O. mesoamericanus fue examinado (Figura 25), y el lectotipo de O. cyanellus (Figura 21) fue revisado mediante registro fotográfico. Adicionalmente, fue examinado el registro fotográfico disponible en Bold Systems de dos hembras de O. mesoamericanus (ASSCR1194-11 y ASSCR1192-11; Barcode Index Number Registry For BOLD: ABA7531); así como fueron revisados distintos especímenes machos (Figura 22) y hembras de $O$. cyanellus provenientes de Chiapas (20 especímenes), Oaxaca (300 especímenes), Veracruz (50 especímenes), Puebla (20 especímenes), Costa Rica (20 especímenes) y Panamá (10 especímenes). Estos especímenes se encuentran resguardados en la Colección Entomológica “Dr. Miguel Ángel Morón Ríos” del Instituto de Ecología A.C., y en las colecciones personales del Dr. Gonzalo Halffter, del M. en C. José Luis Sánchez-Huerta y del autor del presente estudio.

De acuerdo con los especímenes revisados, ningún ejemplar de $O$. cyanellus (Figuras 21-22) mostró los caracteres que distinguen al holotipo de $O$. mesoamericanus (Figura 25), como son la coloración verde serícea, y el margen del clípeo y el declive pronotal casi negros. Algunos machos minor de $O$. cyanellus muestran la quilla frontal inerme. Sin embargo, todos los machos major que de $O$. cyanellus fueron examinados presentaron dos tubérculos triangulares posicionados en los extremos laterales de la quilla frontal (Figuras 21-22). Los caracteres diagnósticos externos de los machos minor de $O$. cyanellus y especies relacionadas usualmente se encuentran fuertemente reducidos, por lo que no se recomienda su consideración para delimitar especies o tomar decisiones taxonómicas y nomenclaturales (Zunino y Halffter 1988a; Arriaga-Jiménez et al. 2016; Moctezuma et al. 2016; Joaqui et al. 2019;
Moctezuma y Halffter 2020a; 2020b; 2020c). La coloración de los ejemplares de $O$. cyanellus que fue típicamente azul obscuro (Figuras 21-22) en los especímenes examinados, y nunca mostró las tonalidades de color verde seríceo típicas de O. mesoamericanus (Figura 25).

Además de los caracteres que fueron propuestos originalmente para separar a $O$. cyanellus y $O$. mesoamericanus (Zunino y Halffter 1988a), se proponen los siguientes caracteres externos de los machos major: Los tubérculos secundarios del pronoto están más fuertemente desarrollados en O. cyanellus (Figuras 21-22) que en O. mesoamericanus (Figura 25). El borde frontal de la proyección pronotal de O. cyanellus es casi recto o convexo (Figuras 21-22), mientras que en $O$. mesoamericanus muestra una concavidad anterior o incluso dos tubérculos frontales (Figura 25). La proyección pronotal de $O$. mesoamericanus (Figura 25) es más robusta que la de $O$. cyanellus (Figuras 21-22). Finalmente, la carina frontal está más fuertemente sinuada en la porción media de $O$. mesoamericanus (Figura 25) que en $O$. cyanellus (Figuras 21-22).

Adicionalmente, los siguientes caracteres genitales masculinos se sugieren para diagnosticar a las dos especies: El ángulo ventral de los parámeros de $O$. mesoamericanus es ligeramente agudo (Figura 26), mientras que en $O$. cyanellus es casi recto (Figura 23). El ápice de los parámeros es distintamente trapezoidal en O. mesoamericanus (Figura 26), mientras que la misma estructura es convexa pero no trapezoidal en $O$. cyanellus (Figura 23). Los dientes apicales de los parámeros son menos robustos en $O$. mesoamericanus (Figura 26).

Halffter et al. (2019) comentaron que el endofalito copulador del holotipo de $O$. mesoamericanus fue roto durante la disección y preparación original del espécimen (Figura 27), lo que evito temporalmente su examinación. Durante la examinación del holotipo anteriormente mencionado para el presente estudio se corroboró que el endofalito copulador se encuentra parcialmente roto en el lóbulo derecho (señalado con la elipse azul en la Figura 27). Sin embargo, esta estructura carece de valor taxonómico para $O$. cyanellus y sus especies relacionadas. Por otra parte, se preservó la integridad de los caracteres morfológicos de la quilla media, del lóbulo izquierdo, y del endofalito adicional medio. Se considera que estas estructuras son importantes para la determinación taxonómica y delimitación de especies de Onthophagus del continente americano (Zunino y Halffter 1988a; Howden y Gill 1993; Génier y Howden 1999; Arriaga et al. 2016; Moctezuma et al. 2016; Génier 2017; Rossini et al. 2018a; 2018b; Joaqui et al. 2019; Moctezuma y Halffter 2019a; 2019b; 2020a; 2020b; 2020c; 2021).

De esta forma, el lóbulo inferior izquierdo del endofalito copulador se encuentra pobremente desarrollado en $O$. mesoamericanus (Figura 27), mientras que esta estructura se encuentra fuertemente desarrollada en $O$. cyanellus (Figura 24); la quilla media del endofalito copulador de $O$. mesoamericanus se encuentra pobremente desarrollada y muestra únicamente un pliegue (Figura 27), mientras que la quilla media de $O$. cyanellus se encuentra fuertemente desarrollada y muestra dos pliegues distintos (Figura 24). Finalmente, el endofalito adicional medio de $O$. mesoame- 
ricanus muestra una forma obtusamente cuadrada (Figura 27); mientras que esta estructura tiene una fuerte concavidad media en $O$. cyanellus, lo que le confiere una forma de herradura (Figura 24).

Tomando en cuenta las consideraciones expuestas en los párrafos anteriores, y los caracteres morfológicos provenientes de la gran cantidad de ejemplares examinados en la presente contribución, se considera que existe suficiente evidencia para resucitar a $O$. mesoamericanus de la sinonimia previamente propuesta por Kohlmann y Solís (2001) y Solís y Kohlmann (2012). Esta decisión está basada principalmente en las diferencias presentes en el endofalito copulador y en el endofalito adicional medio (Figuras 24 y 27). Si bien, es posible que los caracteres diagnósticos externos puedan prestarse a confusión en especies cercanamente relacionadas del género Onthophagus, los caracteres de la genitalia suelen ser decisivos en la corroboración de la determinación taxonómica de sus especies; así como, en el descubrimiento de nuevos taxa (Zunino y Halffter 1988a; Howden y Gill 1993; Génier y Howden 1999; Arriaga-Jiménez et al. 2016; Moctezuma et al. 2016; Génier 2017; Rossini et al. 2018a; 2018b; Joaqui et al. 2019; Moctezuma y Halffter 2020a; 2020b; 2020c).

A pesar de que $O$. cyanellus y $O$. mesoamericanus podrían tener un área de simpatría en Costa Rica (Kohlmann y Solís 2001), las diferencias observadas en el edeago y los endofalitos (Figuras 23-24, 26-27) sugieren que podría existir un aislamiento reproductivo entre ambas especies. Estas estructuras morfológicas están relacionadas con la elaboración del espermatóforo y su depósito en el interior de la hembra, son heredadas como unidades funcionales, y son objeto de selección sexual postcopulatoria ya que su alineamiento correcto permite la inseminación (Werner y Simmons 2008). Por otra parte, se ha demostrado que la morfología genital de los Onthophagus tiene una fuerte señal filogenética (Tarasov y Solodovnikov 2011; Tarasov y Dimitrov 2016); así como, la genitalia de Onthophagus muestra niveles elevados de variación interespecífica $(\mathrm{Zu}-$ nino y Halffter 1988a; Howden y Gill 1993; Joaqui et al. 2019; Moctezuma y Halffter 2020a; 2020b; 2021; Moretto y Génier 2020).

\section{DISCUSIÓN \\ Compilación del listado de especies}

Gracias a la presente contribución, fue posible sintetizar el conocimiento actual sobre las especies del género $O n$ thophagus reportadas para México. Este listado actualizado toma en consideración los numerosos descubrimientos y cambios propuestos en la literatura reciente; así como, cubre la mayor parte de la literatura relevante sobre el género en México y otros países, incluyendo numerosos estudios taxonómicos y ecológicos. Y es aquí donde radica la principal limitación de este estudio, ya que se basó únicamente en literatura publicada y se evitó deliberadamente la consulta de bases de datos disponibles, como son Global Biodiversity Information Facility y Escobar et al. (2015). Se tomó esta decisión debido a que las bases de datos anteriormente mencionadas presentan numerosos problemas; como son los errores de determinación taxonómica, errores en las localidades de distribución de las especies, y una falta de actualización en los taxa reconocidos actualmente. En consecuencia, estas bases de datos requieren de un importante proceso de depuración y revisión por parte de taxónomos expertos antes de ser utilizadas de forma confiable. Estos problemas no son únicos del género Onthophagus, sino que también se extienden a otros géneros de escarabajos del estiércol como Phanaeus MacLeay, 1819 (Lizardo et al. 2017), y probablemente a toda la subfamilia Scarabaeinae.

\section{Riqueza de especies de México}

Actualmente se considera que existen unas 193 especies descritas de Onthophagus en América (Moctezuma y Halffter 2021; presente estudio). México muestra una riqueza de especies realmente notable con 120 especies, albergando a aproximadamente el $62 \%$ de los Onthophagus del continente y superando por mucho a otros grandes países americanos, tales como Canadá, Estados Unidos, Colombia, Ecuador, Perú y Brasil (cuadro 1). Tomando en cuenta el estado actual del conocimiento, es posible concluir que México es el principal centro de diversificación del género Onthophagus en el Nuevo Mundo. Aunque se tiene conocimiento de que existen al menos 22 especies sin describir en América (Creedy y Mann 2011; Rossini et al. 2018b; Moctezuma 2021), este hecho no cambiaría la preponderancia de la diversidad de Onthophagus de México. Por otra parte, en la actualidad se encuentran en proceso de descripción diversas especies que provienen de México, por lo que la riqueza de especies de este país seguirá incrementándose en años próximos.

Ha habido un incremento notable en el número de especies de México desde los estudios de Zunino (2003) y Pulido-Herrera y Zunino (2007), quienes reportaron 77 y 91 especies, respectivamente. Este incremento se puede asociar a diversas razones. Una de ellas, es el reconocimiento de numerosas especies crípticas dentro del conocido grupo de especies de $O$. chevrolati, especies que se encontraban relegadas al rango de subespecie, razón por la que usualmente pasaban desapercibidas a pesar de que en muchos casos representan endemismos muy distintivos de regiones montañosas aisladas de México (Halffter et al. 2019; Joaqui et al. 2019). Otra razón es la aparición de numerosas descripciones aisladas de especies (Arriaga-Jiménez et al. 2016; Moctezuma et al. 2016a; 2020; Delgado y Mora-Aguilar 2019; Moctezuma y Halffter 2019a; 2019b; 2020c); así como de estudios taxonómicos más completos (Arriaga-Jiménez et al. 2019; Joaqui et al. 2019; Moctezuma y Halffter 2020a; 2020b; 2021).

Algunos estudios ecológicos han tenido un papel importante en su aportación al incremento del conocimiento de los Onthophagus de México. Por ejemplo, el trabajo de Moctezuma et al. (2016b) en dos montañas de la región central de Puebla derivó en el descubrimiento de $O$. bolivari, $O$. clavijeroi y $O$. martinpierai. Otro caso es el estudio de Joaqui et al. (2021) en el Pico de Orizaba, que produjo el descubrimiento de $O$. orizabensis y $O$. totonacus. Finalmente, un caso realmente notable es el análisis de los Scarabaeinae de la región de Los Chimalapas en Oaxaca (Moctezuma 2021), 
que trajo a la luz al menos cuatro especies nuevas $(O$. chinantecus, $O$. istmenus, $O$. santamariensis y una especie relacionada con $O$. neomirabilis). A su vez, la examinación de los especímenes colectados para el estudio de los Chimalapas (Moctezuma 2021), así como su comparación con especímenes de otras regiones, tuvo como consecuencia el descubrimiento de $O$. sancristobalensis y $O$ pseudoguatemalensis. Algunos estudios ecológicos han representado aportaciones notables al conocimiento de algunos estados en particular, aunque no deriven en el descubrimiento de especies nuevas. Tal sería el caso de la región de Calakmul en Campeche analizada por Capello y Halffter (2019), o La Selva Lacandona en Chiapas (Navarrete y Halffter 2008; Sánchez-de-Jesús et al. 2016). Es importante considerar que no en todos los estados de la República Mexicana se ha realizado un esfuerzo de muestreo uniforme, pues existen numerosas regiones poco colectadas o inexploradas desde el punto de vista entomológico (Escobar et al. 2009). Futuros muestreos en áreas poco exploradas de México podrían derivar en el descubrimiento de nuevos taxa.

\section{Causas ecológicas y biogeográficas de la diversidad de Onthophagus en México}

La enorme diversidad de Onthophagus de México puede ser explicada por distintos factores. Uno de estos factores es que México es un mosaico biótico altamente heterogéneo (Morrone 2019), pues en su territorio se encuentran diversos tipos de vegetación, que van desde los desiertos, matorrales y pastizales, hasta bosques templados y tropicales (Rzedowski 2006). Recientemente, se ha sugerido que una elevada heterogeneidad ambiental puede relacionarse con una elevada riqueza de especies de los escarabajos del estiércol del neotrópico (Pessoa et al. 2020). Por otra parte, México es un país que posee numerosos sistemas montañosos, que presentan condiciones climáticas tanto tropicales como templadas (Mastretta-Yanes et al. 2015; Halffter y Morrone 2017; Morrone 2019; 2020). Estos sistemas montañosos representan un espacio geográfico de alta complejidad, que ha favorecido distintos procesos biogeográficos y evolutivos a lo largo de periodos geológicos anteriores; como son la dispersión, la fragmentación, el aislamiento y la especiación por vicarianza (Mastretta-Yanes et al. 2015; Halffter 2017; 2019; Halffter y Morrone 2017; Halffter et al. 2019; Morrone 2019; 2020). Estos procesos han promovido la aparición de un elevado número de endemismos adaptados exclusivamente a las zonas montañosas de México (Mastretta-Yanes 2015; Halffter y Morrone 2017; Halffter et al. 2019; Morrone 2020), como son la mayoría de las especies relacionadas con $O$. chevrolati (cuadro 2; Zunino y Halffter 1988a; Halffter et al. 2019; Joaqui et al. 2019; Moctezuma y Halffter 2020a; 2020b). El alto número de endemismos de montaña de Onthophagus promueve a su vez un elevado recambio de especies entre las distintas zonas montañosas de México (Moctezuma et al. 2018; Arriaga-Jiménez et al. 2018).

Por otra parte, México es parte de la Zona de Transición Mexicana, la que es considerada como un espacio geográfico donde interactúan y se sobrelapan biotas con historias biogeográficas y evolutivas distintas (Lobo y Halffter 2000;
Mastretta-Yanes et al. 2015; Halffter 2017; 2019; Halffter y Morrone 2017; Morrone 2019; 2020; Joaqui et al. 2021). En este aspecto, todos los Onthophagus de México y del Nuevo Mundo forman parte del patrón de distribución o cenocrón paleoamericano (Zunino y Halffter 1988a; Halffter y Morrone 2017; Halffter et al. 2019 Morrone 2020). A su vez, los Onthophagus se clasifican en los subpatrones paleoamericano de montaña, paleoamericano del Altiplano Mexicano, paleoamericano tropical, paleoamericano mesoamericano y paleoamericano bajacaliforniano (cuadro 2; Halffter y Morrone 2017; Halffter et al. 2019; Moctezuma y Halffter 2019a; Morrone 2020). Cada subpatrón paleoamericano incluye a especies que hipotéticamente evolucionaron bajo condiciones ecológicas y geográficas diferentes (Halffter y Morrone 2017; Halffter et al. 2019; Moctezuma y Halffter 2019a; Morrone 2020), e incluso en periodos geológicos distintos (Halffter 2017; 2019; Moctezuma y Halffter 2019a; Moctezuma et al. 2019; Morrone 2019; 2020).

\section{Distribución paleoamericana tropical vs distribución boreotropical en Onthophagus}

Recientemente, un grupo de autores (Kohlmann et al. 2019) sugirió que el concepto de distribución boreotropical debería sustituir al concepto de distribución paleoamericana tropical para referirse a algunas especies americanas del género Onthophagus, debido a que lo consideran como un concepto "mejor" fundamentado. La hipótesis de la distribución boreotropical sugiere que una franja continua de bosque boreotropical se extendió completamente sobre el hemisferio norte durante el Cenozoico temprano (Wolfe 1978; Sanmartín et al. 2001). Posteriormente, el cambio en las condiciones climáticas provocó la desaparición y retracción del bosque boreotropical, promoviendo el aislamiento y especiación por vicarianza de los organismos asociados con él (Wolfe 1978). Como consecuencia, existen en la actualidad grupos de organismos que tienen su centro de diversificación en las porciones tropicales de Norteamérica, aparecen en el registro fósil durante el Paleoceno temprano, y tienen a sus grupos hermanos en las regiones paleotropicales y especies derivadas en Sudamérica (Lavin y Luckow 1993).

Sin embargo, la hipótesis de distribución boreotropical no es apoyada por los conocimientos actuales que giran en torno a los Onthophagus de América. Kohlmann et al. (2019) soportaron sus conclusiones con el estudio de Howden y Gill (1993), e ignoraron las hipótesis filogenéticas más recientes (como Breeschoten et al., 2016). Howden y Gill (1993) sugirieron que los Onthophagus americanos provienen de al menos 20 ancestros distintos, contrario a las hipótesis filogenéticas más recientes que sugieren que este género conforma una unidad monofilética (Emlen et al. 2005; Breeschoten et al. 2016; Schwery y O'Meara 2021). Breeschoten et al. (2016) y Moctezuma et al. (2019) sugieren que el género Onthophagus apareció en América durante el Oligoceno, en un periodo muy posterior a la expansión y subsecuente retracción de los bosques boreotropicales (Wolfe 1978; Sanmartín et al. 2001). Asimismo, los grupos hermanos de los Onthophagus americanos se encuentran 
probablemente en Australia (Breeschoten et al. 2016) o en Asia (Schwery y O'Meara 2021), fuera de los paleotrópicos.

Por otra parte, diversas reconstrucciones paleoambientales sugieren que los bosques neotropicales húmedos de América aparecieron durante el Paleoceno-Oligoceno (Solórzano-Kraemer 2007; Jaramillo 2019; Carvalho et al. 2021), siendo este tipo de bosque el hábitat ideal para las especies paleoamericanas tropicales (Zunino y Halffter 1997), mientras que se ha sugerido que el ancestro de los Onthophagus americanos podría haber pertenecido al patrón paleoamericano tropical (Moctezuma et al. 2019). Además, no se conoce evidencia en el registro fósil para considerar que Onthophagus estuvo presente en América durante el Paleoceno temprano (Tarasov et al. 2016). Por último, el centro de diversificación de los Onthophagus paleoamericanos tropicales se encuentra en los bosques tropicales de Centroamérica y del norte de Sudamérica (Zunino y Halffter 1997; Kohlmann y Solís 2001); y no en Norteamérica tropical, como sugieren Kohlmann et al. (2019) y como sería de esperarse para un grupo boreotropical (Lavin y Luckow 1993). Tomando en cuenta los conocimientos actuales sobre los Onthophagus americanos, se puede concluir que no hay evidencias consistentes para considerarlos como un grupo boreotropical.

\section{Distribución estatal de la diversidad de Onthophagus de México}

Tomando en consideración la distribución estatal de la riqueza de especies de Onthophagus en la República Mexicana (cuadro 3), salta a primera vista que Oaxaca es el estado con mayor riqueza específica, con cerca del $38 \%$ de las especies del país. Este hecho sería de esperarse, ya que Oaxaca es considerado como el estado mexicano con mayor diversidad biológica para numerosos grupos de organismos (Peterson et al. 2003; García-Mendoza et al. 2004; Villaseñor y Ortiz 2014; Santos-Moreno et al. 2014). La diversidad de Onthophagus de Oaxaca es similar a la de Costa Rica, y mucho mayor que la países como Estados Unidos, Panamá, Colombia, Ecuador y Brasil.

El notable incremento de la diversidad de Onthophagus de Oaxaca en comparación con el estudio de Zunino (2003), quien reportó únicamente 28 especies, se debe a diversos factores. Uno de los factores es que se incrementó notablemente el número de descripciones de especies nuevas en años recientes (Arriaga-Jiménez et al. 2019; Delgado y Mora-Aguilar 2019; Moctezuma y Halffter 2019b; 2020b; Moctezuma et al. 2020). Por otra parte, ha incrementado el número de estudios faunísticos y ecológicos en Oaxaca, lo que ha conducido frecuentemente al descubrimiento de nuevos registros de especies y especies nuevas (Alvarado et al. 2014; Escobar-Hernández et al. 2019; Ramírez-Ponce et al. 2009; 2019; Moctezuma 2021). Debido a que diversos territorios y ecosistemas de Oaxaca permanecen inexplorados (Escobar et al. 2009), se esperaría que futuras prospecciones biológicas aporten numerosos descubrimientos adicionales.

Otros estados de gran relevancia por su número de especies son, en orden de importancia, Veracruz, Chiapas, y
Puebla, todos con 30 o más especies (cuadro 2). La riqueza de especies de estos estados es comparable con la de Estados Unidos (cuadro 1). Junto con Oaxaca, estos estados también son considerados dentro de los más biológicamente diversos de la República Mexicana (Deloya 1992b; 2011; Llorente-Bousquets y Ocegueda 2008; Morón et al. 2013; Villaseñor y Ortiz 2014; Sánchez-Hernández et al. 2020).

En el aspecto contrario, destaca particularmente el estado de Baja California, donde no se ha encontrado ningún registro de especies de Onthophagus en su territorio, cuando al menos debería presentarse $O$. cartwrighti (Moctezuma y Halffter 2021). De forma interesante, una gran parte del país permanece pobremente estudiada (Escobar et al. 2009), pues numerosos estados como Aguascalientes, Baja California Sur, Chihuahua, Coahuila, Nayarit, Sonora, Tabasco, Zacatecas, Sinaloa, Guanajuato, Nuevo León, Tlaxcala y Quintana Roo presentan cinco especies o menos, mientras que en otras entidades como la Ciudad de México, Colima, Querétaro, Tamaulipas y Yucatán se han reportado entre seis y ocho especies únicamente.

Debido a que la mayor parte de los territorios inexplorados de México desde el punto de vista entomológico se encuentran en los estados del norte del país, se esperaría que su riqueza de especies se incremente con forme aumente el número de estudios realizados en sus territorios (Escobar et al. 2009). Existen distintas especies presentes en los Estados Unidos cuya distribución parece estar artificialmente limitada por la frontera de este país con México, como serían los casos de $O$. arnetti Howden y Cartwright, 1963; O. knausi Brown, 1927; O. pennsylvanicus Melsheimer, 1806; O. subtropicus Howden y Cartwright, 1963; y O. velutinus Horn, 1875 (Howden y Cartwright 1963; Zunino y Halffter 1988a). Por otra parte, hay especies reportadas exclusivamente para Estados Unidos y que están asociadas a roedores como O. cynomysi Brown, 1927; cuyo hospedero se encuentra también en México (Moctezuma y Halffter 2021).

Además, se esperaría que estados tropicales como Quintana Roo y Yucatán tuvieran una riqueza de especies similar a la de Campeche. Zunino (2003) no reportó ninguna especie de Onthophagus para Campeche, pero el estudio de Capello y Halffter (2019) incrementó notablemente el conocimiento de su fauna de Onthophagus y de Scarabaeinae en general. Aunque Tabasco tiene un territorio reducido, se esperaría que compartiera diversas especies de Onthophagus con los estados de Veracruz, Oaxaca y Chiapas. Además, el estado de Nayarit podría albergar una diversidad similar a la de Jalisco y se esperaría que compartieran diversas especies.

\section{Endemismo}

De las 120 especies que se conocen para México, 85 son encontradas exclusivamente en este país. Es decir, más del $70 \%$ de los Onthophagus presentes en México son endémicos. Otro caso excepcional con un elevado número de endemismos es el de la fauna Australiana de Onthophagus, que presenta un porcentaje de endemismo mayor al 97\% (Matthews 1972). El caso Australiano se relaciona notablemente con el aislamiento que ha sufrido por su situación insular, y 
por su coevolución con una fauna de mamíferos dominada por marsupiales (Matthews 1972). Como se ha mencionado en párrafos anteriores, los sistemas montañosos han servido como un importante centro de aislamiento, diversificación y endemismo en el caso de México (Mastretta-Yanes et al. 2015; Halffter y Morrone 2017; Morrone 2020) lo que se ve evidenciado en el elevado número de especies paleoamericanas de montaña (cuadro 2).

En el caso de los estados de la República Mexicana, Oaxaca (15 especies) y Guerrero (9 especies) destacan por su elevado número de endemismos (cuadro 3). En estudios anteriores, se ha considerado al Eje Neovolcánico Transversal como el principal centro de diversificación de la fauna de Onthophagus de montaña (Zunino y Halffter 1988a; Halffter y Morrone 2017; Halffter et al. 2019). Sin embargo, los resultados del presente estudio evidencian que la Sierra Madre del Sur en los estados de Oaxaca y Guerrero y la Sierra Norte de Oaxaca también han sido importantes centros de diversificación para la fauna de Onthophagus de México. El elevado número de endemismos de la Sierra Madre del Sur y de la Sierra Norte había pasado desapercibido, debido a que numerosas especies de Guerrero y Oaxaca se encontraban relegadas al rango de subespecie (Zunino y Halffter 1988a), hecho que fue recientemente corregido por Halffter et al. (2019).

\section{AGRADECIMIENTOS}

La presente publicación está dedicada al Dr. Juan José Morrone, en honor a sus grandes contribuciones en el estudio de la biogeografía de Latinoamérica. Un agradecimiento especial al M. en C. José Luis Sánchez-Huerta por proveer distintas fotografías de especies mexicanas del género Onthophagus (O. halffteri, O. incensus, O. istmenus, $O$. chevrolati, O. moroni, O. pseudofuscus, O. rhinolophus y $O$. santamariensis); a Alfonso Aceves-Aparicio por las fotografías de $O$. lecontei y $O$. mexicanus; a Jiř́i Hájek (Národní Muzeum de Praga, República Checa) por las imágenes de O. nudifrons; al Dr. José Luis Navarrete-Heredia por su amable invitación para participar en el presente homenaje; y al Dr. Gonzalo Halffter por su amistad, asesoría y colaboración, que han derivado en la publicación reciente de distintos estudios sobre el género Onthophagus y en la revisión del holotipo de $O$. mesoamericanus. Finalmente, quisiera extender un agradecimiento a Mario Cupello (Universidade Federal do Paraná) por su amable apoyo para obtener las fotografías del lectotipo de $O$. cyanellus, actualmente depositado en el Natural History Museum de Londres.

\section{LITERATURA CITADA}

Alvarado, F., F. Escobar and J. Montero-Muñoz. 2014. Diversity and biogeographical makeup of the dung beetle communities inhabiting two mountains in the Mexican Transition Zone. Organisms Diversity and Evolution, (14): 105-114.

Alvarado, F., E.R. Andrade, B.A. Santos, G. Prescott, G. Souza and F. Escobar. 2018a. Forest cover is more important than farmland heterogeneity and livestock inten- sification for the retention of dung beetle phylogenetic diversity. Ecological Indicators, (93): 524-532.

Alvarado, F., F. Escobar, D.R. Williams, V. Arroyo-Rodríguez and F. Escobar-Hernández. 2018b. The role of livestock intensification and landscape structure in maintaining tropical Biodiversity. Journal of Applied Ecology, 55(1): 185-194.

Alvarado, F., R.P. Salomão, Á. Hernández-Rivera and A.F. de Araujo Lira. 2020. Different responses of dung beetle diversity and feeding guilds from natural and disturbed habitats across a subtropical elevational gradient. Acta Oecologica, (104): 103533.

Amell-Caez, Y., I. deCastro-Arrazola, H. García, J.D. Monroy-G. and J.A. Noriega. 2019. Spatial diversity of dung beetle assemblages (Coleoptera: Scarabaeidae: Scarabaeinae) in five ecoregions from Sucre, Colombian Caribbean coast. Revista Colombiana de Entomología, 45(2): e7963.

Andresen, S. 2008a. Dung beetle assemblages in primary forest and disturbed hábitats in a tropical dry forest landscape in western Mexico. Journal of Insect Conservation, (12): 639-650.

Andresen, S. 2008b. Short-term temporal variability in the abundance of tropical dung beetles. Insect Conservation and Diversity, 1(2): 120-124.

Anduaga, S. 2000. Escarabajos coprófagos (Coleoptera: Scarabaeoidea) asociados a hongos en la Sierra Madre Occidental, Durango, México con una compilación de las especies micetófagas. Acta Zoológica Mexicana (n.s.), (80): 119-130.

Anduaga, S. 2007. Nuevos registros de escarabajos coprófagos (Coleoptera: Scarabaeidae) en detritus de madrigueras de Neotoma albigula Hartley (Rodentia: Muridae). Acta Zoológica Mexicana (n.s.), 23(3): 143-144.

Anduaga, S. y G. Halffter. 1991. Escarabajos asociados a madrigueras de roedores (Coleoptera: Scarabaeidae: Scarabaeinae). Folia Entomológica Mexicana, (81): 186-197.

Aragón-García, A., A.M. Tapia-Rojas y B.C. Pérez-Torres. 2011. Insectos de Puebla. (pp. 142-148). En: Comisión Nacional para el Conocimiento y Uso de la Biodiversidad (Ed.). La biodiversidad en Puebla: Estudio de estado. Comisión Nacional para el Conocimiento y Uso de la Biodiversidad, Gobierno del Estado de Puebla, Benemérita Universidad Autónoma de Puebla, Ciudad de México, Puebla.

Arellano, L. and G. Halffter. 2003. Gamma diversity: derived from and a determinant of alpha diversity and beta diversity. An analysis of three tropical landscapes. Acta Zoológica Mexicana (n.s.), (90): 27-76.

Arellano, L. Y C. Castillo-Guevara. 2004. Efecto de los incendios forestales no controlados en el ensamble de escarabajos coprófagos (Coleoptera: Scarabaeidae) en un bosque templado del centro de México. Revista Mexicana de Biodiversidad, (85): 854-865.

Arellano, L., M. Favila and C. Huerta. 2004. Diversity of dung and carrion beetles in a disturbed Mexican tropical montane cloud forest and shade coffee plantations. Biodiversity and Conservation, (14): 601-615. 
Arellano, L., J.L. León-Cortés and G. Halffter. 2008. Response of dung beetle assemblages to Landscape structure in remnant natural and modified habitats in southern Mexico. Insect Conservation and Diversity, (1): 253-262.

Arellano, L., A.J. Martínez, E. Lezama-Delgado and M. Zunino. 2009. Dung beetles (Coleoptera: Scarabaeinae) in rabbit dung heaps: First report for Mesoamerica. The Coleopterists Bulletin, 63(1): 101-104.

Arellano, L., J.L. León-Cortés, G. Halffter and J. Montero. 2013. Acacia woodlots, cattle and dung beetles (Coleoptera: Scarabaeinae) in a Mexican silvopastoral landscape. Revista Mexicana de Biodiversidad, (84): 650660.

Arellano, L., M. Cruz-Rosales y C. Huerta. 2014. El estiércol, material de desecho, de provecho y algo más... Instituto de Ecología, A.C., Xalapa.

Arellano, L., C. Castillo-Guevara, C. Huerta, A. Germán-García and C. Lara. 2017. Nesting biology and life history of the dung beetle Onthophagus lecontei (Coleoptera: Scarabaeinae). Animal Biology, (67): 41-52.

Arellano, L., A. Díaz and F. Escobar-Hernández. 2020. XV. Escarabajos copronecrófagos. Insecta: Coleoptera: Scarabaeidae y Silphidae. (pp. 204-215). En: Samain, M.S. and G. Castillo- Campos (Eds.). Biodiversidad del Santuario del Bosque de Niebla, Xalapa, Veracruz. Instituto de Ecología, A.C, Xalapa.

Arias-Buriticá, J.A., P. Delgado-Gómez, F.A. González-A and F.Z. Vaz-de-Mello. 2011. New records of dung beetles (Coleoptera: Scarabaeidae: Scarabaeinae) for Chocó department (Colombia). Acta Zoológica Mexicana (n.s.), 27(3): 875-878.

Arriaga, A., G. Halffter and C. Moreno. 2012. Biogeographical affinities and species richness of copronecrophagous beetles (Scarabaeoidea) in the southeastern Mexican High Plateau. Revista Mexicana de Biodiversidad, 83: 519-529.

Arriaga-Jiménez, A., L. Roy, J.P. Lumaret and M. Bertrand. 2014. Phoretic Mexican macrochelids on high altitude ecosystems (Acari, Mesostigmata). Bulletin de la Société Entomologique de France, 119(3): 363-380.

Arriaga-Jiménez, A., V. Moctezuma, M. Rossini, M. Zunino and G. Halffter. 2016. A new species of Onthophagus (Scarabaeoidea: Scarabaeinae) from the Mexican Transition Zone, with remarks on its relationships and distribution. Zootaxa, 4072(1): 135-143.

Arriaga-Jiménez, A., M. Rös and G. Halffter. 2018. High variability of dung beetle diversity patterns at four mountains of the Trans-Mexican Volcanic Belt. PeerJ, (6): e4468.

Arriaga-Jiménez, A., F. Escobar-Hernánez, M. Rös and B. Kohlmann. 2019. The establishment of the Onthophagus anthracinus (Coleoptera: Scarabaeidae) species complex and the description of a new species. The $\mathrm{Ca}$ nadian Entomologist, 152(1): 1-17.

Balthasar, V. 1939. Neue Arten der palaearktischen und neotropischen coprophagen Scarabaeiden. Entomologické Listy (Folia Entomologica), (2): 41-47.

Barbero, E. 2001. Scarabaeidae (Coleoptera) copronecró- fagos interesantes del Departamento de Río San Juan, Nicaragua. Revista Nicaragüense de Entomología, (5558): 11-21.

Barragán, F., C.E. Moreno, F. Escobar, G. Halffter and D. Navarrete. 2011. Negative impacts of human land use on dung beetle functional diversity. PLOS ONE, 6(3): e17976.

Barragán, F., C.E. Moreno, F. Escobar, J. Bueno-Villegas and G. Halffter. 2014. The impact of grazing on dung beetle diversity depends on both biogeographical and ecological context. Journal of Biogeography, (41): 1991-2002.

Basto-Estrella, G.S., R.I. Rodríguez-Vivas, H. Delfín-González and E. Reyes-Novelo. 2013. Dung beetle (Coleoptera: Scarabaeinae) diversity and seasonality in response to use of macrocyclic lactones at cattle ranches in the mexican neotropics. Insect Conservation and Diversity, (7): 73-81.

Bates, H.W. 1887. Biologia Centrali-Americana. Insecta. Coleoptera. Vol. II. Part 2. Pectinicornia and Lamellicornia. Taylor and Francis, Londres.

Bezanson, G.A. and K.D. Floate. 2019. An updated checklist of the Coleoptera associated with livestogk dung on pastures in America north of Mexico. The Coleopterist Bulletin, 73(3): 655-683.

Blackwelder, R.E. 1944. Checklist of the coleopterous insects of Mexico, Central America, the West Indies and South America. Part 2. Bulletin of the United States National Museum, (185): 189-341.

Blanco-Casanova, J.L. 1988. Catálogo de los Scarabaeidae (Coleoptera) coprófagos y necrófagos del estado del Táchira, Venezuela. Parte II. Revista Cientifica Unet, 2(1): 39-48.

Boucomont, A. 1932. Synopsis des Onthophagus d'Amérique du Sud (Col. Scarab). Annales de la Société Entomologique de France, (101): 293-332.

Boucomont, A. et J.J.E. Gillet. 1927. Fam. Scarabaeidae. Subfam. Coprinae II. (pp. 103-263). En: Gillet J.J.E y A. Boucomont. Coleopterorum Catalogus. Scarabaeidae: Coprinae, Termitotroginae (Partes 38 et 90). W. Junk, Berlin.

Breeschoten, T., C. Doorenweerd, S. Tarasov and A.P. Vogler. 2016. Phylogenetics and biogeography of the dung beetle genus Onthophagus inferred from mitochondrial genomes. Molecular Phylogenetics and Evolution, (105): 86-95.

Brown, W.J. 1927. Four new species of Onthophagus (Coleoptera). The Canadian Entomologist, (59): 128-133.

Bustos, L. y A. Lopera. 2003. Preferencia por cebo de los escarabajos coprófagos (Coleoptera: Scarabaeidae: Scarabaeinae) de un remanente de bosque seco tropical al norte del Tolima (Colombia). (pp. 59-65). En: Onore, G., P. Reyes-Castillo y M. Zunino (Eds). Escarabeidos de Latinoamérica: estado del conocimiento. Monografías 3ercer Milenio M3M y Sociedad Entomológica Aragonesa, Zaragoza.

Cambefort, Y. 1991. Chapter 4. Biogeography and Evolution. (pp. 51-67). En: Hanski, I. and Y. Cambefort (Eds.). Dung beetle ecology. Princeton University 
Press, Princeton.

Campos, F. 1921. Estudios sobre la fauna entomológica del Ecuador. Revista del Colegio Nacional Vicente Rocafuerte, (6): 26-100.

Capello, V. y G. Halffter. 2019. Listado ilustrado de las especies de Scarabaeinae (Coleoptera: Scarabaeidae) de la Reserva de la Biósfera de Calakmul, Campeche, México. Dugesiana, 26(2): 103-131.

Carrillo-Ruíz, H. y M.A. Morón. 2003. Fauna de Coleoptera Scarabaeoidea de Cuetzalan del Progreso, Puebla, México. Acta Zoológica Mexicana (n.s.), (88): 87-121.

Carrillo-Ruíz, H., I. Guerra-González, M. Sánchez-Carri1lo, M.A. Morón y S.P. Rivas-Arancibia. 2017. Fauna de Scarabaeoidea (Insecta: Coleoptera) de Calmeca, Tepexco, Puebla, México. Acta Zoológica Mexicana (n.s.), 33(2): 251-265.

Carvajal, V., S. Villamarín y A.M. Ortega. 2011. Escarabajos del Ecuador. Principales géneros. Serie Entomología, Número. 1. Escuela Politécnica Nacional, Quito.

Carvalho, M.R., C. Jaramillo, F. de la Parra, D. Caballero-Rodríguez, F. Herrera, S. Wing, B.L. Turner, C. D’Apolito, M. Romero-Báez, P. Narváez, C. Martínez, M. Gutierrez, C. Labandeira, G. Bayona, M. Rueda, M. Paez-Reyes, D. Cárdenas, A. Duque, J.L. Crowley, Carlos Santos and Daniele Silvestro. 2021. Extinction at the end-Cretaceous and the origin of modern Neotropical Rainforests. Science, (372): 63-68.

Celi, J., E. Ternerus, J. Torres and M. Ortega. 2004. Dung beetles (Coleoptera: Scarabaeinae) diversity in an altitudinal gradient in the Cutucú Range, Morona Santiago, Ecuadorian Amazon. Lyonia, 7(2): 37-52.

Chamorro, W., D. Marín-Armijos, V. Granda and F.Z. Vazde-Mello. 2018. Listado de especies y clave de géneros y subgéneros de escarabajos estercoleros (Coleoptera: Scarabaeidae: Scarabaeinae) presentes y presuntos para Ecuador. Revista Colombiana de Entomología, 44(1): 72-100.

Chamorro, W., D. Marín-Armijos, A. Asenjo and F.Z. Vazde-Mello. 2019. Scarabaeinae dung beetles from Ecuador: a catalog, nomenclatural acts, and distribution records. ZooKeys, (826): 1-343.

Cokendolpher, J.C. and V.J. Polyak. 1996. Biology of the caves at Sinkhole Flat, Eddy county, New Mexico. Journal of Cave and Karst Studies, 58(3): 181-192.

Creedy, T.J. and D.J. Mann. 2011. Identification guide to the Scarabaeinae dung beetles of Cusuco National Park, Honduras. Version 1.0. Operation Wallacea, Lincolnshire.

Cultid-Medina, C.A., C.A. Medina-Uribe, B.G. Martínez-Quintero, A.F. Escobar-Villa, L.M. Constantino and N.J. Betancur-Posada. 2012. Escarabajos coprófagos (Scarabaeinae) del Eje Cafetero: guía para el estudio ecológico. Wildlife Conservation Society Colombia- CENICAFÉ-Federación Nacional de Cafeteros de Colombia, Villa María.

Delgado, J.M., A.E. Castro-Ramírez, M.A. Morón y L. Ruiz-Montoya. 2012a. Diversidad de Scarabaeoidea (Coleoptera) en las principales condiciones de hábitat de Montebello, Chiapas, México. Acta Zoológica Mexi- cana (n.s.), 28(1): 185-210.

Delgado, L. 1995. Onthophagus luismargaritorum, nueva especie mexicana del grupo clypeatus (Coleóptera: Scarabaeidae). Folia Entomológica Mexicana, (94): 57-61.

Delgado, L. 1997. Distribución estatal de la diversidad y nuevos registros de Scarabaeidae (Coleoptera) Mexicanos. Folia Entomológica Mexicana, (99): 37-56.

Delgado, L. 1999. Una nueva especie de Onthophagus asociada a Madrigueras de mamíferos, con nuevos registros para otros Scarabaeinae mexicanos (Coleóptera: Scarabaeidae). Dugesiana, 6(1): 33-39.

Delgado, L. and F. Capistan. 1996. A new Mexican species of Onthophagus (Coleoptera, Scarabaeidae) of the chevrolati group. Revista Brasileira Entomologica, 40(2): 157-158.

Delgado, L. and D. Curoe. 2014. Panamanian Onthophagus (Coleoptera: Scarabaeidae): description of a new species, and a revised key to the especies. Florida Entomologis, 97(1): 61-67.

Delgado, L. y C. Deloya. 1990. Una nueva especie de Onthophagus Latreille, 1802, del grupo clypeatus (Coleoptera: Scarabaeidae: Scarabaeinae). Annales de la Société Entomologique de France (N.S.), 26(2): 211-216.

Delgado, L. y H.F. Howden. 2000. Una nueva especie de Onthophagus de México (Coleoptera: Scarabaeidae: Scarabaeinae). Folia Entomológica Mexicana, (109): 35-41.

Delgado, L. y E. Montes de Oca. 2005. Los Escarabajos copro-necrófagos. (pp. 405-416). En: Sánchez-Ramos, G., P. Reyes-Castillo y R. Dirzo (Eds.). Historia Natural de la Reserva de la Biósfera El Cielo, Tamaulipas, México. Universidad Autónoma de Tamaulipas, Hong Kong.

Delgado, L. and E.F. Mora-Aguilar. 2019. A new Mexican species of Onthophagus Latreille (Coleoptera: Scarabaeidae: Scarabaeinae), with a revised key to the species of the $O$. dicranius species complex. Zootaxa, 4695(6): 586-592.

Delgado, L. y M. Pensado. 1998. Una nueva especie mexicana de Onthophagus del grupo clypeatus (Coleoptera: Scarabaeidae). Folia Entomologica Mexicana, (103): 75-80.

Delgado, L., J.L. Navarrete-Heredia and J. Blackaller. 1993. A new mexican species of Onthophagus with mycophagous habits (Coleoptera: Scarabaeidae: Scarabaeinae). The Coleopterists Bulletin, 47(2): 121-126.

Delgado, L., L.N. Peraza and C. Deloya. 2006. Onthophagus yucatanus, a new species of the clypeatus group from Mexico and Guatemala. Florida Entomologist, 89(1): 6-9.

Delgado, L., E.F. Mora-Aguilar and F. Escobar-Hernández. 2012. Scarabaeoidea (Coleoptera) of the municipality of Xalapa, Veracruz, Mexico: inventory and analysis. The Coleopterists Bulletin, 66(4): 319-332.

Dellacasa, M., R. Gordon and G. Dellacasa. 2004. Neotrichonotulus, a new genus for three Mexican Aphodiini (Scarabaoidea, Aphodiidae). Acta Zoológica Mexicana (n.s.), 20(2): 1-7.

Deloya, C. 1988. Coleópteros lamelicornios asociados a depósitos de detritos de Atta mexicana (Smith) en el 
sur del estado de Morelos, México. Folia Entomológica Mexicana, (75): 77-121.

Deloya, C. 1992a. Necrophilous Scarabaeidae and Trogidae beetles of tropical deciduous forest in Tepexco, Puebla, México. Acta Zoológica Mexicana (n.s.), (52): 1-11.

Deloya, C. 1992b. Lista de las especies de Coleoptera Lamellicornia del estado de Veracruz, México (Passalidae, Trogidae, Lucanidae, Scarabaeidae y Melolonthidae). Boletín de la Sociedad Veracruzana de Zoología, 2(2): 19-32.

Deloya, C. and M.A. Morón. 1998. Scarabaeoidea (Insecta: Coleoptera) necrófagos de "Los Tuxtlas", Veracruz y Puerto Ángel, Oaxaca, México. Dugesiana, 5(2): 17-28.

Deloya, C. y D. Covarrubias-Melgar. 2014 (Eds.). 2014. Escarabajos del estado de Guerrero (Coleoptera: Scarabaeoidea). S y G Editores, Ciudad de México.

Deloya, C., A. Burgos, J. Blackaller y J.M. Lobo. 1993. Los coleópteros lamelicornios de Cuernavaca, Morelos, México (Passalidae, Trogidae, Scarabaeidae y Melolonthidae). Boletín de la Sociedad Veracruzana de Zoología, 3(1): 15-55.

Deloya, A., V. Parra-Tabla y H. Delfín-González. 2007. Fauna de coleópteros Scarabaeidae Laparosticti y Trogidae (Coleoptera: Scarabaeoidea) asociados al bosque mesófilo de montaña, cafetales bajo sombra y comunidades derivadas en el centro de Veracruz, México. Neotropical Entomology, 36(1): 5-21.

Deloya, C., M. Madora-A. y D. Covarrubias-M. 2013. Scarabaeidae y Trogidae (Coleoptera) necrófilos de Acahuizotla, Guerrero, México. Revista Colombiana de Entomología, 39 (1): 88-94.

Deloya, C., J. Ponce-Saavedra, P. Reyes-Castillo y G. Aguirre-León (Eds.). 2016. Escarabajos del estado de Michoacán (Coleoptera: Scarabaeoidea). S y G Editores, Ciudad de México.

Deloya-López, A.C. 2011. Escarabajos coprófagos y necrófagos (Insecta: Coleoptera: Scarabaeidae). (pp. 383390). En: Cruz-Angón, A (Ed.). La biodiversidad en Veracruz, Estudio de Estado. Volumen II. Comisión Nacional para el Conocimiento y Uso de la Biodiversidad, Gobierno del Estado de Veracruz, Universidad Veracruzana, Instituto de Ecología, A.C., Ciudad de México, Xalapa.

Deloya-López, A.C. y E.A. López-Huicochea. 2012. Diversidad de escarabajos copro-necrófagos (Coleoptera: Scarabaeoidea), de la finca "El Mirador", Totutla, Veracruz, México. Entomología Mexicana, 12(1): 540-545.

Díaz, A., E. Galante and M.E. Favila. 2010. The effect of the landscape matrix on the distribution of dung and carrion beetles in a fragmented tropical rain forest. Journal of Insect Science, 10(81): 1-16.

Díaz, A., E. Galante y M.E. Favila. 2011. Escarabajos del estiércol en la selva fragmentada de Los Tuxtlas. (pp. 469-481). En: Cruz-Angón, A (Ed.). La biodiversidad en Veracruz, Estudio de Estado. Volumen II. Comisión Nacional para el Conocimiento y Uso de la Biodiversidad, Gobierno del Estado de Veracruz, Universidad Veracruzana, Instituto de Ecología, A.C., Ciudad de México y Xalapa.
Edmonds, W.D. 2004. A new species of Phanaeus MacLeay (Coleoptera: Scarabaeidae, Scarabaeinae) from Sonora, Mexico. The Coleopterists Bulletin, 58(1): 119-124.

Edmonds, W.D. 2018. The dung beetle fauna of the Big Bend region of Texas (Coleoptera: Scarabaeidae: Scarabaeinae). Insecta Mundi, (642): 1-30.

Edmonds, W.D. and G. Halffter. 1978. Taxonomic review of the subfamily Scarabaeidae. Systematics Entomology, (3): 307-331.

Emlen, D. 1997. Alternative reproductive tactics and male-dimorphism in the horned beetle Onthophagus acuminatus (Coleoptera: Scarabaeidae). Behavioral Ecology and Sociobiology, (41): 335-341.

Emlen, D. 2000. Integrating development with evolution: A case study with beetle horns. Bioscience, (50): 5.

Emlen, D. 2001. Costs and the diversification of exaggerated animal structures. Science, (291): 1534-1536.

Emlen, D. and H.F. Nijhout. 2000. The development and evolution of exaggerated morphologies in insects. Annual Review of Entomology, (45): 661-708.

Escobar, F. 1997. Estudio de la comunidad de Coleópteros Coprófagos (Scarabaeidae) en un remanente de bosque seco al Norte del Tolima, Colombia. Caldasia, 19(3): 419-430.

Escobar, F. 2000. Diversidad y distribución de los escarabajos del estiércol (Coleóptera: Scarabaeidae: Scarabaeinae) de Colombia. (pp. 197-210). En: Martín-Piera, F y J.J. Morrone (Eds.). Hacia un proyecto CYTED para el inventario y estimación de la diversidad entomológica en Iberoamérica: PrIBES-2000. Monografías 3ercer Milenio M3M, Sociedad Entomológica Aragonesa, Zaragoza.

Escobar, F. 2004. Diversity and composition of dung beetle (Scarabaeidae) assemblages in a heterogeneous Andean landscape. Tropical Zoology, (17): 123-136.

Escobar, F. y P. Chacón de Ulloa. 2000. Distribución espacial y temporal en un gradiente de sucesión de la fauna de coleópteros coprófagos (Scarabaeinae, Aphodiinae) en un bosque tropical montano, Nariño - Colombia. Revista de Biología Tropical, 48(4): 961-975.

Escobar, F. y C. Medina. 1996. Coleópteros coprófagos (Scarabaeidae) de Colombia: Estado actual de su conocimiento. (pp. 93-115.). En: Amat, G., G. Andrade y F. Fernández (Eds.). Insectos de Colombia, trabajos escogidos. Pontificia Universidad Javeriana, Santafé de Bogotá, D. C.

Escobar, F., G. Halffter and L. Arellano. 2007. From forest to pasture: an evaluation of the influence of environment and biogeography on the structure of dung beetle (Scarabaeinae) assemblages along three altitudinal gradients in the Neotropical region. Ecography, (30): 193-208.

Escobar, F., G. Halffter, Á. Solís, V. Halffter and D. Navarrete. 2008. Temporal shifts in dung beetle community structure within a protected area of tropical wet forest: a 35-year study and its implications for long-term conservation. Journal of Applied Ecology, (45): 1584-1592.

Escobar, F., P. Koleff y M. Rös, 2009. Evaluación de capacidades para el conocimiento: el Sistema Nacional 
de Información sobre Biodiversidad como un estudio de caso. (pp. 23-49). En: CONABIO y PNUD (Eds.). México, capacidades para la conservación y el uso sustentable de la biodiversidad. Comisión Nacional para el Conocimiento y Uso de la Biodiversidad-Programa de Naciones Unidas para el Desarrollo, Ciudad de México.

Escobar, F., J.M. Lobo, G. Halffter, F. Vaz-de-Mello, M. Dellacasa y F. Cabrero-Sañudo F. 2015. Distribución potencial en taxones hiperdiversos y poco conocidos (Insecta: Coleoptera: Scarabaeoidea): estimando la localización de nuevos taxones y el efecto de los cambios en los usos del suelo. Reporte técnico final. Comisión Nacional para el Conocimiento y Uso de la Biodiversidad, Ciudad de México.

Escobar-Hernández, F., A. Arenas-Parral and A. Arriaga-Jiménez. 2019. New distribution records and ecological notes for seven species of Onthophagus Latreille (Coleoptera: Scarabaeidae) in Oaxaca, Mexico, with description of the female of Onthophagus howdeni Zunino and Halffter and redescription of Onthophagus zapotecus Zunino and Halffter. The Coleopterists Bulletin, 73(3): 535-550.

Esparza-León, A.C. y G.D. Amat-García. 2007. Composición y riqueza de escarabajos coprófagos (Coleoptera: Scarabaeidae: Scarabaeinae) en un gradiente altitudinal de selva húmeda tropical del Parque Nacional Catatumbo-Barí (Norte de Santander), Colombia. Actualidades Biológicas, 29(87): 187-198.

Estrada, A. and R. Coates-Estrada. 2002. Dung beetles in continuous forest, forest fragments and in an agricultural mosaic habitat island at Los Tuxtlas, Mexico. Biodiversiy and Conservation, (11): 1903-1918.

Estrada, A., G. Halffter, R. Coates-Estrada and D.A. Meritt, Jr. 1993. Dung beetles attracted to mammalian herbivore (Alouatta palliata) and omnivore (Nasua narica) dung in the tropical rain forest of Los Tuxtlas, Mexico. Journal of Tropical Ecology, (9): 45-54.

Estrada, A., R. Coates-Estrada, A. Anzures-Dadda and P. Cammarano. 1998. Dung and carrion beetles in tropical rain forest fragments and agricultural habitats at Los Tuxtlas, Mexico. Journal of Tropical Ecology, (14): 577-593.

Favila, M.E. 2012. Historical, biogeographical and Ecological factors explain the success of some native dung beetles after the introduction of cattle in Mexico. Pastos, 42(2): 161-181.

Favila, M.E. 2017. Capítulo 22. Los escarabajos del estiércol en la Estación de Biología Tropical Los Tuxtlas. (pp. 461-472.). En: Reynoso, V.H., R.I. Coates y M.L. Vázquez-Cruz. Avances y perspectivas en la investigación de los bosques tropicales y sus alrededores: La región de Los Tuxtlas. Instituto de Biología, Universidad Nacional Autónoma de México, Ciudad de México.

García-Mendoza, A.J., M.J. Ordoñez y M. Briones-Salas (Eds.). 2004. Biodiversidad de Oaxaca. Instituto de Biología de la Universidad Autónoma de México, Fondo Oaxaqueño para la Conservación de la Naturaleza, World Wildlife Fund, Ciudad de México.

García-Morales, L.J y E. Montes de Oca. 2005. Diversidad de los escarabajos (Coleoptera: Scarabaeoidea: Scarabaeidae, Melolonthidae, Lucanidae, Passalidae y Trogidae) en el estado de Tamaulipas, México. (pp. 125-137). En: Barrientos-Lozano, L., A. Correa-Sandoval, J.V. Horta-Vega y J. García-Jiménez. Biodiversidad tamaulipeca. Vol. 1. Dirección General de Educación Superior Tecnológica, Instituto Tecnológico de Cd. Victoria, Ciudad Victoria.

Gasca-Álvarez, H.J., M. Zunino and C. Deloya. 2018. The ninth brachypterous Onthophagus Latreille (Coleoptera: Scarabaeidae: Scarabaeinae) of the world: a new species from Mexico. Journal of Natural History, 52(33-34): 2121-2132.

Génier, F. 2017. A new Guatemalan cloud forest endemic Onthophagus Latreille, 1802 (Coleoptera: Scarabaeidae: Scarabaeinae). The Canadian Entomologist, 149(5): 574-580.

Génier, F. and H.F. Howden. 1999. Two new central American Onthophagus Latreille of the mirabilis species group (Coleoptera: Scarabaeidae, Scarabaeinae). The Coleopterists Bulletin, 53(2): 130-144.

Gill, B.D. 1991. Dung beetles in tropical American forest. (pp. 211-229). En: Hanski, I. and Y. Cambefort (Eds.). Dung beetle ecology. Princeton University Press, Princeton.

Giraldo-Echeverri, C., S. Montoya-Molina y F. Escobar-Sarria. 2018. Escarabajos del estiércol en paisajes ganaderos de Colombia. Fundación CIPAV, Cali.

Goidanich, A e E. Malan. 1964. Sulla nidificazione pedotrofica di alcune specie di Onthophagus europei e sulla microflora aerobica dell'apparato digerente delle larve di Onthophagus taurus Schreber (Coleoptera Scarabaeidae). Annali della Facoltà di Scienze Agrarie della Università degli Studi di Torino, (2): 213-378.

González-Alvarado, A. y C.A. Medina. 2015. Listado de especies de escarabajos coprófagos (Coleoptera: Scarabaeidae: Scarabaeinae) de bosque seco de Colombia. Biota Colombiana, 16(1): 36-44.

González-Alvarado, A., E. Torres y C.A. Medina. 2015. Escarabajos coprófagos (Coleoptera: Scarabaeidae: Scarabaeinae) de bosques secos colombianos en la Colección Entomológica del Instituto Alexander von Humboldt. Biota Colombiana, 16(1): 88-95.

González-Hernández, A.L., J.L. Navarrete-Heredia, G.A. Quiroz-Rocha y C. Deloya. 2015. Coleópteros necrócolos (Scarabaeidae: Scarabaeinae, Silphidae y Trogidae) del bosque Los Colomos, Guadalajara, Jalisco, México. Revista Mexicana de Biodiversidad, (86): 764-770.

González-Maya, J.F. and J. Mata-Lorenzen. 2008. Dung-beetles (Coleoptera: Scarabaeidae) from the Zona Protectora Las Tablas, Talamanca, Costa Rica. Check List, 4(4): 458-463.

González-Tokman, D., I. Martínez, Y. Villalobos-Ávalos, R. Munguía-Steyer, M.R. Ortiz-Zayas, M- Cruz-Rosales and J.P. Lumaret. 2017. Ivermectin alters reproductive success, body condition and sexual trait expression in dung beetles. Chemosphere, (178): 129-135.

González-Tokman, D., C. Cultid-Medina, A. Díaz, F. Escobar, L Ocampo-Palacio and C. Martínez-Garza. 2018. 
Success or failure: the role of ecological restoration on the recovery of dung beetle diversity and function in a tropical rainforest. Revista Mexicana de Biodiversidad, (89): 232-242.

Granados, J.M, B. Kohlmann y R. Russo. 2010. Escarabajos del estiércol como bioindicadores del impacto ambiental causado por cultivos en la región atlántica de Costa Rica. Tierra Tropical, 6(2): 181-189.

Guérin-Méneville, F.E. 1855. Catalogue des Insectes Coléoptères, recueillis par M. Gaetano Osculati, pendant son exploration de la región équatoriale, sur les bords du Napo et de l'Amazone. Verhandlungen des Zoologisch-Botanischen Vereins in Wien, (5): 573-612.

Gunter, N.L., T.A. Weir, A. Slipinksi, L. Bocak and S.L. Cameron. 2016. If dung beetles (Scarabaeidae: Scarabaeinae) arose in association with dinosaurs, did they also suffer a mass co-extinction at the K-Pg boundary? PLoS ONE, 11(5): e0153570.

Halffter, G. 1962. Explicación preliminar de la distribución geográfica de los Scarabaeinae mexicanos. Acta Zoológica Mexicana (n.s.), (5): 1-17.

Halffter, G. 1964. La entomofauna americana, ideas acerca de su origen y distribución. Folia Entomológica Mexicana, (6): 1-108.

Halffter, G. 2017. La zona de transición mexicana y la megadiversidad de México: del marco histórico a la riqueza actual. Dugesiana, 24(2): 77-89.

Halffter, G. 2019. Capítulo 6. La Zona de Transición Mexicana: Referente obligado para una nueva ponderación de la riqueza biológica de México. (pp. 129-155). En: Moreno, C.E. (Ed.). La biodiversidad en un mundo cambiante: Fundamentos teóricos y metodológicos para su estudio. Universidad Autónoma del Estado de Hidalgo, Libermex, Ciudad de México.

Halffter, G. and L. Arellano. 2002. Response of dung beetle diversity to human-induced changes in a tropical landscape. Biotropica, 34(1): 144-154.

Halffter, G. y C. Deloya. 2007. Primer caso de nidificación de un Geotrupino (Scarabaeoidea: Geotrupidae) en cuevas: un fenómeno excepcional. Acta Zoológica Mexicana (n.s.), 23(3): 139-142.

Halffter, G. and V. Halffter. 2009. Why and where coprophagous beetles (Coleoptera: Scarabaeinae) eat sedes, fruits or vegetable detritus. Boletín Sociedad Entomológica Aragonesa, (45): 1-22.

Halffter, G. y E.G. Matthews. 1966. The natural history of dung beetles of the subfamily Scarabaeinae. Folia Entomológica Mexicana, (12-14): 1-312.

Halffter, G. and J.J. Morrone. 2017. An analytical review of Halffter's Mexican transition zone, and its relevance for evolutionary biogeography, ecology and biogeographical regionalisation. Zootaxa, 4226(1): 1-46.

Halffter, G., M.E. Favila and V. Halffter. 1992. A comparative study of the structure of the scarab guild in Mexican tropical rain forests and derived ecosystems. Folia Entomológica Mexicana, (84): 131-156.

Halffter, G., M.E. Favila and L. Arellano. 1995. Spatial distribution of three groups of Coleoptera along an altitudinal transect in the Mexican Transition Zone and its biogeographical implications. Elytron, (9): 151-185. Halffter, G., E. Pineda, L. Arellano and F. Escobar. 2007. Instability of copronecrophagous beetle assemblages (Coleoptera: Scarabaeinae) in a mountainous tropical landscape of Mexico. Environmental Entomology, 36(6): 1397-1407.

Halffter, G., J.R. Verdú, J. Márquez and C.E. Moreno. 2008. Biogeographical analysis of Scarabaeinae and Geotrupinae along a transect in central Mexico. Fragmenta Entomológica, 40(2): 273-322.

Halffter, G., J.R. Verdú, C.E. Moreno and V. Halffter. 2012. Historical and ecological determinants of dung beetle assemblages in two arid zones of central Mexico. Journal of Arid Environments, (76): 54-60.

Halffter, G., M. Zunino, V. Moctezuma and J.L. Sánchez-Huerta. 2019. The integration processes of the distributional patterns in the Mexican Transition Zone: Phyletic, paleogeographic and ecological factors of a case study. Zootaxa, 4586(1): 1-34.

Hanski, I. and Y. Cambefort. 1991. Spatial processes. (pp. 283-304). En: Hanski, I. and Y. Cambefort (Eds.). Dung beetle ecology. Princeton University Press, Princeton.

Harold, E. 1869. Note sur quelques coprides du Mexique. Annales de la Société Entomologique de France, (4): 439-512.

Harold, E. 1871. Diagnosen neuer Coprophagen. Coleopterologische Hefte, (8): 114-116.

Harold, E. 1873. Diagnosen neuer Coprophagen. Coleopterologische Hefte, (11): 102-105.

Harold, E. 1880. Verzeichniss der von E. Steinheil in Neu-Granada gesammelten Coprohagen Lamellicornien. Stettiner Entomologische Zeitung, (41): 13-46.

Henshaw, S. 1885. List of the Coleoptera of America, North of Mexico. American Entomological Society, Filadelfia.

Hernández, B. and J.L. Navarrete-Heredia. Annotated checklist and biogeographical affinities of Scarabaeinae beetles from Los Altos de Jalisco Region, Mexico. Southwestern Entomologist, 43(1): 131-150.

Hernández, B., J. Maes, C. Harvey, S. Vilchez, A. Medina y D. Sánchez. 2003. Abundancia y diversidad de escarabajos coprófagos y mariposas diurnas en un paisaje ganadero en el departamento de Rivas, Nicaragua. Agroforestería en las Américas, (10): 93-102.

Hernández, B., J.L. Barragán-Ramírez, J.L. Navarrete-Heredia, G.A. Quiroz-Rocha and M. Vásquez-Bolaños. 2021. Dung-beetle (Coleoptera: Scarabaeidae: Scarabaeinae) assemblage in two livestock production systems in a southern Mexican High Plateau semiarid ecosystem. The Canadian Entomologist, 153(3): 285300.

Hernández-Córdoba, H., A. P. Martínez-Falcón y C.E. Moreno. 2015. Sobrelapamiento de los patrones de actividad y redes ecológicas de escarabajos coprófagos (Scarabaeinae), en un potrero de la Huasteca Hidalguense. Entomología Mexicana, (3): 481-487.

Hoffmann, A., M.G. López-Campos y I.M. Vázquez-Rojas. 2004. Los artrópodos de las cavernas de México. (pp. 229-326). En: Llorente-Bousquets, J.J., J. Morrone, O. Yáñez-Ordóñez y I. Vargas-Fernández (Eds.). Biodiver- 
sidad, taxonomía y biogeografía de artrópodos de México: hacia una síntesis de su conocimiento. Universidad Nacional Autónoma de México, Ciudad de México.

Horgan, F.G. 2007. Dung beetles in pasture landscapes of Central America: proliferation of synanthropogenic species and decline of forest specialists. Biodiversity and Conservation, (16): 2149-2165.

Horgan, F.G. 2008. Dung beetle assemblages in forests and pastures of El Salvador: a functional comparison. Biodiversity and Conservation, (17): 2961-2978.

Horn, G.H. 1881. Descriptions of new species of North American Coleoptera. Transactions of the American Entomological Society, (7): 74-77.

Howden, H.F. 1973. Four new species of Onthophagus from Mexico and the United States (Coleoptera, Scarabaeidae). Proceedings of the Entomological Society of Washington, 75(3): 329-337.

Howden, H.F. and O.L. Cartwright. 1963. Scarab beetles of genus Onthophagus Latreille North of Mexico (Coleoptera: Scarabaeidae). Proceedings of the United States National Museum, 114(3467): 1-133.

Howden, H.F. and O.L. Cartwright. 1970. A new name for Onthophagus monticolus Howden \& Cartwright (Coleoptera: Scarabaeidae). Proceedings of the Entomological Society of Washington, 72(1): 54.

Howden, H.F. and F. Génier. 2004. Seven new species of Onthophagus Latreille from Mexico and the United States (Coleoptera: Scarabaeidae, Scarabaeinae). Fabreries, 28(1): 53-76.

Howden, H.F. and B.D. Gill. 1987. New species and new records of Panamanian and Costa Rican Scarabaeinae (Coleoptera, Scarabaeidae). The Coleopterists Bulletin, 41(3): 201-224.

Howden, H.F. and B.D. Gill. 1993. Mesoamerican Onthophagus Latreille in the dicranius and mirabilis species groups (Coleoptera, Scarabaeidae). The Canadian Entomologist, (125): 1091-1114.

Howden, H.F. and C.H. Scholtz. 1986. Changes in Texas dung beetle community between 1975 and 1985 (Coleoptera: Scarabaeidae, Scarabaeinae). The Coleopterists Bulletin, 40(4): 313-316.

Howden, H.F and O.P. Young. 1981. Panamanian Scarabaeinae: taxonomy, distribution and habits. Contributions of the American Entomological Institute, 18(1): 1-204.

Howden, H.F., O.L. Cartwright y G. Halffter. 1956. Descripción de una nueva especie mexicana de Onthophagus con anotaciones ecológicas sobre especies asociadas a nidos de animales y a cuevas. Acta Zoológica Mexicana, 1(9): 1-16.

Huerta, C. and M. García-Hernández. 2013. Nesting behavior of Onthophagus incensus Say, 1835 (Coleoptera: Scarabaeidae: Scarabaeinae). The Coleopterists Bulletin, 67(2): 161-166.

Huerta, C., I. Martínez and M. García-Hernández. 2010. Preimaginal development of Onthophagus incensus Say, 1835 (Coleoptera: Scarabaeidae: Scarabaeinae). The Coleopterists Bulletin, 64(4): 365-371.

Huerta, C., L. Arellano, M. Cruz, F. Escobar e I. Martínez.
2016. Los escarabajos del estiércol en los potreros ganaderos de Xico. Instituto de Ecología, A.C., Xalapa.

Huerta, C., L. Arellano and M. Cruz. 2019. Dung beetles (Coleoptera: Scarabaeidae, Scarabaeinae) and dung removal in Mexican livestock pastures. Revista Mexicana de Biodiversidad, (89): 1280-1292.

Huerta-Crespo, C. y M. Cruz-Rosales. Hacia una ganadería sustentable y amigable con la biodiversidad. Estudio de caso: Xico, Veracruz. Instituto de Ecología, A.C., Xalapa.

Hunt, J. and L. Simmons. 1997. Patterns of fluctuating asymmetry in beetle horns: an experimental examination of the honest signaling hypothesis. Behavioral Ecology and Sociobiology, (41): 109-114.

CINZ. 1999. Código Internacional de Nomenclatura Zoológica. $4^{a}$ edición. Comisión Internacional de Nomenclatura Zoológica, Londres.

Inward, D.J.G., R.G. Davies, C. Pergande, A.J. Denham and A.P. Vogler. 2011. Local and regional ecological morphology of dung beetle assemblages across four biogeographic regions. Journal of Biogeography, (38): 1668-1682.

Jaramillo, C., 2019. 140 million years of tropical biome evolution. (pp. 1-28). En: Gómez, J. and A.O. Pinilla-Pachon (Eds.). The Geology of Colombia, Volume 2. Mesozoic. Servicio Geológico Colombiano, Publicaciones Geológicas Especiales 36, Bogotá.

Jiménez-Sánchez, E., G. Labrador-Chávez, E. López-Contreras, J.L. Navarrete-Heredia y J. Padilla-Hernández. 2009. Apéndice II. Especies de las familias Staphylinidae, Silphidae, Scarabaeidae y Trogidae del Estado de México. (pp. 395-397). En: Ceballos, G., R. List, G. Garduño, R. López-Cano, M.J. Muñozcano Quintanar, E. Collado y J.E. San Román. La diversidad biológica del Estado de México. Estudio de Estado. Gobierno del Estado de México, Toluca de Lerdo.

Joaqui, T., V. Moctezuma, J.L. Sánchez-Huerta and F. Escobar. 2019. The Onthophagus fuscus (Coleoptera: Scarabaeidae) species complex: an update and the description of a new species. Zootaxa, 4555(2): 151-186.

Joaqui, T., C.A. Cultid-Medina, W. Dáttilo and F. Escobar. 2021. Different dung beetle diversity patterns emerge from overlapping biotas in a large mountain range of the Mexican Transition Zone. Journal of Biogeography.

Knell, R. 2011. 3. Male contest competition and the evolution of Weapons. (pp. 47-65). En: Simmons, L.W. and T.J. Ridsdill-Smith (Eds.). Ecology and evolution of dung beetles. Blackwell Publishng Ltd, Chichester.

Kohlmann, B. and S. Sánchez. 1984. Structure of a Scarabaeinae community: a numerical-behavioral study (Coleoptera: Scarabaeidae). Acta Zoológica Mexicana, 2: $1-27$.

Kohlmann, B. y Á. Solís. 2001. El género Onthophagus (Coleoptera: Scarabaeidae) en Costa Rica. Giornale Italiano di Entomologia, 49: 159-261.

Kohlmann, B., Á. Solís, E., Ortwin, X. Soto and R. Russo. 2007. Biodiversity, conservation, and hotspot atlas of Costa Rica: a dung beetle perspective (Coleoptera: Scarabaeidae: Scarabaeinae). Zootaxa, (1457): 1-34. 
Kohlmann, B., A. Arriaga-Jiménez and M. Rös. 2018. An unusual new species of Canthidium (Coleoptera: Scarabaeidae: Scarabaeinae) from Oaxaca, Mexico. Zootaxa 4378(2): 273-278.

Kohlmann, B., Á. Solís and G.E. Alvarado. 2019. Description of Onthophagus humboldti and Uroxys bonplan$d i$, two new scarab beetles (Coleoptera, Scarabaeidae, Scarabaeinae) from Costa Rica, with notes on tropical mountain brachyptery and endemicity. ZooKeys, (881): 23-51.

Kohlmann, B., A. Arriaga-Jiménez and M. Rös. 2020. Case 3807 - Onthophagus anthracinus Harold, 1873 (Insecta, Coleoptera, Scarabaeidae): proposed conservation of the specific name. Bulletin of Zoological Nomenclature, (77): 79-82.

Krajcik, M. 2006. Checklist of Scarabaeoidea of the World. 1. Scarabaeinae (Coleoptera: Scarabaeidae). Animma, 10(3): 1-190.

Lacordaire, T. 1856. Histoire naturelle des insectes. Genera des Coléoptères. Ou exposé méthodique et critique de tous les genres proposés jusqu'ici dans cet ordre d'insectes. Tome Troisième. Contenant les familles des Pectinicornes et Lamellicornes. Librairie Encyclopédique de Roret, París.

Larsen, T.H., A. Lopera and A. Forsyth. 2008. Understanding trait-dependent community disassembly: dung beetles, density functions, and forest fragmentations. Conservation Biology, 22(5): 1288-1298.

Latha, T., P.Y. Huang, G.A. Perez and I.O. Paquiul. 2016. Dung beetle assemblage in a protected area of Belize: A study on the consequence of forest fragmentation and isolation. Journal of Entomology and Zoology Studies, 4(1): 457-463.

Latreille, P.A. 1802. Histoire naturelle des crustacés et insectes. Imprimerie de F. Dufart, París.

Lavin, M. and M. Luckow. 1993. Origins and relationships of tropical North America in the context of the boreotropics hypothesis. American Journal of Botany, (80): $1-14$.

Leng, C.W. 1920. Catalogue of the Coleoptera of America, north of Mexico. John D. Sherman, Jr., Mount Vernon.

Leng, C.W. and A.J. Mutchler. 1933. Second and third supplements 1925 to 1932 (inclusive) to the catalogue of the Coleoptera of America, north of Mexico. John D. Sherman, Jr., Mount Vernon.

Lizardo, V. and I. Castellanos-Vargas. 2016. Dung beetle community response to vegetation type and season in an arid zone of the Mexican Plateau. Southwestern Entomologist, 41(2): 441-452.

Lizardo, V., F. Escobar and O. Rojas-Soto. 2017. Diversity and distribution of Phanaeini (Coleoptera: Scarabaeidae: Scarabaeinae) in Mexico. Zootaxa, 4358 (2): 271294.

Llorente-Bousquets, J. y S. Ocegueda. 2008. (pp. 283-322). Estado del conocimiento de la biota. En: CONABIO (Ed.). Capital natural de México. Volumen I. Conocimiento actual de la biodiversidad. Comisión Nacional para el Conocimiento y Uso de la Biodiversidad, Ciudad de México.
Lobo, J.M. y G. Halffter. 1994. Relaciones entre escarabajos (Coleoptera: Scarabaeidae) y nidos de tuza (Rodentia: Geomydae): implicaciones biológicas y biogeográficas. Acta Zoológica Mexicana (n.s.), (62): 1-9.

Lobo, J. and G. Halffter. 2000. Biogeografical and ecological factors affecting the altitudinal variation of mountainous communities of coprophagous beetles (Coleoptera: Scarabaeoidea): a comparative study. Annals of the Entomological Society of America, 93(1): 115-126.

Lumaret, J.P., N. Kadiri and I. Martínez-M. 2020. The global decline of dung beetles. (pp. 1-10). En: Reference Module in Earth Systems and Environmental Sciences. Elsevier, Amsterdam.

Maes, J.M. 1998. Insectos de Nicaragua. Volumen II. Imprenta PRINT-León, Managua.

Márquez, J., M.A. Morón, C. Moreno, J. Asiain y G. Sánchez-Rojas. 2017. Escarabajos de la familia Scarabaeidae (Insecta: Coleoptera). (pp. 389-412). En: Ramírez-Bautista, A., A. Sánchez-González, G. Sánchez-Rojas y C. Cuevas-Cardona. Biodiversidad del estado de Hidalgo. Tomo II. Universidad Autónoma del Estado de Hidalgo, Pachuca de Soto.

Martín-Piera, F. and J.M. Lobo. 1993. Altitudinal distribution patterns of copro-necrophagous Scarabaeidae (Coleoptera) in Veracruz, Mexico. The Coleopterists Bulletin, 47(4): 321-334.

Martínez, A. 1947. Addenda y corrigenda al trabajo de Blackwelder checklist of the coleopterous insects of Mexico, Central America, the West Indies, and South America (Scarabaeidae, Coprinae). Arthropoda, 1(1): 109-114.

Martínez, I. and M. Cruz. 1999. Comparative morphological analysis of testis follicles in dung beetles (Coleoptera: Scarabaeidae: Scarabaeinae, Aphodiinae, Geotrupinae). Proceedings of the Entomological Society of Washington, 101(4): 804-815.

Martínez, I. y J.P. Lumaret. 2003. Dimorfismo sexual en larvas de Scarabaeoidea (Coleoptera). (pp. 11-18). En: Onore, G., P. Reyes-Castillo y M. Zunino (Eds.). Escarabeidos de Latinoamérica: estado del conocimiento. Monografías 3ercer Milenio M3M, Sociedad Entomológica Aragonesa, Zaragoza.

Martínez, I., E. Montes de Oca y M. Cruz. 1998. Contribución al conocimiento del escarabajo coprófago $\mathrm{On}$ thophagus incensus Say (Coleoptera: Scarabaeidae: Scarabaeinae): datos ecológicos y reproductivos en relación a su fenología. Folia Entomológica Mexicana, (103): 1-13.

Martínez, I., M. Cruz, E. Montes de Oca y T. Suárez. 2011. La función de los escarabajos del estiércol en los pastizales ganaderos. Gobierno del Estado de Veracruz de Ignacio de la Llave, Instituto de Ecología, A.C., Xalapa.

Martínez, E., P. González y E. Morelli. 1999. Anormalidades en el aparato reproductor de una hembra de $O n$ thophagus hirculus Mannh. (Coleoptera, Scarabaeidae, Scarabaeinae). Acta Zoológica Mexicana, (76): 127129.

Martínez, I., A. Ramírez-Hernández y J.P. Lumaret. 2017. Medicinas veterinarias, plaguicidas, y los escarabajos 
del estiércol en la zona tropical de Palma Sola, Veracruz, México. Southwestern Entomologist, 42(2): 563574.

Martínez, N.J., H. García, L.A. Pulido, D. Ospino y J.C. Narváez. 2009. Escarabajos Coprófagos (Coleoptera: Scarabaeinae) de la Vertiente Noroccidental, Sierra Nevada de Santa Marta, Colombia. Neotropical Entomology, 38(6): 708-715.

Martínez-H, N.J., L.M. Cañas-M, J.L. Rangel-A, O. Blanco-R, J.D. Mendoza-P y S. Cohen-B. 2010. Coleópteros coprófagos (Scarabaeidae: Scarabaeinae) en la Reserva Natural Las Delicias (RND), Sierra Nevada de Santa Marta (SNSM), Colombia. Boletín Cientifico Centro de Museos, Museo de Historia Natural, 14(2): 187-200.

Martínez-Revelo, D.E., C. Castro-Moreno y C.E. Medina. 2018. Escarabajos coprófagos de la cuenca alta y media del río Bita, Vichada, Colombia. Biota Colombiana, 19(1): 226-235.

Mastretta-Yanes, A., A. Moreno-Letelier, D. Piñero, T.H. Jorgensen and B.C. Emerson. 2015. Biodiversity in the Mexican highlands and the interaction of geology, geography and climate within the Trans-Mexican Volcanic Belt. Journal of Biogeography, (42): 1586-1600.

Matthews, E.G. 1966. A taxonomic zoogeographic survey of the Scarabaeinae of the Antilles (Coleoptera: Scarabaeidae). Memoirs of the American Entomological Society, (21): 1-133.

Medina, C.A. y L.A. Pulido. 2009. Escarabajos coprófagos (Coleoptera: Scarabaeinae) de la Orinoquia colombiana. Biota Colombiana, 10 (1-2): 55-62.

Medina, C.A. y F.A. González. 2014. Escarabajos coprófagos de la subfamilia Scarabaeinae. (pp. 195-213. En: Pizano, C. y H. García (Ed.). El bosque seco tropical en Colombia. Instituto de Investigación de Recursos Biológicos Alexander von Humboldt, Bogotá, D.C.

Medina, C., A. Lopera-Toro, A. Vítolo y B.D. Gill. 2001. Escarabajos Coprófagos (Coleóptera: Scarabaeidae: Scarabaeinae) de Colombia. Biota Colombiana, 2(2): 131-144.

Miranda-Flores, K.P., I.A. Chamorro-Florescano, M.E. Favila, J.L. Alanís-Méndez y M. Ortiz-Domínguez. 2020. Diversidad del paisaje y remoción del estiércol por escarabajos coprófagos en pastizales del norte de Veracruz. Revista Mexicana de Biodiversidad, (91): e912792.

Moctezuma, V. 2021. Spatial autocorrelation in a Mexican dung beetle ensemble: Implications for biodiversity assessment and monitoring. Ecological Indicators, (125): 107548.

Moctezuma, V. and G. Halffter. 2019a. New biogeographical makeup for colonisation of the Baja California Peninsula, with the description of a new Onthophagus (Coleoptera: Scarabaeidae: Scarabaeinae). Journal of Natural History, 53(33-34): 2057-2071.

Moctezuma, V. and G. Halffter. 2019b. Una especie nueva de México y un nuevo registro para Guatemala del complejo de especies Onthophagus dicranius (Coleoptera: Scarabaeidae). Revista Mexicana de Biodiversidad, (90): e903015.

Moctezuma, V. and G. Halffter. 2020a. Three new species of the Onthophagus chevrolati species group (Coleoptera: Scarabaeoidea: Scarabaeinae). Biologia, (75): $2277-$ 2286.

Moctezuma, V. and G. Halffter. 2020b. New species and redescriptions of the Onthophagus chevrolati species complex (Coleoptera: Scarabaeoidea: Scarabaeinae). Annales Zoologici, 70(2): 245-261.

Moctezuma, V. and G. Halffter. 2020c. A new species of the Onthophagus cyanellus species complex (Coleoptera: Scarabaeidae: Scarabaeinae: Onthophagini). The Coleopterists Bulletin, 74(3): 495-501.

Moctezuma, V. and G. Halffter. 2021. Species redescriptions and new species of the Onthophagus mexicanus species group (Coleoptera: Scarabaeidae), with notes on distribution and rodent-dung beetle associations. Zoological Studies, (60): 0dd.

Moctezuma, V., M. Rossini, M. Zunino and G. Halffter. 2016a. A contribution to the knowledge of the mountain entomofauna of Mexico with a description of two new species of Onthophagus Latreille, 1802 (Coleoptera, Scarabaeidae, Scarabaeinae). ZooKeys, 572: 23-50.

Moctezuma, V., G. Halffter and F. Escobar. 2016b. Response of copronecrophagous beetle communities to habitat disturbance in two mountains of the Mexican Transition Zone: influence of historical and ecological factors. Journal of Insect Conservation, (20): 945-956.

Moctezuma V., Gonzalo-Halffter and A. Arriaga-Jiménez. 2018. Archipelago reserves, a new option to protect montane entomofauna and beta-diverse ecosystems. Revista Mexicana de Biodiversidad, (89): 927-937.

Moctezuma, V., A. Espinosa de los Monteros y G. Halffter. 2019. Capítulo III. Filogenia Molecular de Scarabaeinae de Los Chimalapas: la selección de hábitat ligada a la historia evolutiva. (pp. 53-89). En: Moctezuma, V. Escarabajos del estiércol (Scarabaeinae) de Los Chimalapas, México: Análisis Taxonómico, Ecológico y Evolutivo. Tesis doctoral. Instituto de Ecología, A. C., Xalapa.

Moctezuma, V., J.L. Sánchez-Huerta and G. Halffter. 2020. Two new species of the Onthophagus clypeatus species group (Coleoptera: Scarabaeidae: Scarabaeinae). Florida Entomologist, 103(2): 281-287.

Moczek, A.P. and D.J. Emlen. 2000. Proximate determination of male horn dimorphism in the beetle Onthophagus taurus (Coleoptera: Scarabaeidae). Journal of Evolutionary Biology, (12): 27-37.

Monaghan, M.T., D.J.G. Inward, T. Hunt and A.P. Vogler. 2007. A molecular phylogenetic analysis of the Scarabaeinae (dung beetles). Molecular Phylogenetics and Evolution, (45): 674-692.

Montes de Oca, E. 2001. Escarabajos coprófagos de un escenario ganadero típico de la región de Los Tuxtlas, Veracruz, México: Importancia del paisaje en la composición de un gremio funcional. Acta Zoológica Mexicana (n.s.), (82): 111-132.

Montes de Oca, E. and G. Halffter. 1995. Daily and seasonal activities of a guild of the coprophagous, burrowing beetle (Coleoptera Scarabaeidae Scarabaeinae) in tropical grassland. Tropical Zoology, (8): 159-180. 
Montes-Rodríguez, J.M., O.F. Ortega-Molina y Y. Espinosa-Vélez. 2019. Efecto de borde en ensamblajes de escarabajos coprófagos (Coleoptera: Scarabaeidae) en fragmentos de bosque de un paisaje andino. Caldasia, 41(2): 380-391.

Montoya-Molina, S., C. Giraldo-Echeverri, J. Montoya-Lerma, J. Chará, F. Escobar and Z. Calle. 2015. Land sharing vs. land sparing in the dry Caribbean lowlands: A dung beetles' perspective. Applied Soil Ecology, (98): 204-212.

Montoya-Molina, S., L.M. Isaza-López y J. León-González. 2016. Escarabajos coprófagos (Scarabaeidae: Scarabaeinae) de la Cuenca del Río Dagua, Valle del Cauca, Colombia. Dugesiana, 23(2): 125-133.

Mora-Aguilar, E.F. y E. Montes de Oca. 2009. Escarabajos necrófagos (Coleoptera: Scarabaeidae y Trogidae) de la región central baja de Veracruz, México. Acta Zoológica Mexicana (n.s.), 25(3): 569-588.

Morales-Morales, C.J., F. Guevara-Hernández, J.A. Espinosa-Moreno, J.C. Gómez-Castañeda, J. Llaven-Martínez y R.C. Gutiérrez-Hernández. 2014. Escarabajos estercoleros (Coleoptera: Scarabaeidae) del Cutt San Ramón, Villaflores, Chiapas, México. Entomología Mexicana, (1): 1129-1132.

Moretto, P. 2010. Onthophagus, fruits and diplopods relationship in Senegal. Catharsius La Revue, (2): 11-18.

Moretto, P. et F. Génier. 2020. Nouveaux Onthophagus (sensu lato) Latreille, 1802, d'Afrique tropicale appartenant au groupe 23 de d'Orbigny (1913) (Coleoptera, Scarabaeidae, Onthophagini). Catharsius, (21): 4-23.

Morón, M.A. 1979. Fauna de coleópteros lamelicornios de la estación de biología tropical 'Los Tuxtlas', Veracruz, UNAM, México. Anales del Instituto de Biología de la Universidad Nacional Autónoma de México (Serie Zoología), (50): 375-454.

Morón, M.A. 1985. Fauna de coleópteros lamelicornios de Boca del Chajul, Chiapas, México. Folia Entomológica Mexicana, (66): 109-128.

Morón, M.A. 1987. The necrophagous Scarabaeinae beetles (Coleoptera, Scarabaeidae) from a coffee plantation in Chiapas, Mexico: Habits and phenology. The Coleopterists Bulletin, 41(3): 225-232.

Morón, M.A. 1993. Catálogo de tipos depositados en la colección M. A. Morón, Xalapa, Veracruz, México (MXAL) (Insecta, Coleoptera: Lamellicornia). Sociedad Mexicana de Entomología, A. C. y Sociedad Veracruzana de Zoología, A. C., Xalapa.

Morón, M.A. 2004. Escarabajos. 200 millones de años de evolución. Segunda edición. Instituto de Ecología A. C. y Sociedad Entomológica Aragonesa, Zaragoza.

Morón, M.A. y J. Blackaller. 1997. Melolonthidae y Scarabaeidae. (pp. 227-243). En: González-Soriano, E., R. Dirzo y R. Vogt (Eds.). Historia natural de Los Tuxtlas. Universidad Nacional Autónoma de México, Ciudad de México.

Morón, M.A. y C. Deloya. 1991. Los coleópteros lamelicornios de la Reserva de la Biósfera 'La Michilia', Durango, México. Folia Entomológica Mexicana, (81): 209-283.
Morón, M.A. and J. Márquez. 2012. New state and country records of scarab beetles (Coleoptera: Scarabaeoidea) and comments on their distribution. Revista Mexicana de Biodiversidad, (83): 698-711.

Morón, M.A. and R. Arce-Pérez. 2016. Soil scarab beetles at Santa Marta, Los Tuxtlas, Veracruz, Mexico. Southwestern Entomologist, 41(1): 51-62.

Morón, M.A., F.J. Villalobos y C. Deloya. 1985. Fauna de coleópteros lamelicornios de Boca de Chajul, Chiapas, México. Folia Entomológica Mexicana, (66): 57-118.

Morón, M.A., J.F. Canal y O. Canul. 1986. Análisis de la entomofauna necrófila del área norte de la Reserva de la Biosfera "Sian Ka'an", Quintana Roo, México. Folia Entomológica Mexicana, (69): 83-88.

Morón, M.A., A. Aragón, A. Tapia-Rojas y R. Rojas-García. 2000. Coleoptera Lamellicornia de La Sierra del Tentzo, Puebla, México. Acta Zoológica Mexicana, (79): 77-102.

Morón, M.A., A. Aragón-García y H. Carrillo-Ruiz (Eds.). 2013. Fauna de escarabajos del estado de Puebla. Miguel Ángel Morón Ríos, Coatepec.

Morrone, J.J. 2019. Regionalización biogeográfica y evolución biótica de México: encrucijada de la biodiversidad del Nuevo Mundo. Revista Mexicana de Biodiversidad, (90): e902980.

Morrone, J.J. 2020. The Mexican Transition Zone. A natural biogeographic laboratory to study biotic assembly. Springer Nature Switzeland AG, Cham.

Muñoz-Hernández, A., M.A. Morón y A. Aragón. 2008. Coleoptera Scarabaeoidea de la región de Teziutlán, Puebla, México. Acta Zoológica Mexicana (n.s.), 24(3): 55-78.

Naranjo-González, M. y L.J. Víctor-Rosas. 2015. La subfamilia Scarabaeinae (Coleoptera: Scarabaeidae) en la Colección Entomológica de la E.N.C.B., I.P.N. Entomología Mexicana, (2): 870-874.

Navarrete, D. and G. Halffter. 2008. Dung beetle (Coleoptera: Scarabaeidae: Scarabaeinae) diversity in continuous forest, forest fragments and cattle pastures in a landscape of Chiapas, Mexico: the effects of anthropogenic changes. Biodiversity and Conservation, (17): 2869-2898.

Navarrete-Gutiérrez, A. y G. Halffter. 2008. Nuevos registros de escarabajos copro-necrófagos (Coleoptera: Scarabaeidae: Scarabaeinae) para México y Chiapas. Acta Zoológica Mexicana (n.s.), 24(1): 247-250.

Navarrete-Heredia, J.L. y N.E. Galindo-Miranda. 1997. Escarabajos asociados a Basidiomycetes en San Jost de los Laureles, Morelos, México (Coleoptera: Scarabaeidae). Folia Entomológica Mexicana, (99): 1-16.

Navarrete-Heredia, J.L., L. Delgado y H.E. Fierros-López. 2001. Coleoptera Scarabaeoidea de Jalisco, México. Dugesiana, 8(1): 37-93.

Neita, J., L. Pardo, M. Quinto y N. Cuesta. 2003. Escarabajos copronecrófagos (Coleoptera: Scarabaeidae) en la parcela permanente de investigación en biodiversidad. (pp. 79-90). En: García-Cossio, F. (Ed.). Salero: Diversidad biológica de un bosque pluvial tropical (bp-T). Universidad Tecnológica del Chocó, Instituto de Inves- 
tigaciones Ambientales del Pacífico, Comunidad de Salero, Quibdó.

Nichols, E., T. Larsen, S. Spectro, A.L. Davis, F. Escobar, M. Favila, K. Vulinec and The Scarabaeinae Research Network. 2007. Global dung beetle response to tropical forest modification and fragmentation: A quantitative literatura review and meta-analysis. Biological Conservation, (137): 1-19.

Nichols, E., S. Spector, J. Louzada, T. Larsen, S. Amezquita, M.E. Favila and The Scarabaeinae Research Network. 2008. Ecological functions and ecosystem services provided by Scarabaeinae dung beetles. Biological Conservation, (141): 1461-1474.

Nijhout, H.F. and D.J. Emlen. 1998. Competition among body parts in the development and evolution of insect morphology. Proceedings of the National Academy of Sciences of the United States of America, 95(7): 36853689.

Noriega, J.A., C. Solis, F. Escobar y E. Realpe. 2007. Escarabajos coprófagos (Coleoptera: Scarabaeidae) de la provincia de la Sierra Nevada de Santa Marta. Biota Colombiana, 8(1): 77-86.

Noriega, J.A., W. Barranco, J. Hernández, E. Hernández, S. Castillo, D. Monroy y H. García. 2016. Estructura estacional del ensamblaje de escarabajos coprófagos (Coleoptera: Scarabaeinae) en una parcela permanente de bosque seco tropical. Revista de la Academia Colombiana de Ciencias Exactas, Físicas y Naturales, 40(154): 75-83.

Noriega, J.A., M. March-Salas, S. Castillo, H. García-Q, J. Hortal and A.M.C. Santos. 2021. Human perturbations reduce dung beetle diversity and dung removal ecosystem function. Biotropica, 53(3): 753-766.

Orozco, J. y M. Pérez. 2008. Escarabajos coprófagos (Coleoptera, Scarabaeoidea) del Parque Nacional Los Estoraques (Norte de Santander, Colombia). Revista Brasileira de Entomologia, 52(1): 36-40.

Ortega-Echeverría, C., G.R. Navas y J.A. Noriega. 2019. Estacionalidad del ensamblaje de escarabajos coprófagos (Coleoptera: Scarabaeinae) del jardín botánico de Cartagena "Guillermo Piñeres" Bolívar-Colombia. Caldasia, 41(1): 124-138.

Ortega-Martínez, I.J., C.E. Moreno and F. Escobar. 2016. A dirty job: manure remocal by dung beetles in both a cattle ranch and laboratory setting. Entomologia Experimentalis et Applicata, 161(1): 70-78.

Ortega-Martínez, I.J., C.E. Moreno, C.L. Rios-Díaz, L. Arellano, F. Rosas and I. Castellanos. 2020. Assembly mechanisms of dung beetles in temperate forests and grazing pastures. Scientific Reports, (10): 391.

Ortega-Martínez, I.J., C.E. Moreno, L. Arellano, I. Castellanos, F. Rosas and C.L. Ríos-Díaz. 2021. The relationship between dung beetle diversity and manure removal in forest and sheep grazed grasslands. Community Ecology.

Pablo-Cea, J.D., M.A. Velado-Cano and J.A. Noriega. 2020. A first step to evaluate the impact of ecotourism on biodiversity in El Salvador: a case study using dung beetles in a National Park. Journal of Ecotourism, 20(1): 51-69.
Padilla-Hill, D.N. and G. Halffter. 2007. Biogeography of the areas and Canthonini (Coleoptera: Scarabaeidae) of dry tropical forests in Mesoamerica and Colombia. Acta Zoológica Mexicana (n.s.), 23(1): 73-108.

Palacios-Ríos, M., V. Rico-Gray y E. Fuentes. 1990. Inventario preliminar de los Coleoptera Lamellicornia de la zona de Yaxchilan, Chiapas, México. Folia Entomológica Mexicana, (78): 49-60.

Paulian, R. 1933. Coprophages américains nouveaux ou peu connus (Col). Bulletin de la Société Entomologique de France, (38): 204-205.

Paulian, R. 1936. Sur quelques Onthophagus américains nouveaux ou peu connus (Col. Lamellicornes). Festschrift für 60. Geburtstage von Professor Dr. Embrik Strand, (1): 506-509.

Paulian, R. 1988. Biologie des coléoptères. Lechevalier, Paris.

Pereira, D. y B. Kohlmann. 2012. Estudio de biogeografía de islas en agroecosistemas de banano mediante el uso de escarabajos coprófagos como bioindicadores ecológicos. Tierra Tropical, 8(1): 95-113.

Pereira, F.S. y G. Halffter. 1961. Nuevos datos sobre Lamellicornia mexicanos con algunas observaciones sobre saprofagia. Revista Brasileira Entomologica, (10): 53-66.

Pérez-Cogollo, L.C., R.I. Rodríguez-Vivas, H. Delfín-González, E. Reyes-Novelo and M.A. Morón. 2015. Life history of Onthophagus landolti Harold, 1880 (Coleoptera: Scarabaeidae), with descriptions of the preimaginal stages. The Coleopterists Bulletin, 69(2): 255-263.

Pérez-Cogollo, L.C., R.I. Rodríguez-Vivas, G.S. Basto-Estrella, E. Reyes-Novelo, I. Martínez-Morales, M.M. Ojeda-Chi y M.E. Favila. 2018. Toxicidad y efectos adversos de las lactonas macrocíclicas sobre los escarabajos estercoleros: una revisión. Revista Mexicana de Biodiversidad, (89): 1293-1314.

Pérez-Villamares, J.C., E. Jiménez-Sánchez y J. Padi1la-Ramírez. 2016. Escarabajos atraídos a la carroña (Coleoptera: Scarabaeidae, Geotrupidae, Hybosoridae, Trogidae y Silphidae) en las cañadas de Coatepec Harinas, Estado de México, México. Revista Mexicana de Biodiversidad, 87: 443-450.

Pessôa, M.B., F. Alves-Martins, P. de Marco Júnior and J. Hortal. 2020. Unveiling the drivers of local dung beetle species richness in the Neotropics. Journal of Biogeography, 48(4): 861-871.

Peterson, A.T., A.G. Navarro-Siguenza, B.E. Hernández-Baños, G. Escalona-Segura, F. Rebón-Gallardo, Emir Rodríguez-Ayala, E.M. Figueroa-Esquivel and L. Cabrera-García. The Chimalapas Region, Oaxaca, Mexico: a high-priority region for bird Conservation in Mesoamerica. Bird Conservation International, (13): 227-253.

Philips, T.K. 2011. 2. The evolutionary history and diversification of dung beetles. (pp. 21-46). En: Simmons, L.W. y T.J. Ridsdill-Smith (Eds.). Ecology and evolution of dung beetles. Blackwell Publishng Ltd, Chichester.

Philips, T.K. 2016. Phylogeny of the Oniticellini and On- 
thophagini dung beetles (Scarabaeidae, Scarabaeinae) from morphological evidence. ZooKeys, (579): 9-57.

Pineda, E., C. Moreno, F. Escobar and G. Halffter. 2005. Frog, bat, and dung beetle diversity in the cloud forest and coffee agoecosystems of Veracruz, Mexico. Conservation Biology, 19 (2): 400-410.

Pinilla-Buitrago, G.E., T. Escalante, A. Gutiérrez-Velázquez, P. Reyes-Castillo and O.R. Rojas-Soto. 2018. Areas of endemism persist through time: A palaeoclimatic analysis in the Mexican Transition Zone. Journal of Biogeography, 45(4): 952-961.

Pulido-Herrera, L.A. y M. Zunino. 2007. Catálogo preliminar de los Onthophagini de América (Coleoptera: Scarabaeine). (pp. 93-129). En: Zunino, M. y A. Melic (Eds.). Escarabajos, diversidad y conservación biológica. Ensayos en homenaje a Gonzalo Halffter. Sociedad Entomológica Aragonesa, Monografías 3ercer Milenio M3M, Zaragoza.

Quiroz-Rocha, G.A., J.L. Navarrete-Heredia y P.A. Martínez-Rodríguez. 2008. Especies de Scarabaeinae (Coleoptera: Scarabaeidae) y Silphidae (Coleoptera) necrófilas de Bosque de Pino-Encino y Bosque Mesófilo de Montaña en el Municipio de Mascota, Jalisco, México. Dugesiana, 15(1): 27-37.

Ramírez-Ponce, A., J Allende-Canseco y M.A. Morón. 2009. Fauna de coleópteros lamelicornios de Santiago Xiacui, Sierra Norte, Oaxaca, México. Acta Zoológica Mexicana (n.s.), 25(2): 323-343.

Ramírez-Ponce, A., J.M. Calderón-Patrón, H.M. Guzmán-Vásquez and C.E. Moreno. 2019. Biotic heterogeneity among scarab beetle communities in an anthropized landscape in the Central Valleys of Oaxaca, Mexico. Journal of Insect Conservation, (23): 765-776.

Ramírez-Restrepo, L. and G. Halffter. 2016. Copro-necrophagous beetles (Coleoptera: Scarabaeinae) in urban areas: A global review. Urban Ecosystems, (19): 11791195.

Rangel-Acosta, J.L. y N.J. Martínez-Hernández. 2017. Comparación de los ensamblajes de escarabajos copronecrófagos (Scarabaeidae: Scarabaeinae) entre fragmentos de bosque seco tropical y la matriz adyacente en el departamento del Atlántico-Colombia. Revista Mexicana de Biodiversidad, (88): 389-401.

Rangel-Acosta, J.L., N.J. Martínez-Hernández, B.P. Gutierrez-Rapalino, L.C. Gutierrez-Moreno y R.A. Borja-Acuña. 2016. Efecto del tamaño de la ronda hidráulica sobre las comunidades de escarabajos coprófagos (Scarabaeidae: Scarabaeinae) en la cuenca media y baja del rio Cesar, Colombia. Entomotrópica, 31(15): 109130.

Rangel-Acosta, J.L., J.A. Solano-Torres, N.J. Martínez-Hernández. 2018. Variación temporal y vertical de los escarabajos coprófagos (Scarabaeidae: Scarabaeinae) en dos fragmentos de bosque tropical en el departamento del Atlántico-Colombia. Boletín Científico Centro de Museos, Museo de Historia Natural, 22(1): 179-198.

Rangel-Acosta, J.L., N.J. Martínez-Hernández y R. Yonoff-Zapata. 2020. Respuesta de los escarabajos coprófagos (Scarabaeidae: Scarabaeinae) a la modificación del hábitat causada por un incendio forestal en la Reserva Bijibana, Atlántico-Colombia. Revista Mexicana de Biodiversidad, (91): e912879

Ratcliffe, B.C., M.L. Jameson, L. Figueroa, R.D. Cave, M.J. Paulsen, E.B. Cano, C. Beza-Beza, L. Jiménez-Ferbans and P. Reyes-Castillo. 2015. Beetles (Coleoptera) of Peru: A Survey of the Families. Scarabaeoidea. Journal of the Kansas Entomological Society, 88(2): 186-207.

Reddell, J.R. 1981. A review of the cavernicole fauna of Mexico, Guatemala, and Belize. Bulletin of the Texas Memorial Museum, the University of Texas at Austin, (27): 1-327.

Reyes-Novelo, E., H. Delfín-González and M.A. Morón. 2007. Copro-necrophagous beetle (Coleoptera: Scarabaeidae) diversity in an agroecosystem in Yucatan, Mexico. Revista de Biología Tropical, 55(1): 83-99.

Rivera, J.D., B. Gómez, D.A. Navarrete-Gutiérrez, L. Ruíz-Montoya, L. Delgado and M.E. Favila. 2020. Mechanisms of diversity maintenance in dung beetle assemblages in a heterogeneous tropical landscape. PeerJ, (8): e9860.

Rivera-Gasperín, S.L. y F. Escobar-Hernández. 2020. Especies de Scarabaeoidea (Coleoptera) del CICOLMA, Veracruz, México. Acta Zoológica Mexicana (n.s.), (36): $1-19$.

Rivera-Gasperín, F. Escobar-Hernández and M.E. Favila. 2021. An update on the feeding preferences of the dung beetle Onthophagus moroni Zunino and Halffter (Coleoptera: Scarabaeidae) from the Cuetzalan Caves, Puebla, Mexico. The Coleopterists Bulletin, 75(1): 247255.

Robinson, M. 1940. Studies in the Scarabaeidae, II (Coleoptera). Transactions of the American Entomological Society, (66): 141-159.

Robinson, M. 1948. Remarks on a few Scarabaeidae (Coleoptera). Entomological News, (59): 175-177.

Rodríguez, W.D., J.L. Navarrete-Heredia, M. Vásquez-Bolaños, R. Rodríguez-Macias y G.A. Briceño-Félix. 2019a. Escarabajos asociados a Agave tequilana Weber variedad azul. Universidad de Guadalajara, Jalisco.

Rodríguez, W.D., J.L. Navarrete-Heredia, R. Rodríguez-Macías, G.A. Briceño-Félix, M. Vásquez-Bolaños and J. Klimaszewski. 2019. Diversity patterns of necrocolous beetles (Coleoptera: Scarabaeidae, Silphidae, Trogidae) in Agave tequilana Weber (Asparagaceae) fields of different ages. The Canadian Entomologist, 151(5): 585-599.

Rodríguez-López, M.E. G. Sánchez-Hernández y B. Gómez. 2019. Escarabajos coprófagos (Coleoptera: Scarabaeidae: Scarabaeinae) en la reserva El Zapotal, Chiapas, México. Revista Peruana de Biología, 26(3): 339-350.

Rodríguez-Vivas, R.I., G.S. Basto-Estrella, E. Reyes-Novelo, L.C. Pérez-Cogollo, W. Arcila-Fuentes, M. Ojeda-Chi and I. Martínez-M. 2019. Onthophagus landolti and Canthon indigaceus chevrolati (Coleoptera: Scarabaeinae) are attracted to the feces of ivermectin-treated cattle in the Mexican tropics. Revista de Biología Tropical, 67(1): 254-265. 
Rös, M., F. Escobar and G. Halffter. 2012. How dung beetles respond to a human-modified variegated landscape in Mexican cloud forest: a study of Biodiversity integrating ecological and biogeographical perspectives. Diversity and Distributions, (18): 377-389.

Rossini, M., F.Z. Vaz-de-Mello and M. Zunino. 2018a. Toward a comprehensive taxonomic revision of the "hirculus" group of American Onthophagus Latreille, 1802 (Coleoptera, Scarabaeidae, Scarabaeinae). European Journal of Taxonomy, (432): 1-21.

Rossini, M., F.Z. Vaz-de-Mello and M. Zunino. 2018b. A taxonomic revision of the New World Onthophagus Latreille, 1802 (Coleoptera: Scarabaeidae: Scarabaeinae) of the osculatii species-complex, with description of two new species from South America. Journal of Natural History, 52 (9-10): 541-586.

Ruíz-Pérez, I., J.L. León-Cortés, L. Arellano y D. Navarrete. 2019. Manejo forestal comunitario en el sur de México: ¿es una práctica sustentable para el mantenimiento de los ensambles de escarabajos? Revista Mexicana de Biodiversidad, (90): e902564.

Rzedowski, J. 2006. Vegetación de México. Primera edición digital. Comisión Nacional para el Conocimiento y Uso de la Biodiversidad, Ciudad de México.

Salomão, R.P., D. González-Tokman, W. Dáttilo, J.C. López-Acosta and M.E. Favila. 2018. Landscape structure and composition define the body condition of dung beetles (Coleoptera: Scarabaeinae) in a fragmented tropical rainforest. Ecological Indicators, (88) 144-151.

Salomão, R.P., M.E. Favila and D. González-Tokman. 2020. Spatial and temporal changes in the dung beetle diversity of a protected, but fragmented, landscape of the northernmost Neotropical rainforest. Ecological Indicators, (111): 105968.

Salomão, R.P., A. Arriaga-Jiménez and B. Kohlmann. 2021. The relationship between altitudinal gradients, diversity, and body size in a dung beetle (Coleoptera: Scarabaeinae: Onthophagus) model system. Canadian Journal of Zoology, (99): 33-43.

Sánchez-de-Jesús, H.A., V. Arroyo-Rodríguez, E. Andresen and F. Escobar. 2016. Forest loss and matrix composition are the major drivers shaping dung beetle assemblages in a fragmented rainforest Landscape Ecology, (31): 843-854.

Sánchez-Hernández, G. and B. Gómez. 2018. First precise locality data for Onthophagus atriglabrus Howden and Gill and new state record for Onthophagus anewtoni Howden and Génier (Coleoptera: Scarabaeidae: Scarabaeinae) in Mexico. The Coleopterists Bulletin, 72(4): 873-876.

Sánchez-Hernández, G., B. Gómez y Gómez, L. Delgado y M. E. Rodríguez-López. 2017. Primer registro de Onthophagus longimanus Bates, 1887 (Coleoptera: Scarabaeidae: Scarabaeinae) para Chiapas, México. Dugesia$n a, 24(1): 57-59$.

Sánchez-Hernández, G., B. Gómez, L. Delgado, M.E. Rodríguez-López y E.R. Chamé-Vázquez. 2018. Diversidad de escarabajos copronecrófagos (Coleoptera: Scarabaeidae: Scarabaeinae) en la Reserva de la Biosfera
Selva El Ocote, Chiapas, México. Caldasia, 40(1): 144160.

Sánchez-Hernández, G., B. Gómez, E.R. Chamé-Vázquez, R.A. Dávila-Sánchez, M.E. Rodríguez-López and L. Delgado. 2020. Current status of dung beetles (Coleoptera, Scarabaeidae, Scarabaeinae) diversity and conservation in Natural Protected Areas in Chiapas (Mexico). Neotropical Biology and Conservation, 15(3): 219-244. Sánchez-Huerta, J.L., M. Tonelli, M. Zunino and G. Halffter. 2015. Redescription of Onthophagus halffteri Zunino (Coleoptera: Scarabaeidae: Scarabaeinae), with ecological and distributional notes. The Coleopterists Bulletin, 69(2): 225-230.

Sánchez-Huerta, J.L., M. Zunino and G. Halffter. 2018. A new species of American Onthophagus Latreille (Coleoptera: Scarabaeidae: Scarabaeinae) associated with rodent (Geomyidae) burrows. The Coleopterists Bulletin, 72(3): 407-416.

Sánchez-Huerta, J.L., V. Moctezuma y G. Halffter. 2019. Nuevo registro de distribución de Canthon angustatus Harold en Veracruz, México. Southwestern Entomologist, 44(1): 353-355.

Sanmartin, I., H. Enghoff and F. Ronquist. 2001. Patterns of animal dispersal, vicariance and diversification in the Holarctic. Biological Journal of the Linnean Society, (73): 345-390.

Sansom, R. 2003. Constraining the adaptationism debate. Biology and Philosophy, (18): 493-512.

Santiago-Molina, J.P., I.A. Chamorro-Florescano, S.J. Amésquita-Melo y J.M. Pech-Canché. 2014. Escarabajos copro-necrófagos (Coleoptera: Scarabaeinae) en fragmentos de Selva Baja Caducifolia, Pastizales y Cultivos de Vainilla en San Lorenzo Tajín, Papantla, Veracruz, México. Revista Científica Biológico Agropecuaria Tuxpan, 2(4): 842-852.

Santos-Heredia, C., E. Andresen, D.A. Zárate and F. Escobar. 2019. Dung beetles and their ecological functions in three agroforestry systems in the Lacandona rainforest of Mexico. Biodiversity and Conservation, (27): 2379-2394.

Santos-Moreno, A. 2014. Los mamíferos del estado de Oaxaca. Revista Mexicana de Mastozoología Nueva Época, 4(2): 18-32.

Sarges, R., G. Halffter and A. Díaz-Rojas. 2012. The importance of frugovory to the survival of the dung beetle Onthophagus rhinolophus Harold (Coleoptera: Scarabaeidae: Scarabaeinae) under changing ecological conditions. The Coleopterists Bulletin, 66(2): 166-168.

Say, T. 1835. Descriptions of new north American coleopterous Insects, and observations on some already described. Boston Journal of Natural History, (1): 151-203.

Schaeffer, C.F. 1909. New Coleoptera chiefly from Arizona. The Museum of the Brooklyn Institute of Arts and Sciences, Science Bulletin, 1(15): 375-386.

Schaeffer, C.F. 1914. A short review of the North American species of Onthophagus (Col.: Scarab.). Journal New York Entomological Society, (22): 290-300.

Schoolmeesters, P. 2021. Scarabs: World Scarabaeidae Database (version 2021-03-29). https://www.catalo- 
gueoflife.org/data/dataset/1027. Fecha de consulta: 01 de mayo de 2021.

Schwery, O and B.C. O'Meara. 2021. Age, origin, and biogeography: Unveiling the factors behind the diversification of dung beetles. bioRxiv.

Servín-Pastor, M., R.P. Salomão, F. Caselín-Cuevas, A. Córdoba-Aguilar, M.E. Favila, A. Jácome-Hernández, D. Lozano-Sánchez and D. González-Tokman. 2020. Malnutrition and parasitism shape ecosystem services provided by dung beetles. Ecological Indicators, (121): 107205.

Slay, M.E., P.E. Skelley and S.J. Taylor. 2012. New records of Onthophagus cavernicollis Howden and Cartwright (Coleoptera: Scarabaeidae) from Ozark Caves, with a review of scarabaeoids reported from North American Caves. The Coleopterists Bulletin, 66(3): 187-199.

Solís, C., J.A. Noriega y G. Herrera. 2011. Escarabajos coprófagos (Coleoptera: Scarabaeinae) en tres bosques secos del departamento del Atlántico-Colombia. Boletín del Museo de Entomología de la Universidad del Valle, 12(1): 33-41.

Solís, Á. and B. Kohlmann. 2012. Checklist and distribution atlas of the Scarabaeinae (Coleoptera: Scarabaeidae) of Costa Rica. Zootaxa, (3482): 1-32.

Solórzano-Kraemer, M.M. 2007. Systematic, palaeoecology, and palaeobiogeography of the insect fauna from Mexican amber. Palaeontographica Abteilung A, 282(1-6): 1-133.

Tarasov, S.I. and A.Y. Solodovnikov. 2011. Phylogenetic analyses reveal reliable morphological markers to classify mega-diversity in Onthophagini dung beetles (Coleoptera: Scarabaeidae: Scarabaeinae). Cladistics, (27): $1-39$.

Tarasov, S. and D. Dimitrov. 2016. Multigene phylogenetic analysis redefines dung beetles relationships and classification (Coleoptera: Scarabaeidae: Scarabaeinae). BMC Evolutionary Biology, (16): 257.

Tello, F., J.R. Verdú, M. Rossini and M. Zunino. 2021. Onthophagus pilauco sp. nov. (Coleoptera, Scarabaeidae): evidence of beetle extinction in the Pleistocene-Holocene transition in Chilean Northern Patagonia. ZooKeys, (1043): 133-145.

Thomas, D.B. 1993. Scarabaeidae (Coleoptera) of the Chiapanecan forests: A faunal survey and chorographic analysis. The Coleopterists Bulletin, 47(4): 363-408.

Tovar, H.L., J.A. Noriega y P. Caraballo. 2016. Efecto de la ivermectina sobre la estructura del ensamble de escarabajos coprófagos (Coleoptera: Scarabaeidae: Aphodiinae-Scarabaeinae) en las sabanas colombianas de la región Caribe. Actualidades Biológicas, 38(105): 157166.

Trevilla-Rebollar, A., C. Deloya y J. Padilla-Ramírez. 2010. Coleópteros necrófilos (Scarabaeidae, Silphidae y Trogidae) de Malinalco, Estado de México, México. Neotropical Entomology, 39(4): 486-495.

Trujillo-Miranda, A.L., H. Carrillo-Ruíz, S.P. Rivas-Arancibia y A.R. Andrés-Hernández. 2016. Estructura y composición de la comunidad de escarabajos (Coleoptera: Scarabaeoidea) en el cerro Chacateca, Zapotitlán,
Puebla, México. Revista Mexicana de Biodiversidad, 87: 109-122.

Vaz-de-Mello, F.Z. 2000. Estado atual do conhecimento dos Scarabaeidae s. str. (Coleoptera: Scarabaeoidea) do Brasil. (pp. 183-195). En: Martín-Piera, F., J.J. Morrone y A. Melic (Eds.). Hacia un proyecto CYTED para el inventario y estimación de la diversidad entomológica en Iberoamérica: PrIBES 2000. Monografías 3ercer Milenio M3M, Sociedad Entomológica Aragonesa, Zaragoza.

Vaz-de-Mello, F.Z., J. Louzada and J.H. Schoereder. 1998. New data and comments on Scarabaeidae (Coleoptera: Scarabaeoidea) associated with Attini (Hymenoptera: Formicidae). The Coleopterists Bulletin, 52(3): 209216.

Velázquez-López, L.F., B. Gómez y Gómez, M. Rincón-Rabanales y E. Chame-Vázquez.2019. Diversidad de escarabajos copronecrófagos (Coleoptera: Scarabaeidae: Scarabaeinae) en el sistema costero de Puerto Madero (Chiapas, México). Boletín de la Sociedad Entomológica Aragonesa, (64): 186-192.

Verdú, J.R., C.E. Moreno, G. Sánchez-Rojas, C. Numa, E. Galante and G. Halffter. 2007. Grazing promotes dung beetle diversity in the xeric Landscape of a Mexican Biosphere Reserve. Biological Conservacion, (140): 308-317.

Villamarín-Cortez, S. 2010. Escarabajos estercoleros (Coleoptera: Scarabaeidae) de El Goaltal, provincia de Carchi, Ecuador: lista anotada de especies y ecología. Avances en Ciencias e Ingenierías, Sección B, 2(3): 98-103.

Villaseñor, J.L. y E. Ortiz. 2014. Biodiversidad de las plantas con flores (División Magnoliophyta) en México. Revista Mexicana de Biodiversidad, Suplemento, (85): 134-142.

Vulcano, M.A. e F.S. Pereira. 1967. Sinópse dos Passalidae e Scarabaeidae s. str. da região amazónica (Insecta, Coleoptera). Atas do Simposio sôbre a Biota Amazônica (Zoologia), (5): 533-603.

Werner, M. and L.W. Simmons. 2008. The evolution of male genitalia: functional integration of genital sclerites in the dung beetle Onthophagus taurus. Biological Journal of the Linnean Society, (93): 257-266.

Whitworth, A., C. Beirne, E. Flatt, G. Froese, C. Nuñez and A. Forsyth. 2021. Recovery of dung beetle biodiversity and traits in a regenerating rainforest: a case study from Costa Rica's Osa Peninsula. Insect Conservation and Diversity.

Wolfe, J.A. 1978. A paleobotanical interpretation of Tertiary climates in the Northern Hemisphere. American Scientist, (66): 694-703.

Yanes-Gómez, G. y M.A. Morón. 2010. Fauna de coleópteros Scarabaeoidea de Santo Domingo Huehuetlán, Puebla, México. Su potencial como indicadores ecológicos. Acta Zoológica Mexicana (n.s.), 26(1): 123-145.

Yanes-Gómez, G., M. Pérez-Méndez, O.I. Ramírez-González, M.A. Morón, H. Carrillo-Ruíz y A.A. Romero-López. 2015. Diversidad de coleópteros copro-necrófagos en el "Rancho Canaletas", Paso del Macho Veracruz, México. Acta Zoológica Mexicana (n.s.), 
31(2): 283-290.

Young, O. 1981. The attraction of the neotropical Scarabaeinae (Coleoptera: Scarabaeidae) to reptile and amphibian fecal material. The Coleopterists Bulletin, 35(3): 345-348.

Young, O.P. 2015. Predation on dung beetles (Coleoptera: Scarabaeidae): a literature review. Transactions American Entomological Society, (141): 111-155.

Zamora-Vuelvas, M.C., J. Ponce-Saavedra y A.C. Deloya-López. 2014. Scarabaeoidea (Insecta: Coleoptera) capturados con trampas en el Cerro "El Águila", municipio de Morelia, Michoacán. Entomología Mexicana, (1): 519-524.

Zaragoza-Caballero, S. and C.X. Pérez-Hernández. 2017. An annotated catalogue of the Coleoptera types deposited in the National Insect Collection (CNIN) of the National Autonomous University of Mexico. Zootaxa, 4288(1): 1-128.

Zaragoza-Caballero, S., G.M. Rodríguez-Mirón, V. Vega-Badillo, M. González-Ramírez, M.L. Zurita-García, D.E. Domínguez-León, S. López-Pérez, I.G. Gutiérrez-Carranza, P. Cifuentes-Ruiz, C.X. Pérez-Hernández, E. Ramírez del Valle and N. Gutiérrez. 2019. A checklist of the Coleoptera (Insecta) from Morelos, Mexico. Zootaxa, 4580(1): 1-122.

Zunino, M. 1980. Note sul brachitterismo nel genere Onthophagus Latr. (Coleoptera, Scarabaeoidea). Bollettino del Museo di Zoologia dell'Università di Torino, (2): 9-12.

Zunino, M. 1981. Note sul alcuni Onthophagus americani e descrizione di nuove specie (Coleoptera: Scarabaeidae).

Recibido: 12 mayo 2021

Aceptado: 17 junio 2021

Cuadro 1. Riqueza de especies de Onthophagus en diversos países americanos.

\begin{tabular}{|c|c|c|}
\hline País & $\begin{array}{l}\text { Riqueza } \\
\text { de espe- } \\
\text { cies }\end{array}$ & Fuente \\
\hline Canadá & 4 & Howden y Cartwright 1963 \\
\hline Estados Unidos & 33 & $\begin{array}{l}\text { Howden y Cartwright } \\
\text { 1963; Howden } 1973\end{array}$ \\
\hline México & 120 & Presente estudio \\
\hline Nicaragua & 13 & Maes 1998 \\
\hline Costa Rica & 41 & $\begin{array}{l}\text { Kohlmann et al. 2019; pre- } \\
\text { sente estudio. }\end{array}$ \\
\hline Panamá & 26 & Delgado y Curoe 2014 \\
\hline Colombia & 26 & Escobar 2000 \\
\hline Ecuador & 24 & $\begin{array}{l}\text { Chamorro et al. 2019; } \\
\text { Moctezuma y Halffter } \\
\text { 2020c }\end{array}$ \\
\hline Perú & 8 & Ratcliffe et al. 2015 \\
\hline Brasil & 19 & Vaz-de-Mello 2000 \\
\hline
\end{tabular}

Bollettino del Museo di Zoologia dell'Università di Torino, (6): 75-86.

Zunino, M. 2003. Tribu Onthophagini. (pp. 66-74). En: Morón, M.A. (Ed.). Atlas de los escarabajos de México. Coleoptera: Lamellicornia. Vol. II. Familias Scarabaeidae, Trogidae, Passalidae y Lucanidae. Argania editio, Barcelona.

Zunino, M. y G. Halffter. 1988a. Análisis taxonómico, ecológico y biogeográfico de un grupo americano de $\mathrm{On}$ thophagus (Coleoptera: Scarabaeidae). Museo Regionale di Scienze Naturali, Monografie, (9): 1-211.

Zunino, M. y G. Halffter. 1988b. Nueva especie de Onthophagus (Coleoptera, Scarabaeidae) asociada a cuevas. Folia Entomológica Mexicana, (75): 17-32.

Zunino, M. y G. Halffter. 1988c. Una nueva especie braquíptera de Onthophagus de México (Coleoptera, Scarabaeidae). Elytron, (2): 137-142.

Zunino, M. y G. Halffter. 1997. Sobre Onthophagus Latreille, 1802 americanos (Coleoptera: Scarabaeidae: Scarabaeinae). Elytron, (11): 157-178.

Zunino, M. y G. Halffter. 2005. Onthophagus fuscus parafuscus Zunino y Halffter, nuevo nombre para $O$. fuscus orientalis Zunino y Halffter, 1988. Acta Zoológica Mexicana (n.s.), 21(3): 149.

Zunino, M. and G. Halffter. 2007. The association of Onthophagus Latreille, 1802 beetles (Coleoptera: Scarabaeinae) with vertebrate burrows and caves. Elytron, (21): 17-55.

Cuadro 2. Riqueza de especies por patrón de distribución de las especies mexicanas de Onthophagus.

\begin{tabular}{ll}
\hline Patrón de distribución & $\begin{array}{l}\text { Riqueza de es- } \\
\text { pecies }\end{array}$ \\
\hline Paleoamericano de montaña & 57 \\
Paleoamericano tropical & 39 \\
Paleoamericano del Altiplano Mexicano & 14 \\
Paleoamericano mesoamericano & 7 \\
Paleoamericano bajacaliforniano & 1 \\
Desconocido & 2 \\
\hline
\end{tabular}




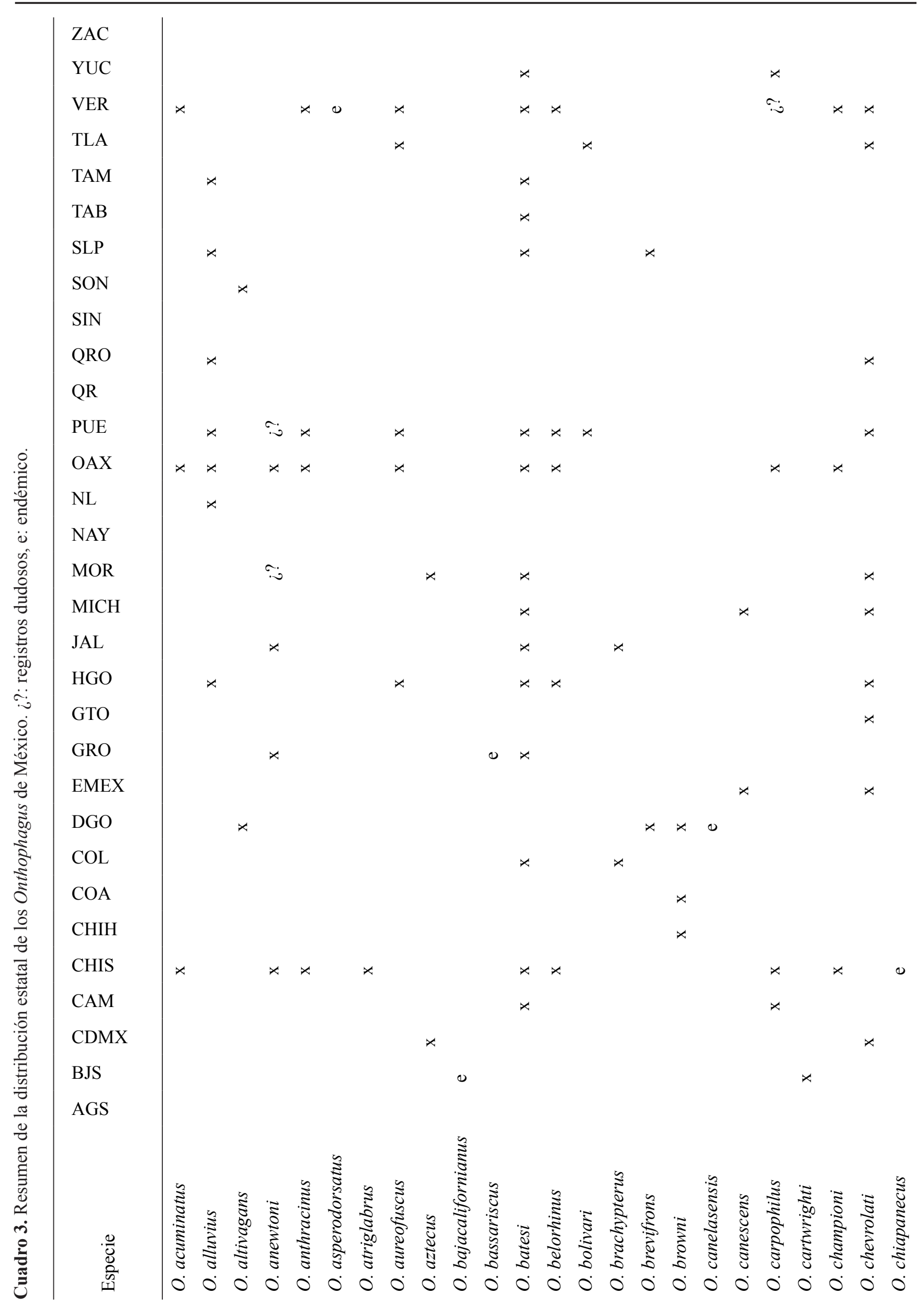


El género Onthophagus Latreille, 1802 (Coleoptera: Scarabaeidae) de México

ZAC

YUC

VER

TLA

TAM

TAB

SLP

SON

SIN

QRO

QR

PUE

OAX

NL

NAY

MOR

$\mathrm{MICH}$

JAL

HGO

GTO

GRO

EMEX

DGO

$\mathrm{COL}$

$\mathrm{COA}$

$\mathrm{CHIH}$

CHIS

CAM

CDMX

BJS

AGS

0
0
0
क्ञ

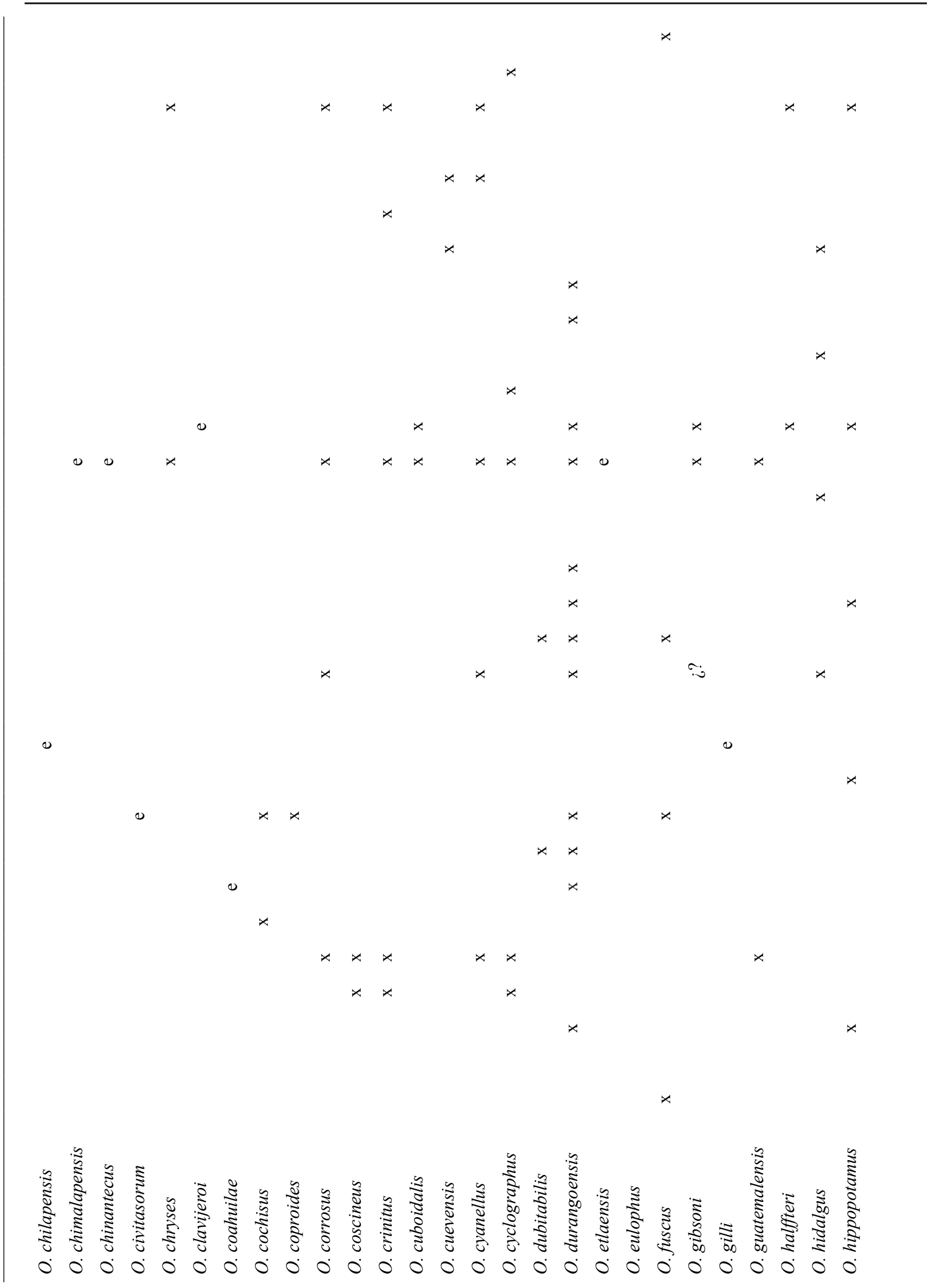

(C) 2021 Universidad de Guadalajara, Dugesiana 28(2): 175-220 


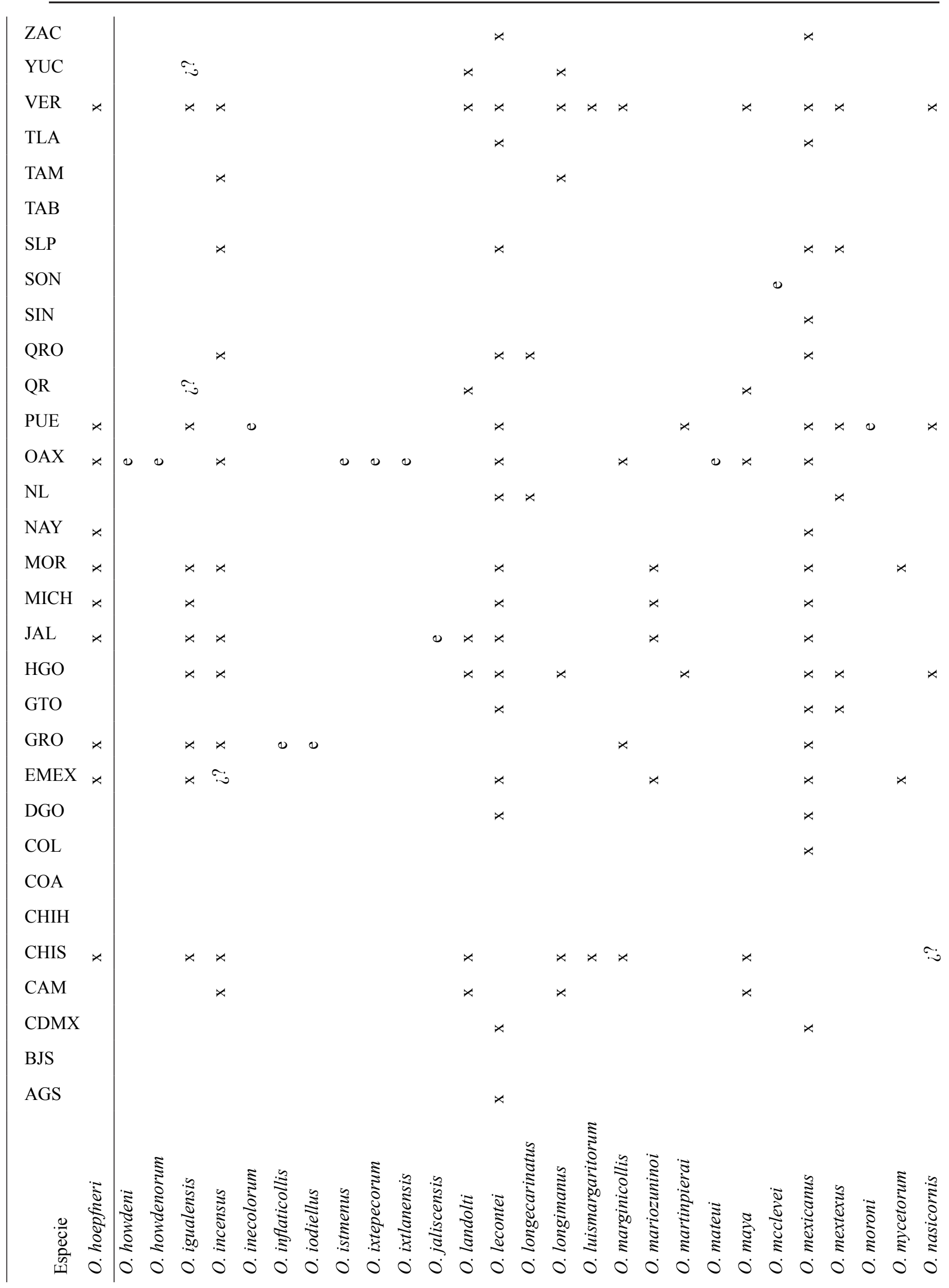


El género Onthophagus Latreille, 1802 (Coleoptera: Scarabaeidae) de México

\section{$\mathrm{ZAC}$}

YUC

VER

TLA

TAM

TAB

SLP

SON

SIN

QRO

QR

PUE

OAX

NL

NAY

MOR

$\mathrm{MICH}$

JAL

HGO

GTO

GRO

EMEX

DGO

$\mathrm{COL}$

$\mathrm{COA}$

$\mathrm{CHIH}$

CHIS

CAM

CDMX

BJS

AGS

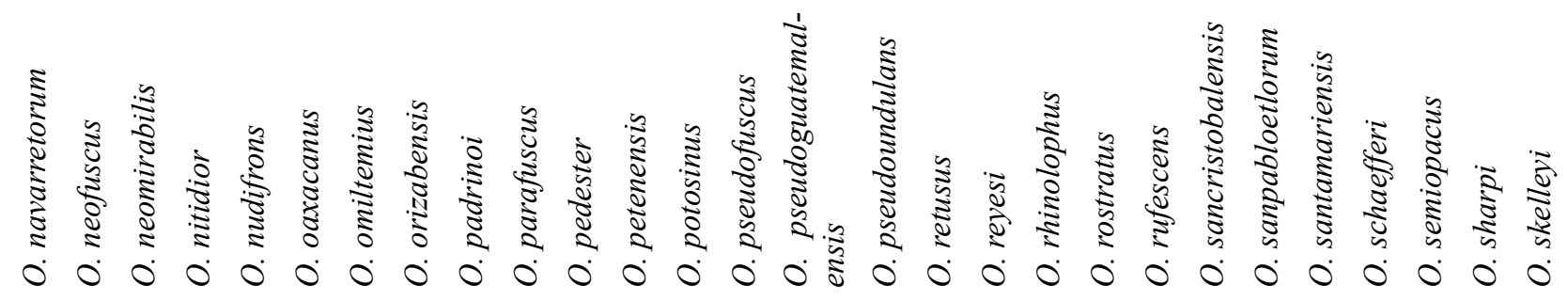




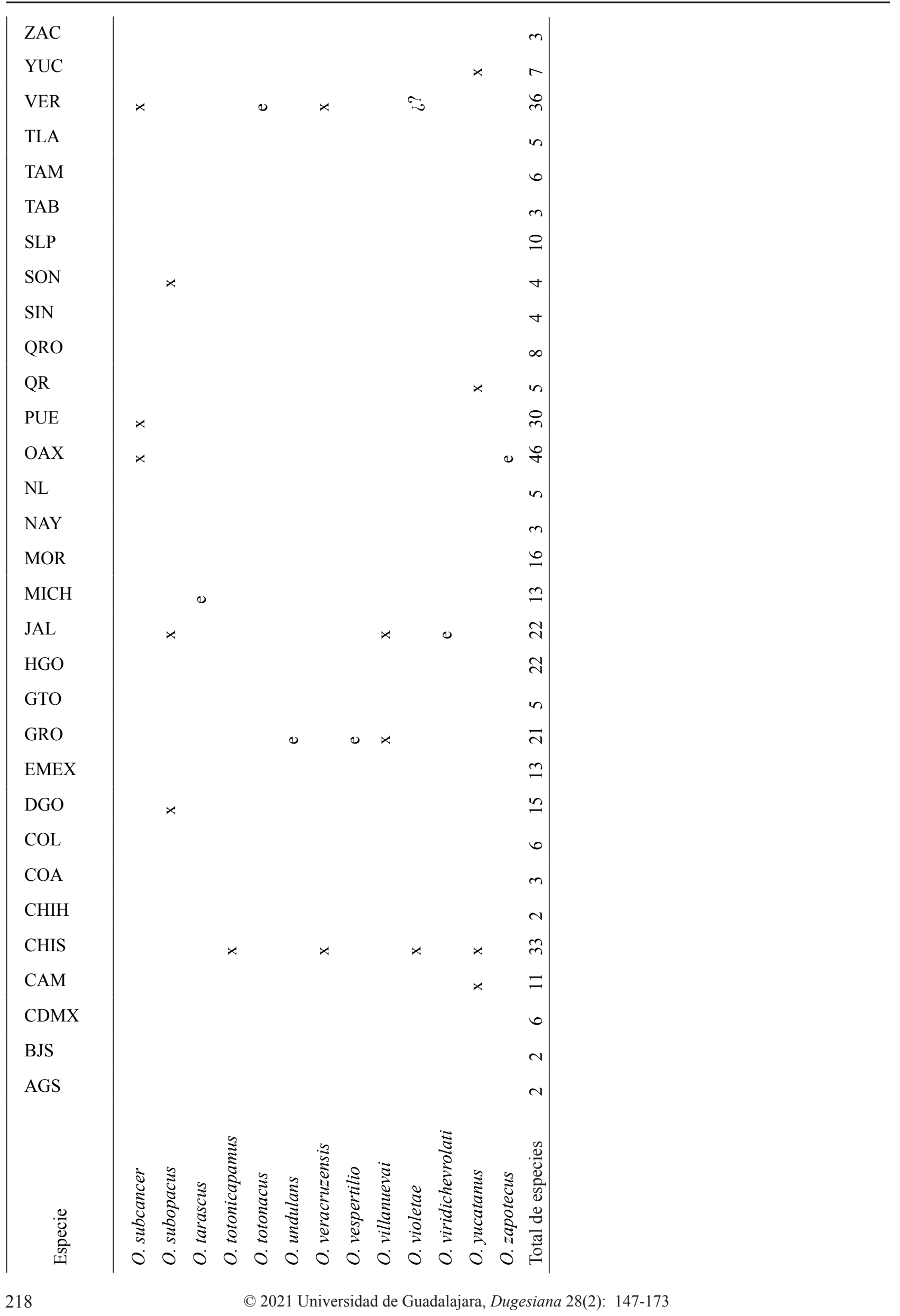




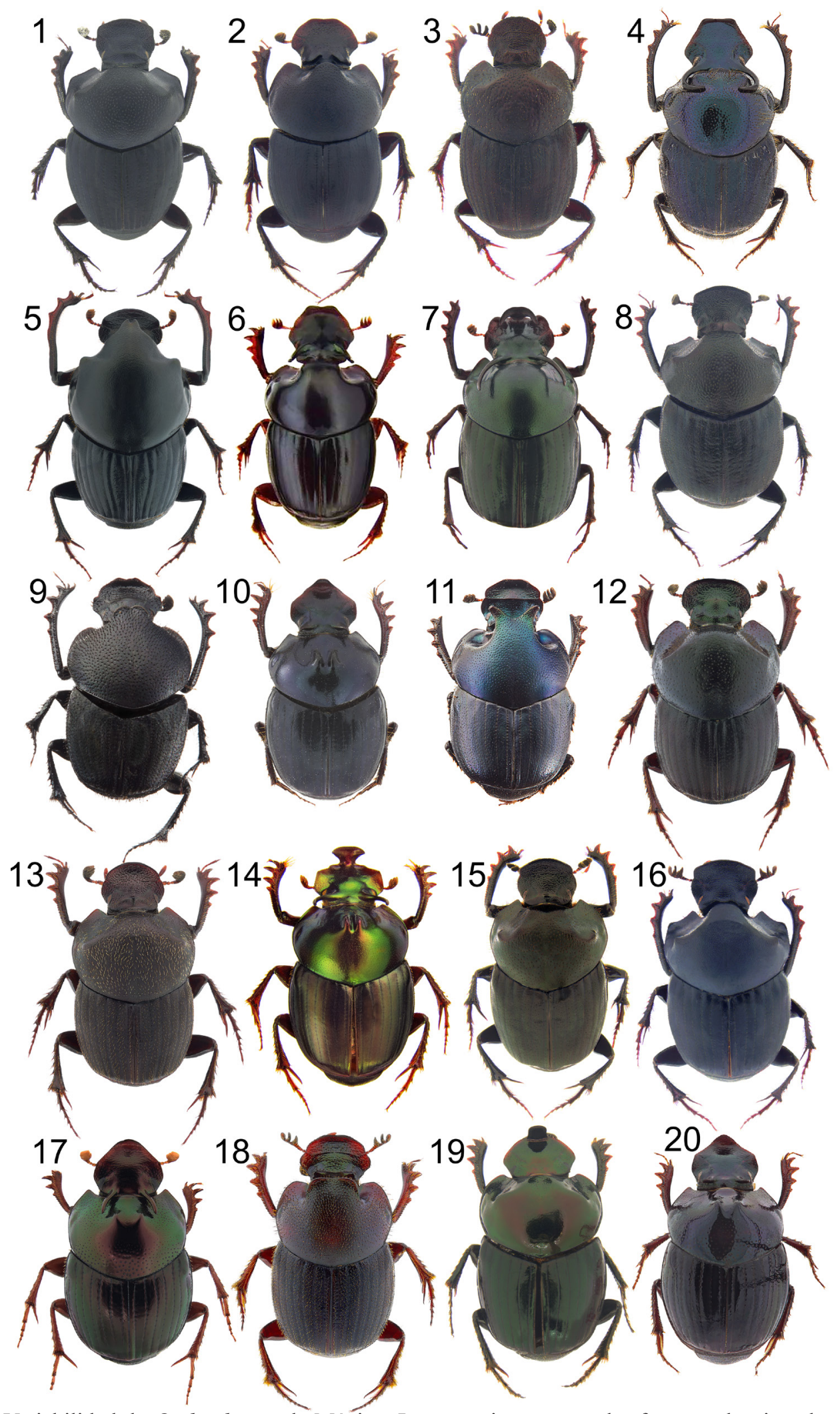

Figuras 1-20. Variabilidad de Onthophagus de México. Las especies presentadas fueron seleccionadas de acuerdo con su disponibilidad, y sus imágenes siguen un orden alfabético de forma descendente y de derecha a izquierda: 1. $O$. $b a$ jacalifornianus. 2. $O$. cartwrighti. 3. $O$. civitasorum. 4. $O$. crinitus. 5. $O$. halffteri. 6. $O$. incensus. 7. $O$. istmenus. 8. $O$. inecolorum. 9. O. lecontei. 10. O. maya. 11. O. mexicanus. 12. O. moroni. 13. O. pseudofuscus. 14. O. rhinolophus. 15. O. retusus. 16. O. sancristobalensis. 17. O. santamariensis. 18. O. totonacus. 19. O. villanuevai. 20. O. yucatanus. 4, 10 y 20 fueron modificados de Capello y Halffter (2019). 


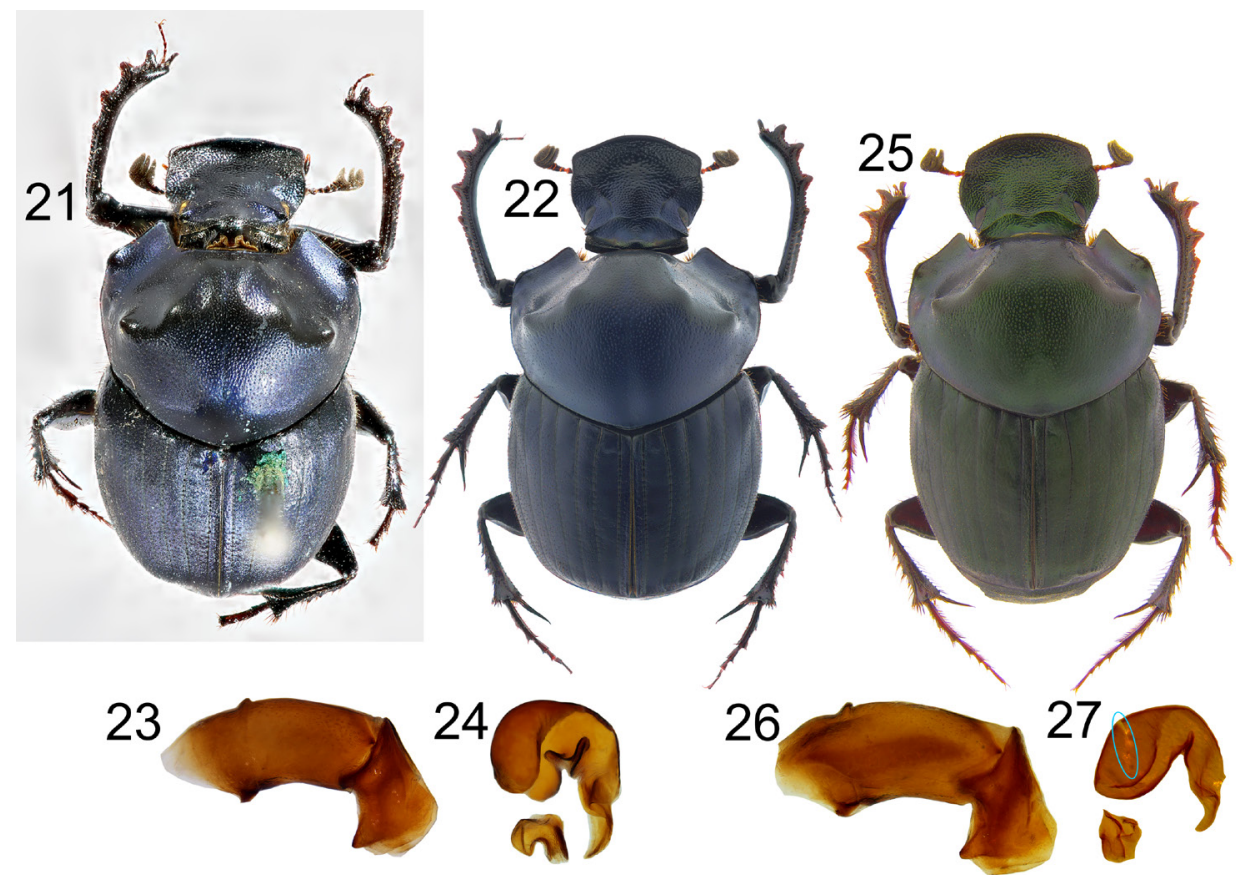

Figuras 21-27. O. cyanellus: 21. Lectotipo macho major (Volcán de Chiriquí, Panamá). 22. Hábito dorsal de un macho major (Sierra de Talamanca, Costa Rica). 23. Edeago (Sierra de Talamanca, Costa Rica). 24. Endofalito copulador (arriba) y endofalito adicional medio (abajo) (Sierra de Talamanca, Costa Rica). O. mesoamericanus (Cedros, San José, Costa Rica): 25. Hábito dorsal del holotipo macho major. 26. Edeago. 27. Endofalito copulador (arriba) y endofalito adicional medio (abajo), la elipse azul indica el área del lóbulo derecho que fue dañada durante la disección y preparación original del endofalito copulador. El daño en esta estructura fue previamente reportado por Halffter et al. (2019).

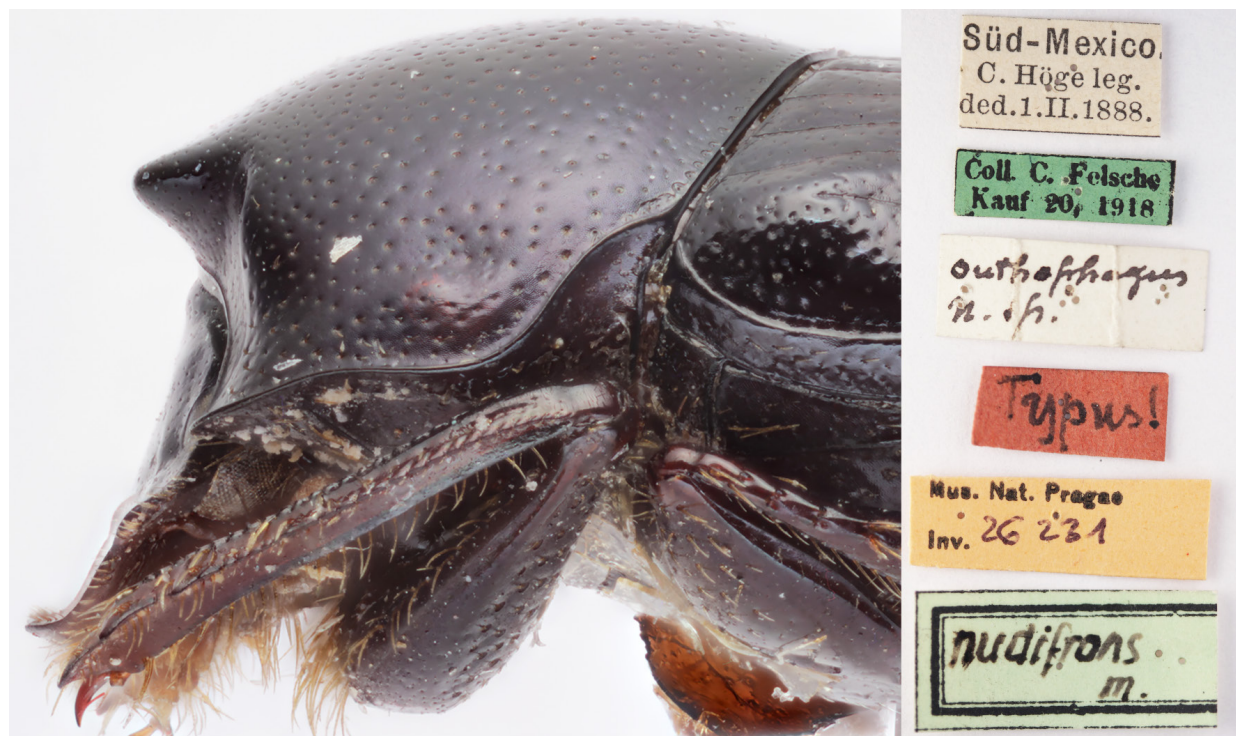

Figura 28. Holotipo de $O$. nudifrons, actualmente depositado en el Národní Muzeum de Praga, República Checa (cortesía de Jiř́i Hájek). 